\title{
Long Term Application of a Vehicle-Based Health Monitoring System to Short and Medium Span Bridges and Damage Detection Sensitivity
}

\author{
Ayaho Miyamoto', Jari Puttonen'1, Akito Yabe ${ }^{2}$ \\ ${ }^{1}$ Department of Civil Engineering, Aalto University, Aalto, Finland \\ ${ }^{2}$ Sustainable Solutions Department, KOZO KEIKAKU Engineering Inc., Tokyo, Japan \\ Email: miya818@yamaguchi-u.ac.jp
}

How to cite this paper: Miyamoto, A., Puttonen, J. and Yabe, A. (2017) Long Term Application of a Vehicle-Based Health Monitoring System to Short and Medium Span Bridges and Damage Detection Sensitivity. Engineering, 9, 68-122.

https://doi.org/10.4236/eng.2017.92005

Received: December 2, 2016

Accepted: February 13, 2017

Published: February 16, 2017

Copyright (๑) 2017 by authors and Scientific Research Publishing Inc. This work is licensed under the Creative Commons Attribution International License (CC BY 4.0).

http://creativecommons.org/licenses/by/4.0/ (c) (i) Open Access

\begin{abstract}
Largest portion of the bridge stock in almost any country and bridge owning organisation consists on ordinary bridges that has short or medium spans and are now deteriorating due to aging, etc. Therefore, it is becoming an important social concern to develop and put to practical use simple and efficient health monitoring systems for existing short and medium span $(10-30 \mathrm{~m})$ bridges. In this paper, one practical solution to the problem for condition assessment of short and medium span bridges was discussed. A vehicle-based measurement with a public bus as part of a public transit system (called "Bus monitoring system") has been developed to be capable of detecting damage that may affect the structural safety of a bridge from long term vibration measurement data collected while the vehicle (bus) crossed the target bridges. This paper systematically describes how the system has been developed. The bus monitoring system aims to detect the transition from the damage acceleration period, in which the structural safety of an aged bridge declines sharply, to the deterioration period by continually monitoring the bridge of interest. To evaluate the practicality of the newly developed bus monitoring system, it has been field-tested over a period of about four years by using an in-service fixed-route bus operating on a bus route in the city of Ube, Yamaguchi Prefecture, Japan. The verification results thus obtained are also described in this paper. This study also evaluates the sensitivity of "characteristic deflection", which is a bridge (health) condition indicator used by the bus monitoring system, in damage detection. Sensitivity of "characteristic deflection" is verified by introducing artificial damage into a bridge that has ended its service life and is awaiting removal. As the results, it will be able to make a rational long-term health monitoring system for existing short and mediumspan bridges, and then the system helps bridge administrators to establish the rational maintenance strategies.
\end{abstract}




\section{Keywords}

Short and Medium Span Bridge, Long Term Monitoring, Public Bus, Health Monitoring System, Condition Assessment, Damage Detection, Characteristic Deflection, Sensitivity

\section{Introduction}

As an example, many of the bridges in Japan were constructed during the years of rapid economic growth. It is said that the number of bridges ( $2 \mathrm{~m}$ or longer) exceeding 50 years of age will increase in the coming years so that bridges 50 years or older will exceed $65 \%$ of all bridges in the country in 20 years [1] [2] [3] [4]. Furthermore, many of those bridges are short and medium span (about $10 \mathrm{~m}$ to $30 \mathrm{~m}$ or shorter) bridges managed by local governments, and they include many bridges that have deteriorated to the extent of requiring a road closure or traffic restriction. It is therefore necessary to evaluate the structural soundness of so many medium and short span bridges through daily, periodic and other inspections for early detection of bridge anomalies and appropriate maintenance activities. An effective way to achieve this goal is to develop and put to practical use a bridge management system (BMS) mainly for making engineering judgments based on periodic close-range visual inspection data or a structural health monitoring (SHM) system aiming to detect anomalies objectively by use of continuous monitoring data obtained from various sensors. These approaches, however, are being made increasingly difficult by emerging problems such as the shortage of technical experts and cost increase. It is hoped, therefore, that a simple-to-use, efficient bridge monitoring system is developed for short and medium span bridges that will shortly enter the deterioration period in the coming years.

Under these circumstances, growing attention is being paid to the development of the method of detecting bridge damage by evaluating the structural response of the bridge measured when a vehicle equipped with a sensor passes over it [5] [6] [7]. The authors have proposed a bridge monitoring system for short and medium span bridges (called "Bus monitoring system") [8] [9] [10]. Figure 1 illustrates the basic concept and flow of the monitoring method in bus monitoring system. By using an acceleration sensor installed under the rear wheel spring of a bus operating on fixed routes to measure bridge vibration, the bus monitoring system detects the structural damage of the bridge on which the bus is running. For the purposes of damage detection, "characteristic deflection" has been defined as an indicator that may enable efficient detection of structural anomalies of the bridge being monitored [9] [10]. The bus monitoring system (hereinafter referred to as the "System") represents a monitoring method applicable mainly to short and medium span bridges that utilize a fixed-route bus operated as part of a public transport system. The system aims to detect the transition from the damage "acceleration" period, in which the structural safety 


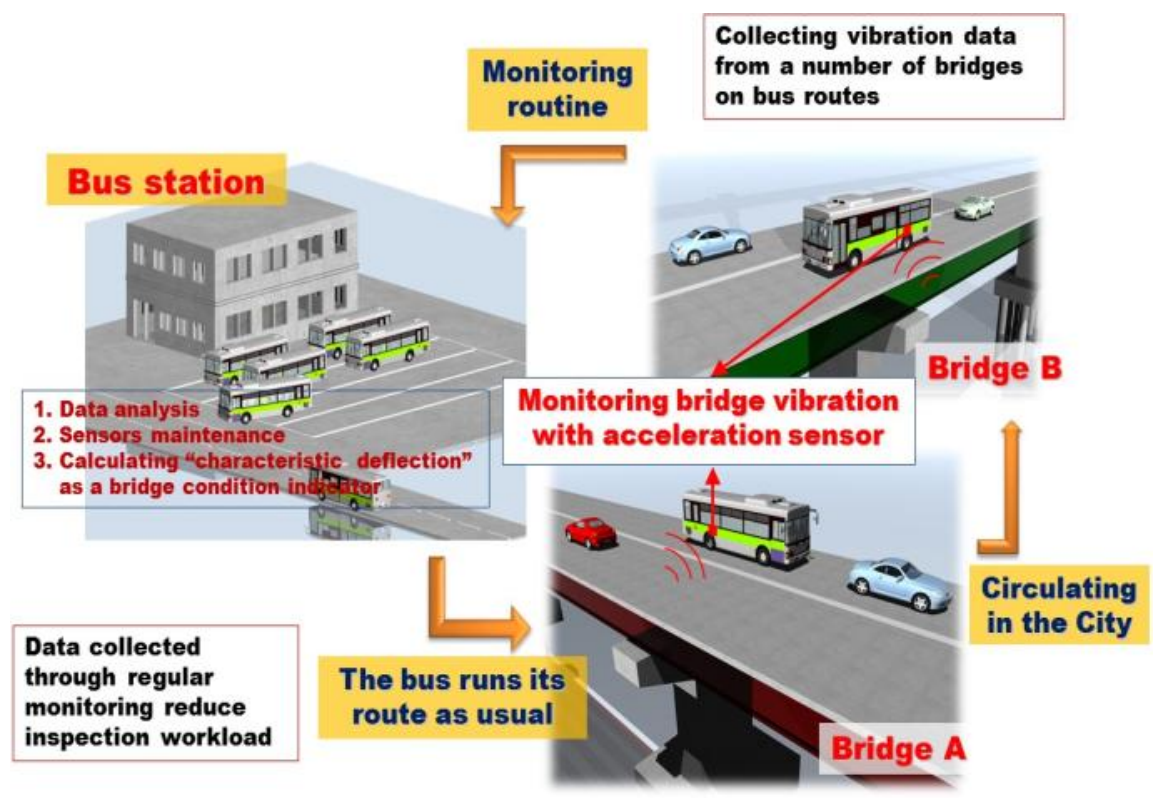

Figure 1. Concept of the bus monitoring system.

of an aged bridge declines sharply, to the "deterioration" period by continually monitoring the bridge of interest (see Figure 2) [9]. Related studies have already done theoretical validation and systematization for the bus monitoring-based damage detection method taking moving loads into consideration; system validation by use of a local fixed-route bus network; and simulations by use of a vibration analysis model taking into consideration factors such as the coupling of a moving vehicle and a bridge [11] [12]. There are still areas, however, where only short-term results have been obtained, such as the evaluation of the influence of conditions peculiar to fixed-route buses (e.g. the number of passengers, vehicle speed, the number of vehicles on the oncoming traffic lane, outdoor air temperature) on detection accuracy and verification by use of real fixed-route bus networks.

In order to solve the remaining problems mentioned above, a long-term field test of the system has been conducted over a period of about four years by using an in-service bus operating in the city of Ube, Yamaguchi Prefecture, Japan and real bridges located on the bus route. This paper described the validation results obtained from the long-term monitoring and discusses the usefulness of the system. Problems of the conventional observation method based on "characteristic deflection", which is a bridge condition indicator that makes possible efficient detection of structural anomalies of the bridge being monitored, are identified, and a new observation method that enhances the damage detection sensitivity of the system is evaluated. This study also examines the influence of artificial damage (guardrail removal) on "characteristic deflection" to evaluate the sensitivity of the system in detecting damage given to the field test bridge. Finally, various study results as mentioned above are put together to systematically discuss the practical scope of application, damage detection accuracy and remaining problems of the system. 


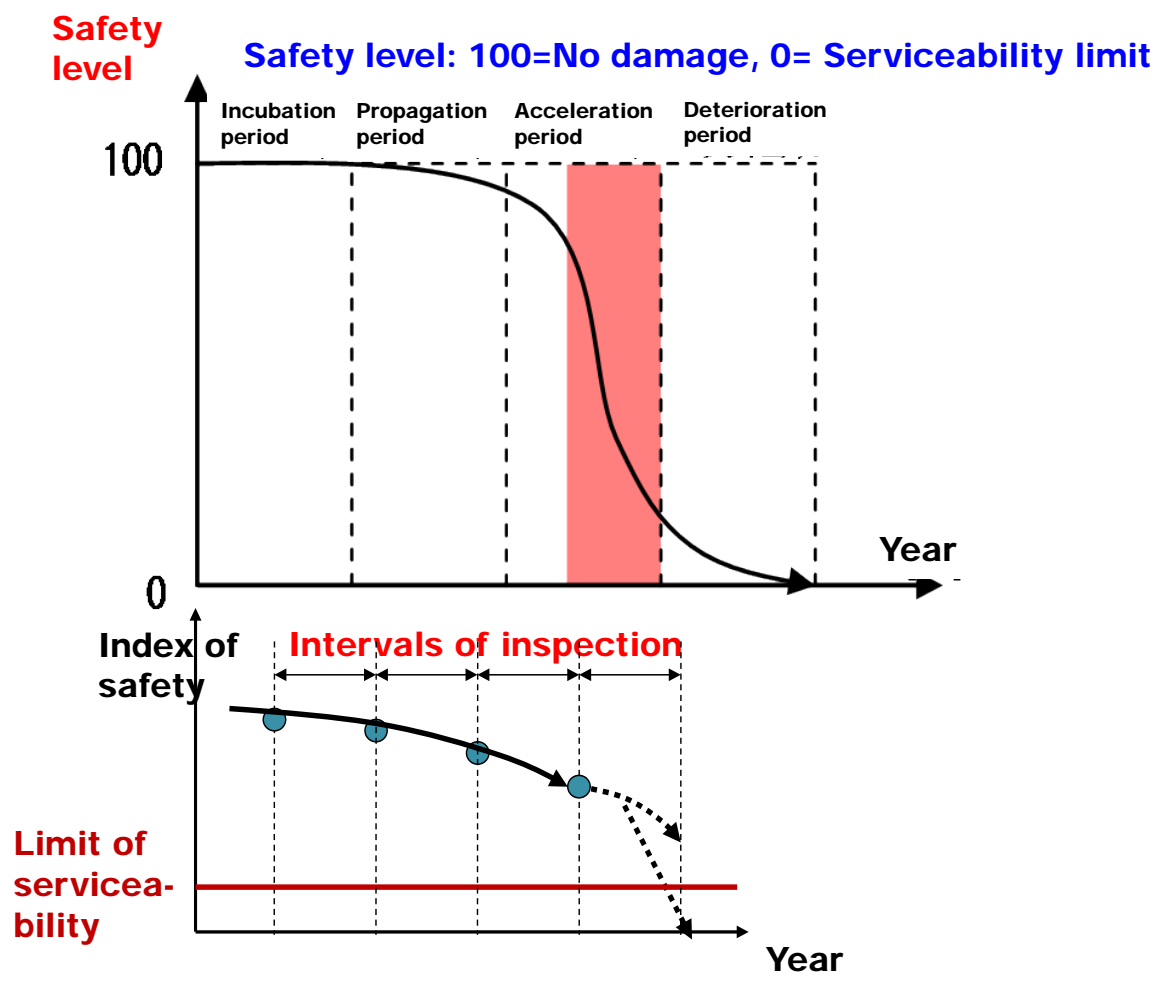

Figure 2. Reduction path of safety level and coverage of proposed system.

\section{Development of a Vehicle-Based Long Term Health Monitoring System-Theoretical Background}

In this chapter, the details of theoretical background and the system are described in detail the flow of the bridge monitoring process (i.e. bus monitoring system) that uses an in-service fixed-route bus as mentioned in the previous chapter. Advantages and principle of the bridge structure anomaly detection (condition assessment) method using the under-spring vibration of an in-service bus (city bus), which has been largely established as a result of the authors' studies, are described in detail. This chapter also describes the procedure for calculating "characteristic deflection," which is an indicator used to evaluate the degree of deterioration (bridge condition) of short and medium span bridges.

\subsection{Overview and Advantages of the Bus Monitoring System}

The aim of the bus monitoring system is to detect anomalies (deterioration) of the bridge of interest by using vibration data, mainly vertical acceleration data, obtained from the acceleration sensor installed under the rear wheel spring of an in-service fixed-route bus. Figure 3 shows the operational flow (simplified) of the bus monitoring system.

Main reasons for having decided to use an in-service fixed-route bus (i.e. a heavy vehicle) are as follows:

a) If a large vehicle about $10 \mathrm{~m}$ long (span) is used for measurement, it is highly likely that when the vehicle crosses a short and medium span bridge that is the only vehicle in the same lane on the bridge. 


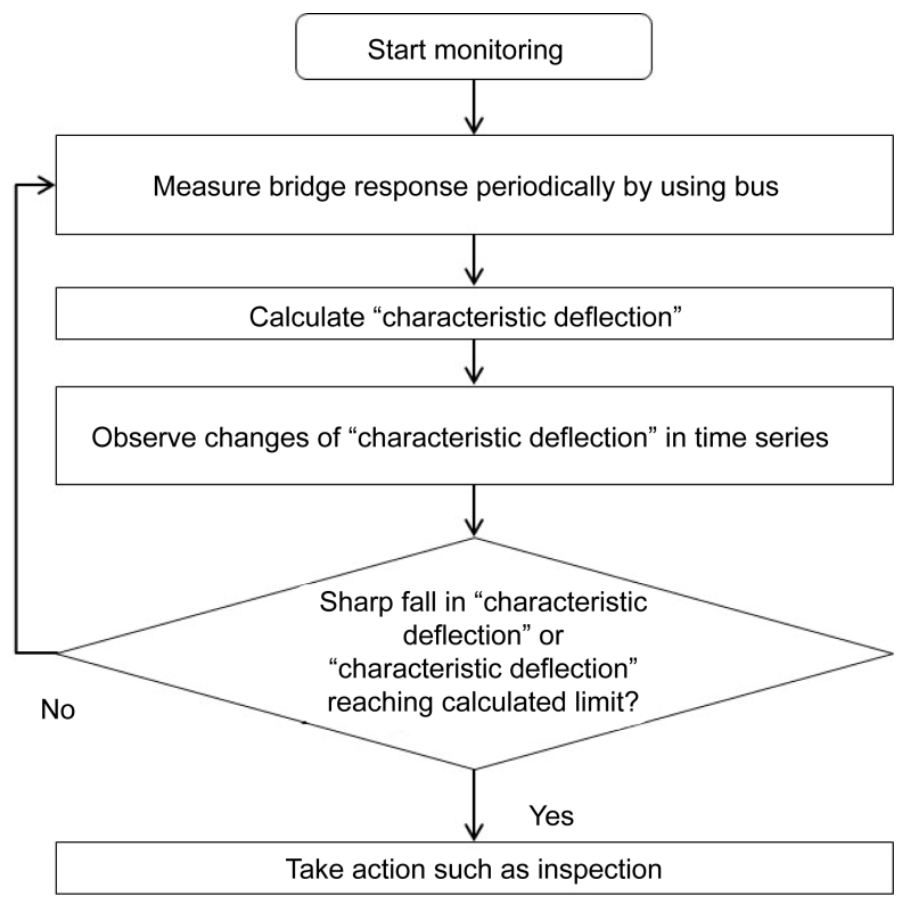

Figure 3. Operational flow of the bus monitoring system.

b) If a short and medium span bridge, which is has relatively high flexural stiffness, is to be vibrated, it is necessary to use a relatively heavy vehicle.

c) If a fixed-route bus is used as a source of bridge excitation, it is easy to reproduce measuring conditions such as the time of passage, route, frequency and velocity.

d) Since a fixed-route bus equipped with a sensor makes the rounds, it is possible to monitor main short and medium span bridges in a particular area on a regular basis. As a result, substantial cost reduction can be achieved because there is no need to install sensors to all bridges to be monitored.

e) The electric power for the measuring instruments used can be supplied by the power supply of the bus.

With regard to the first item, vehicles moving in the opposite direction, or oncoming vehicles, are regarded as an external disturbance factor included in operational conditions in this study (described later). The bus monitoring system is a rational system capable, by using local fixed-route buses, of monitoring bridges on a daily basis while serving as part of transport infrastructure. The bus monitoring system, however, does not identify local deteriorations and their causes because the purpose of the system is to detect damage (anomalies) indicating an overall structural problem of a bridge.

\subsection{Principle of Damage Detection for Bridge Structure Based on the Under-Spring Vibration of a Fixed-Route Bus}

This section describes in detail the principle of operation of the bus monitoring system: how bridge anomalies are detected from vehicle vibration as proposed in a preceding study [13]. 


\subsubsection{Similarity between Bridge Response and the Under-Spring Response of the Bus}

The case in which a vehicle crosses a bridge can be represented by a dynamic interaction between the equation of motion expressed by Equation (1) and the equation of motion expressed by Equation (2). Thus, structural models of the bridge and the vehicle are formulated with different equations of motion, and interactions at points of connection between them are expressed by input and output vectors. This approach is called the "substructure method" [11].

$$
\begin{aligned}
& M_{m} \ddot{\delta}_{m}+C_{m} \dot{\delta}_{m}+K_{m} \delta_{m}=\left\{\begin{array}{c}
F_{s} \\
F_{m}
\end{array}\right\} \\
& M_{s} \ddot{\delta}_{s}+C_{s} \dot{\delta}_{s}+K_{s}\left\{\begin{array}{c}
\delta_{s_{f}} \\
\delta_{s g}
\end{array}\right\}=\left\{\begin{array}{c}
0 \\
F_{s}
\end{array}\right\}
\end{aligned}
$$

where,

$M_{m}, C_{m}, K_{m}$ : mass/damping/stiffness matrix on the bridge side,

$\ddot{\delta}_{m}, \dot{\delta}_{m}, \delta_{m}$ : response acceleration/velocity/displacement matrix on the bridge side,

$M_{s}, C_{s}, K_{s}:$ mass/damping/stiffness matrix on the vehicle side,

$\ddot{\delta}_{s}, \dot{\delta}_{s}, \delta_{s_{f}}:$ response acceleration/velocity/displacement vector on the vehicle side,

$\delta_{s g}$ : input forced displacement vector on the vehicle side,

$F_{m}, F_{s}$ : support reaction vector on the vehicle side.

To express the interaction between the bridge and the vehicle, the underspring reaction of the vehicle is input to the bridge side as load vector, $F_{s}$, and bridge deflection $\left(F_{s} \delta(t) \in \delta_{m}\right)$ and road surface roughness, $\lambda(t)$ are input as forced displacement vector, $\delta_{s g}$. The bridge-vehicle system at time, $t$ to $t+\Delta t$ when the vehicle passes the bridge can be simply represented by a three-massinteraction spring-mass model as shown in Figure 4. The vibration of this system is caused by the vehicle vibration induced by the input of the road surface roughness $(\lambda(t))$ and the bridge deflection, $\delta(t)$, and the excitation to the bridge due to the reaction.

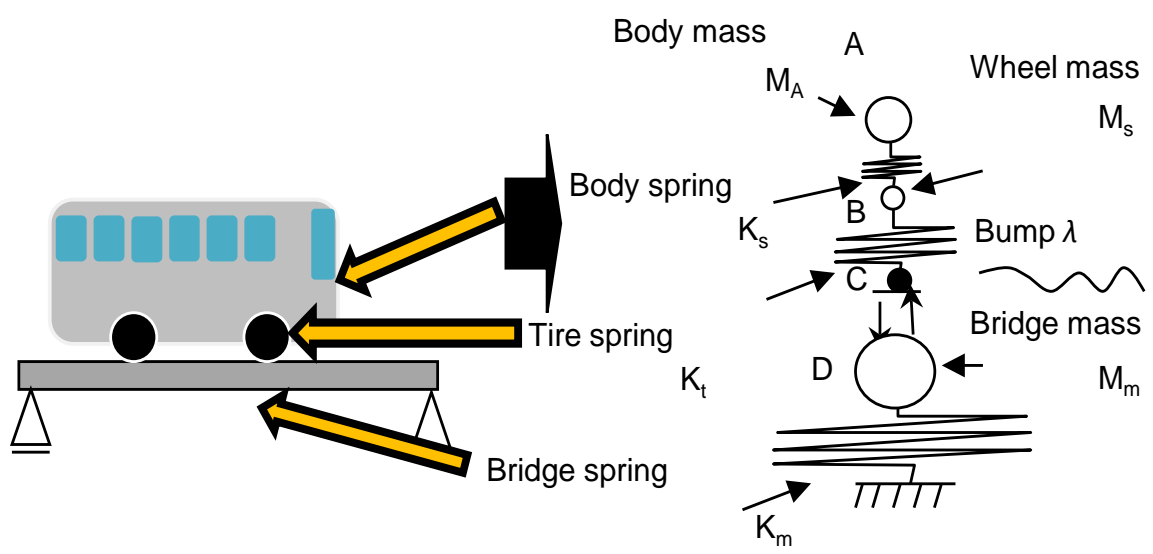

Figure 4. Simple spring-mass model of the bridge-vehicle interaction system. 
The first step is to consider the case where various physical parameters of the bridge system and the vehicle system and the road surface roughness, $\lambda$ remain constant during a certain measurement period. Naturally, the same measurement results are obtained every time from this interaction system.

The next step is to consider the case where various physical parameters for the vehicle system and the road surface roughness, $\lambda$ remain constant and the stiffness, $K_{m}$ of the bridge has changed because of some kind of damage. In this case, the measured value of bridge deflection, $\delta(t)$ due to the reaction from the vehicle system at a given time $t$ also changes. As $\delta(t)$ changes, vehicle system nodal response, $\ddot{\delta}_{s}, \dot{\delta}_{s}, \delta_{s_{f}}$ also changes. Furthermore, as the vibration of the vehicle system changes, vehicle system reaction, that is, exciting force, $F_{s}$ changes so that the bridge deflection, $\delta(t+\Delta t)$ changes. As a result of this chain of changes, effects of the change in the stiffness, $K_{m}$ of the bridge appear in the measurement results obtained from both the bridge system and the vehicle system.

Thus, structural anomalies of the bridge due to deterioration, etc. emerge as changes in vehicle system nodal response, $\ddot{\delta}_{s}, \dot{\delta}_{s}, \delta_{s_{f}}$. It is therefore possible, in theory, to detect bridge anomalies from the vehicle side.

In the case of the proposed system, detection becomes easier as $\delta(t)$ increases. This is why large (heavy) vehicles are more suitable for monitoring than smaller vehicles. According to measurement data [14] [15] in the case of a large vehicle, $M_{A}$ tends to be greater than $M_{S}\left(M_{A}>M_{S}\right)$, and $K_{s}$ tends to be smaller(light) than $K_{t}\left(K_{s}<K_{t}\right)$. This means that the under-spring part (Node B) of the vehicle is more sensitive to changes in the condition of the bridge than the over-spring part (Node A) as shown in Figure 5. For the purposes of this study, therefore, it was decided to pay attention to under-spring vibration. It was also decided to measure acceleration in order to realize a relatively simple vibration measurement system. If bridge vibration is to be estimated from the underspring vibration of the bus, it is necessary to determine how they are correlated.

Let us consider the upper body/lower body/bridge substructuring scheme as shown in Figure 6. The equation of motion for a given system is given in the form of a second-order differential equation:

$$
M \ddot{\delta}(t)+C \dot{\delta}(t)+K \delta(t)=F(t)
$$

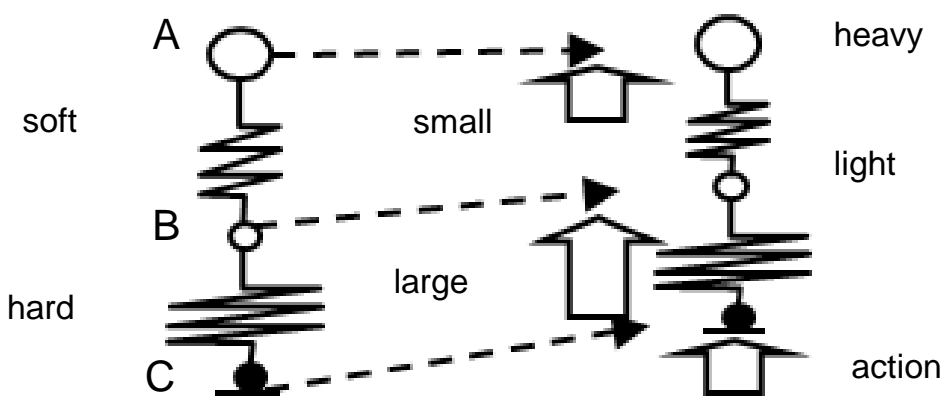

Figure 5. nput to the vehicle system and over-spring and under-spring deformations. 


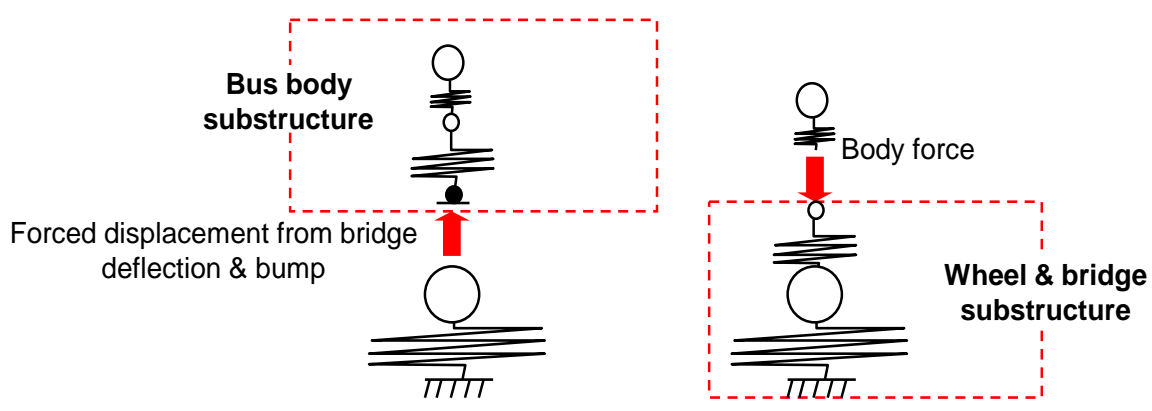

Figure 6. Over-spring/under-spring/bridge substructuring scheme.

where, $M, C$ and $K$ are lumped mass, damping and stiffness matrices for a given system; and $\delta(t), \dot{\delta}(t), \ddot{\delta}(t)$ and $F(t)$ are displacement, velocity, acceleration response and external force vectors, respectively, for a given system at time $t$.

Let differential operator $D$ and shift operator $Z$ be expressed as,

$$
\begin{aligned}
& D \cdot \delta(t)=\frac{\mathrm{d} \delta(t)}{\mathrm{d} t} \\
& Z \cdot \delta(t)=\delta(t+\Delta t)
\end{aligned}
$$

Then, the equation of motion in Equation (3) can be rewritten as,

$$
M \cdot D^{2} \delta(t)+C \cdot D \delta(t)+K \delta(t)=F(t)
$$

Taylor expansion of Equation (5) gives,

$$
\begin{aligned}
\delta(t+\Delta t)= & \delta(t)+\Delta t \frac{\mathrm{d} \delta(t)}{\mathrm{d} t}+\frac{\Delta t^{2}}{2 !} \frac{\mathrm{d}^{2} \delta(t)}{\mathrm{d} t^{2}} \\
& +\frac{\Delta t^{3}}{3 !} \frac{\mathrm{d}^{3} \delta(t)}{\mathrm{d} t^{3}}+\cdots+\frac{\Delta t^{i}}{i !} \frac{\mathrm{d}^{i} \delta(t)}{\mathrm{d} t^{i}}+\cdots
\end{aligned}
$$

Using Equation (4), we can obtain

$$
\delta(t+\Delta t)=\left\{1+\Delta t D+\frac{\Delta t^{2} D^{2}}{2 !}+\frac{\Delta t^{3} D^{3}}{3 !}+\cdots+\frac{\Delta t^{i} D^{i}}{i !}+\cdots\right\} \delta(t)
$$

If Newmark's $\beta$ method $(\beta=1 / 4)$ is used, the differential operator relation can be assumed as follows:

$$
D \Delta t \fallingdotseq \frac{2\left(1-Z^{-1}\right)}{1+Z^{-1}}
$$

Substituting this in Equation (6) gives Equation (10):

$$
M \cdot\left(\frac{\Delta t}{2} \cdot \frac{\left(1-Z^{-1}\right)}{1+Z^{-1}}\right)^{2} \delta(t)+C \cdot\left(\frac{\Delta t}{2} \cdot \frac{\left(1-Z^{-1}\right)}{1+Z^{-1}}\right) \delta(t)+K \delta(t)=F(t)
$$

Let $k$ represent a post-discretization step at time $t$, and $k+1$, the next step. Then, Equation (5) can be rewritten as $Z \cdot x(k)=x(k+1)$. Hence, Equation (10) can be reduced to the difference equation: 


$$
\begin{aligned}
& \left\{M+\frac{\Delta t}{2} C+\left(\frac{\Delta t}{2}\right)^{2} K\right\} \delta(k)-\left\{M-\left(\frac{\Delta t}{2}\right)^{2} K\right\} \delta(k-1) \\
& +\left\{M-\frac{\Delta t}{2} C+\left(\frac{\Delta t}{2}\right)^{2} K\right\} \delta(k-2) \\
& =\left(\frac{\Delta t}{2}\right)^{2}\{F(k)+2 F(k-1)+F(k-2)\}
\end{aligned}
$$

The right-hand side and the second and third terms of the left-hand side of Equation (11) are known when solving the equation at step $k$ (=time $t$ ). Let $F^{\prime}(t)$ represent the right-hand side of the equation; $C_{0}(M, C, K, t)$, the second and third terms of the left-hand side; and $P(M, C, K, t)$, the coefficient of $\delta(k)$ of the first term of the left-hand side. Then, Equation (11) can be rewritten as,

$$
\delta(t) \cdot P(M, C, K, t)+C_{0}(M, C, K, t)=F^{\prime}(t)
$$

where, $P(M, C, K, t)$ is a proportionality coefficient dependent on the system at time $t$. On the assumption that difference approximation is valid, the equation of motion for the wheel-bridge system can be written, by using the proportionality coefficient $P$ and the state constant, $C_{0}$ (known value), as shown in Equation (12) [16]. This means that the response to the input vector is distributed proportionately depending on system-dependent constants.

Next, let us consider the vibration of the wheel-bridge system due to the force transmitted from the upper part of the vehicle.

As in the case mentioned earlier, the response of the wheel-bridge system to the input from the upper part of the vehicle is distributed proportionately depending on physical constants of the system. It can be inferred, therefore, that if $A_{b}$ represents the bridge response vector and $A_{s}$ represents the bus wheel response vector, then matrix $P$ that satisfies the following equation under continuously changing conditions:

$$
A_{b}=P^{-1} A_{s} P
$$

\subsubsection{Extracting Damage and Deterioration Related Information from Under-Spring Vibration of the Bus}

This section describes the concept of the method of extracting damage and deterioration related information from the vertical under-spring vibration of the bus without being affected by the dynamic characteristics of the bridge and the vehicle and road surface roughness. The vertical under-spring vibration response, $\delta_{a}(t)$ of a bus traveling at a constant speed can be expressed as the sum of static displacement, $\delta_{s a}(t)$, which is dependent on the stiffness of the bridge and the weight of the bus, and dynamic displacement, $\delta_{d a}(t)$, which is dependent on road surface roughness and the vibration characteristics of the bridge and the vehicle:

$$
\delta_{a}(t)=\delta_{s a}(t)+\delta_{d a}(t)
$$

If road surface roughness is assumed to be a stationary random Gaussian 
process with a mean value of 0 and if dynamic displacement including the bridge-vehicle interaction is assumed to be an ergodic process and therefore Fourier-expandable, the dynamic displacement, $\delta_{d a}(t)$ can be expressed as their sum:

$$
\delta_{d a}(t)=S_{r}(\Omega, t)+\int_{-\infty}^{+\infty} X(f) \cdot \mathrm{e}^{2 \pi f t i} \mathrm{~d} f
$$

where, $S_{r}(\Omega, t)$ is a density function for road surface roughness; $\Omega$, the spatial frequency of the road surface; and $X(f)$, a Fourier series. In Equation (15), the limit of the sample average of the second term is 0 . The mean value, therefore, of $N$ samples, where $N$ is a sufficiently large number, obtained from $\delta_{d a}(t)$ can be expressed as,

$$
\frac{\sum_{t=1}^{N} \delta_{d a}(t)}{N} \fallingdotseq 0
$$

As the next step, a total of $k$ samples are taken from measured values of $\delta_{a}(t)$, and their mean value can be expressed, by representing their mean by $\overline{\delta_{a}(t)}$, as,

$$
\overline{\delta_{a}(t)}=\frac{\sum_{j=1}^{k} \delta_{a}(j)}{k}
$$

Since the distribution of sample means should be normal according to the central limit theorem, $\overline{\delta_{a}(t)}$ should converge to a certain value, $\mu_{a}$. For a sufficient number $(n)$ of sample means $\delta_{a}(j)$, therefore, it can be expressed as,

$$
\mu_{a}=\frac{\sum_{i=1}^{n} \overline{\delta_{a}(t)_{i}}}{n}=\frac{\sum_{i=1}^{n k}\left(\delta_{a}(j)\right)_{i}}{n k}
$$

If sampling from $\delta_{a}(t)$ is done so as to avoid duplication and $N$ is sufficiently large relative to $n k=N$ and $t=1$ to $N$, then the following approximation can be made:

$$
\mu_{a} \fallingdotseq \frac{\sum_{t=1}^{N} \delta_{a}(j)_{t}}{N}
$$

This expresses the average vertical under-spring displacement of a bus crossing a bridge. This can be rewritten, on the basis of Equations (14) and (16), as,

$$
\mu_{a} \fallingdotseq \frac{\sum_{t=1}^{N} \delta_{s a}(t)}{N}
$$

This means that the average of sample values obtainable from a sufficiently large number $(N)$ of measured values of vertical under-spring displacement of a bus crossing a bridge can be extracted as values $\left(\mu_{a}\right)$ that are relatively unaffected by the vibration characteristics of the bridge and the vehicle and the dynamic displacement due to road surface roughness. The $\mu_{a}$ thus obtained is referred to as "characteristic deflection".

Means of deflection, $\delta_{b}(t)$ at a given point on the bridge when a vehicle crosses it also converge to a certain value, $\mu_{b}$, relatively unaffected by dynamic deflection by making similar assumptions. In a similar way, $\mu_{b}$ can be expressed, 
by using static deflection, $\delta_{s b}(t)$, as,

$$
\mu_{b} \fallingdotseq \frac{\sum_{t=1}^{N} \delta_{s b}(t)}{N}
$$

If the law of similarity mentioned earlier holds true with respect to $\delta_{s a}(t)$ and $\delta_{s b}(t)$, they can be related as,

$$
\mu_{b}=P \mu_{a}
$$

Let $\mu_{a}$ and $\mu_{b}$ represent values extracted from values obtained from measurement when the bridge is in a sound condition and $\mu_{a}^{\prime}$ and $\mu_{b}^{\prime}$ represent values extracted from values obtained after the occurrence of deterioration or damage. Then, the change ratio, $\alpha$ expressed as,

$$
\alpha=\frac{\mu_{b}^{\prime}}{\mu_{b}}=\frac{\mu_{a}^{\prime}}{\mu_{a}}
$$

And it can be defined as a parameter for structural anomaly detection. After setting the value of $\alpha$, "characteristic deflection" is monitored, and if it has exceeded a certain limit, it can be deemed to indicate that the latter half of the damage acceleration period of the bridge has ended and the bridge has entered the deterioration period. In reality, however, bus operation is affected by not only the static displacement, $\delta_{\text {sa }}(t)$ and the dynamic displacement, $\delta_{\text {da }}(t)$ expressed by Equation (14) but also external disturbance factors, $\delta_{x}(t)$, such as weather and oncoming vehicles. For the purposes of this study, the vertical displacement, $\delta_{a}(t)$ including the influence of external disturbance factors, $\delta_{x}(t)$ is expressed as

$$
\delta_{a}(t)=\delta_{s a}(t)+\delta_{d a}(t)+\delta_{x}(t)
$$

and its characteristics and actions to be taken are considered by using a real in-service fixed-route bus.

\subsubsection{Characteristic Deflection Calculation Method}

Figure 7 shows the flow of "characteristic deflection" calculation after the acquisition of measurement data from the acceleration sensor installed to the bus. Each step is described below in detail. The step numbers ("Step 1" to "Step 5") shown below correspond to the numbers shown in Figure 7.

\section{Step 1: Extract data on vertical acceleration during bridge crossing}

Data on vertical acceleration during bridge crossing are extracted from acceleration sensor measurement data by referring to a combination of other data such as the time at which the buses crossed each bridge and GPS data.

\section{Step 2: Estimate the time at which the midspan point was passed}

Extracted data on acceleration during bridge crossing include considerable vibrations recorded at joints. It is therefore necessary to use midspan acceleration data that do not include such joint vibrations. The time at which the midspan point of a girder was passed can be estimated by identifying bridge sections meeting such criteria as duration and wave count and extracting relevant data from non-joint data. It may be difficult, however, to identify joint locations 


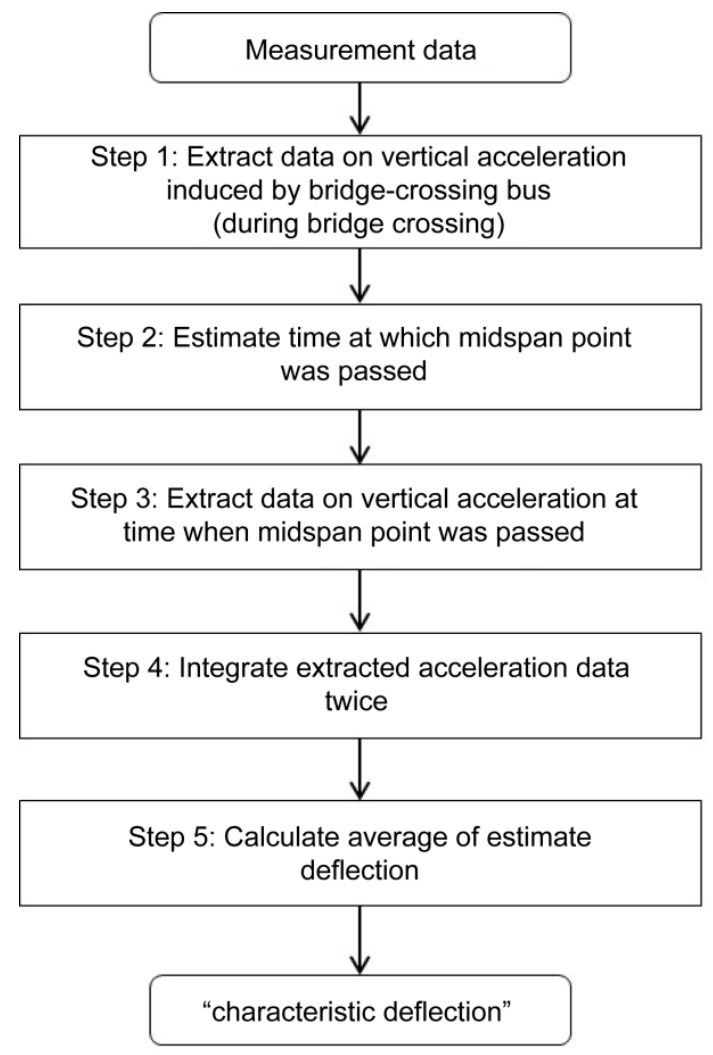

Figure 7. "Characteristic deflection" calculation flow.

because acceleration data may vary in magnitude depending on such factors as bus operating conditions. For accurate estimation of the time at which the midspan point was passed, therefore, attention is paid to estimated deflection diagrams obtained by integrating vertical acceleration data twice. As an example, Figure 8 shows acceleration waveforms measured recently and estimated deflection waveforms obtained by integrating the bridge-crossing acceleration data twice. As shown in Figure 8, characteristic waveforms appear when the bus crosses a bridge. The waveforms at midspan, therefore, are estimated and identified by synchronizing different data on the basis of the characteristic waveforms appearing in the estimated deflection diagram when the bus passes the joints of the bridge of interest.

Step 3: Extract data on vertical acceleration during bridge crossing

Extract the midspan vertical acceleration data identified at Step 2. The most important thing in "characteristic deflection" calculation is to determine the extraction range according to such details as wave count and duration and extract acceleration waveform data from the same segment in every time. Step 3 is described in detail in the next section.

\section{Step 4: Integrate the extracted acceleration data twice}

The extracted acceleration data is converted to velocity data by integrating once and to displacement data by integrating twice. In this study, the vertical displacement obtained by integrating the vertical acceleration twice is regarded as estimated midspan deflection during bridge crossing. Figure 9 shows an example 


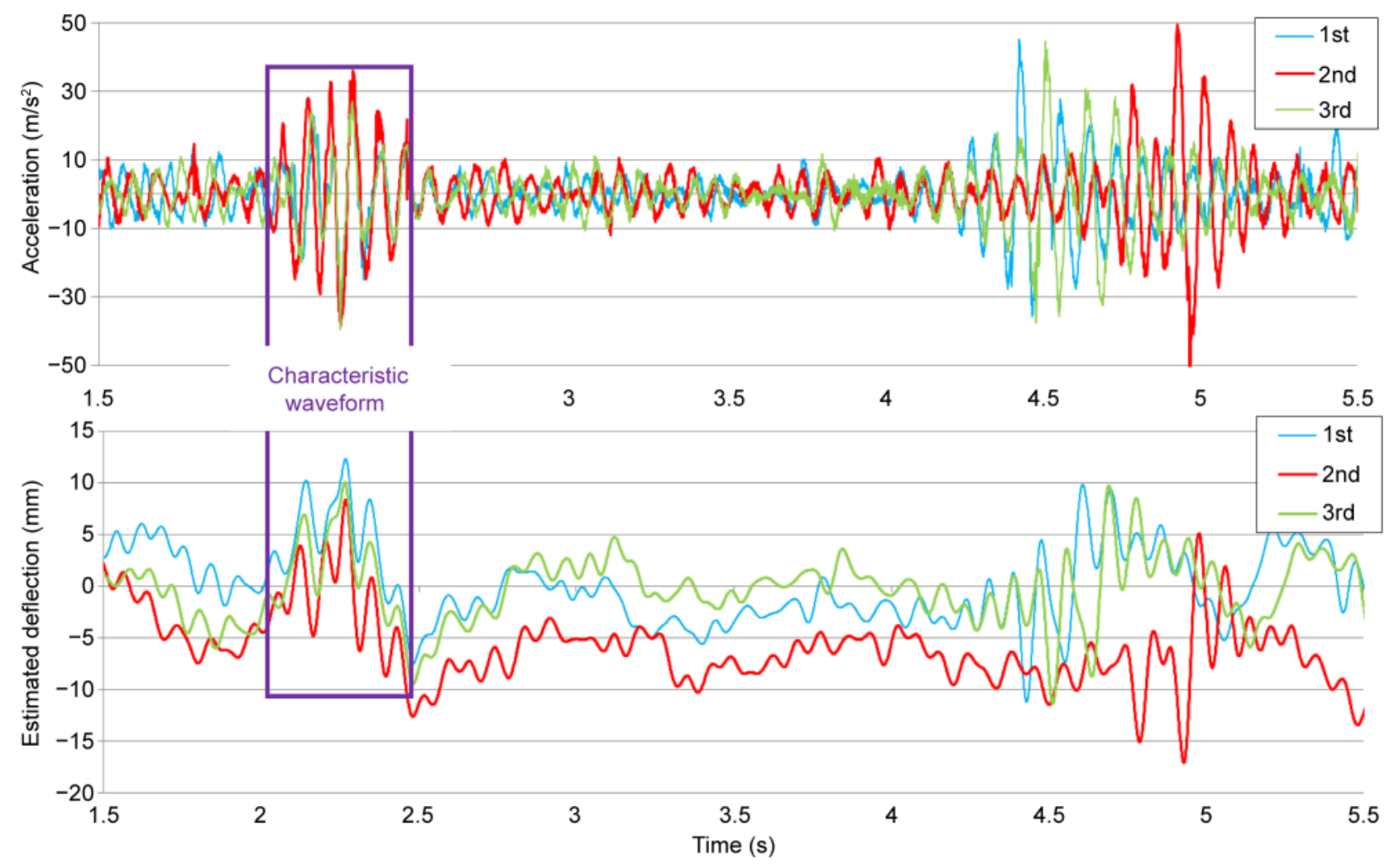

Figure 8. Examples of characteristic waveforms of estimated deflection.

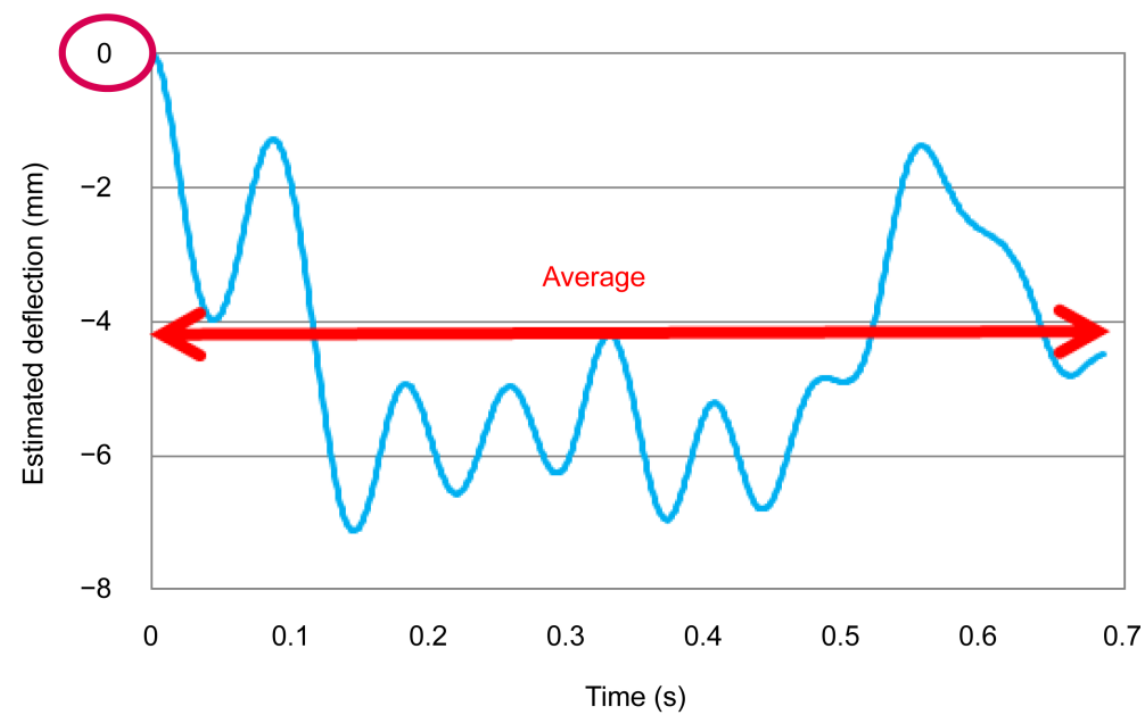

Figure 9. Example of estimation of deflection.

of estimated deflection in this way. As shown in Figure 9, the vertical displacement (estimated deflection) at time 0 (shown with a red circle) was assumed to be the initial value of 0 in the selected midspan section.

Step 5: Average estimated deflections

The estimated midspan deflections during bridge crossing shown in the graph are time-averaged to calculate the "characteristic deflection" (see Figure 9). 
Described above is the procedure for calculating the "characteristic deflection" used as an indicator in the proposed evaluation method. "Characteristic deflections" thus calculated include the effects of external disturbance factors as shown in Equation (24). It has been confirmed that "characteristic deflections" calculated as described above are significantly affected by human errors (individual errors). Efforts need to be made, therefore, to minimize human error in the calculation process.

\section{Application to Long Term Monitoring of Short and Medium Span Bridges on Bus Routes in Ube City, Japan}

This chapter describes on the long-term field test of the bus monitoring system for short and medium span bridges located on the municipal bus routes in the city of Ube, Yamaguchi Prefecture, Japan conducted over a period of about four years from December 2010 to September 2014. Since the field test has been conducted for about four years, a considerable amount of measurement data has been accumulated. The data thus accumulated were utilized to evaluate the influence of fixed-bus operating conditions (external disturbance factors such as weather, the number of oncoming vehicles, the number of persons in the vehicle and vehicle speed) on characteristic deflection. In addition to the derivation of conversion (correction) factors based on the correlations between various bus operating conditions (external disturbance factors) and characteristic deflection carried out in previous studies [9] [10] based on data accumulated over a period of about one year, newly obtained measurement data were used. Thus, by conducting an integrated study using all data accumulated over a combined period of about four years, new study results have been obtained. These results are also reported in this chapter.

\subsection{Overview of Long-Term Monitoring Conducted on Ube-City's Bus Routes}

In order to develop and put to practical use a bus monitoring system for short and medium span bridges located on bus routes, it is necessary to conduct a series of studies involving a long-term field test using an in-service fixed-route bus. In this study, with the cooperation of Ube-city's Transportation Bureau (UTB), long-term monitoring of short and medium span bridges located on the city's in-service bus routes has been continued. The study focuses mainly on the following:

(1) The number of short and medium span bridges existing on the city's bus routes and the total number of existing bridges in need of maintenance.

(2) The method of calculating "characteristic deflection," which is an indicator of the structural health of bridges based on long-term measurement data and its usefulness in damage detection.

(3) Proposing a method for long-term observation of characteristic deflection and enhancing damage detection sensitivity.

(4) Verifying the damage detection sensitivity of characteristic deflection by 
use of artificial damage and setting "critical characteristic deflection" (criterion value) by use of an analysis model.

(5) Evaluation of the influence of bus operating conditions (external disturbance factors) on characteristic deflection based on long-term measurement data and an attempt at deriving conversion (correction) factors.

\subsubsection{Number of Bridges on Municipal Bus Routes and Bridges to Be Monitored}

Figure 10 shows the number of bridges under the jurisdiction of Ube-city located on the municipal bus routes [17] [18] operated by Ube-city's Transportation Bureau. Of a total of 435 bridges $2 \mathrm{~m}$ or more in length managed by Ubecity, 35 bridges are located on the bus routes. Although they account for less than $10 \%$ of all bridges in the city, after the bus monitoring system goes into service, all existing bridges in need of maintenance will be monitored with the cooperation of Ube-city's Construction Department. Prior to the long-term field test using the bus monitoring system, short and medium span bridges to be monitored in the test were selected. Figure 11 shows the present and future of the bridges managed by Ube-city's Construction Department, comparing the percentages of bridges older than 50 years. As shown, the number of bridges

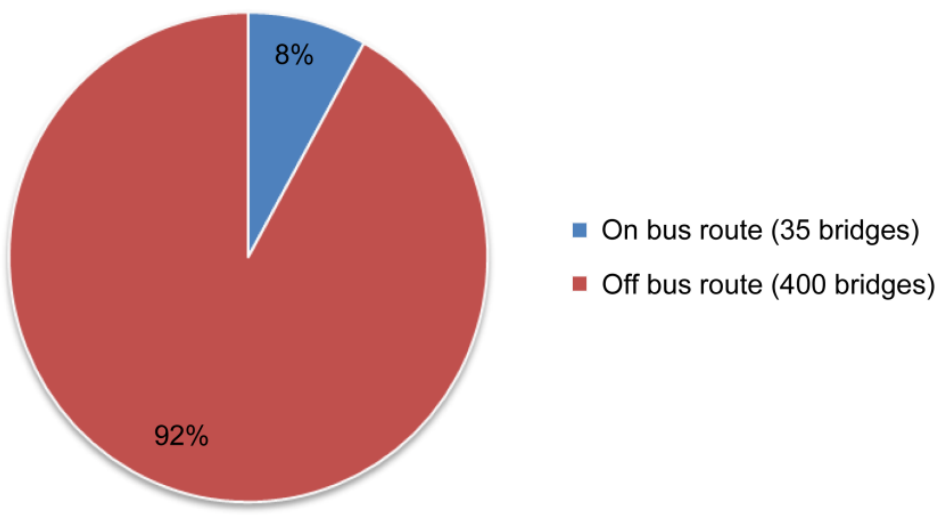

Figure 10. Number of bridges managed by Ube-city Transportation Bureau (UTB) located on bus routes.

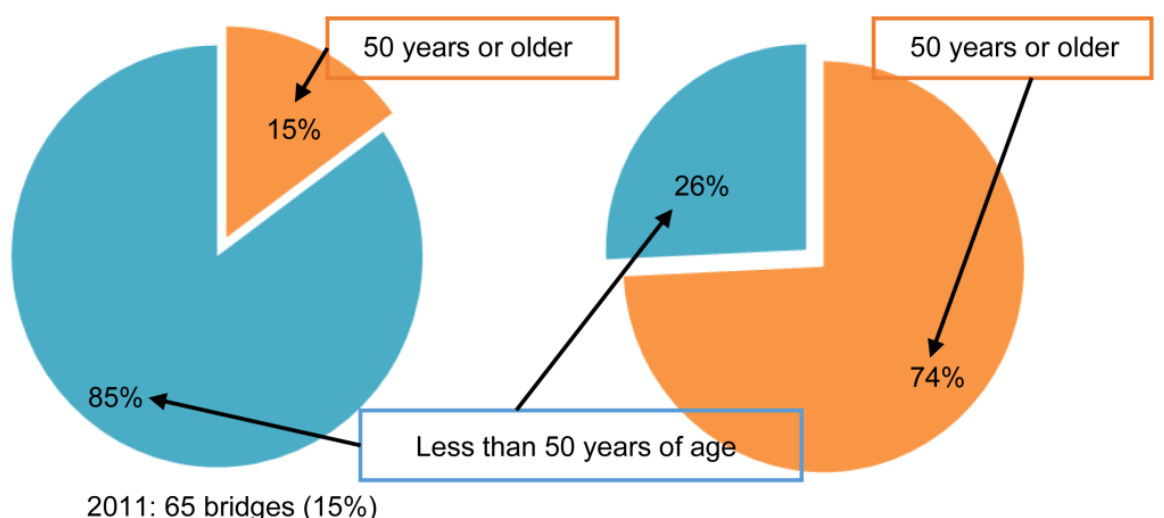

Figure 11. Percentage of bridges 50 years or older in all bridges in Ube-city 20 years from now. 
older than 50 years as of fiscal year 2011 is 65 , which is about $15 \%$ of all bridges. In 2031 (20 years later), it will increase to 323 bridges (about $74 \%$ of all bridges), indicating a rapid deterioration of the bridges in the city. Of such short and medium span bridges located on the bus routes in Ube-city, three bridges that are thought to have deteriorated considerably, namely, "Shiratsuchi Daini Bridge (2-span RC T-girder bridge)", "Jase Bridge (5-span PC slab bridge)" and "Shingondai Bridge (single-span prestressed concrete girder bridge built by the BiPrestressing Method)", were selected for the long-term monitoring. Specifications and general views of these three bridges are shown in Table 1 and Figure 12 , respectively.

\subsubsection{Overview of the Vehicle Used for Long-Term Measurement}

This section briefly describes the bus (vehicle) used for the long-term field test. The long-term measurement using Ube-city's municipal bus routes has been continued by using an in-service fixed-route bus (i.e. a bus actually used to transport passengers) owned by Ube-city's Transportation Bureau. By using the three-axis acceleration sensor installed under the rear wheel spring of this

Table 1. Data on bridges selected for long-term monitoring.

\begin{tabular}{|c|c|c|c|c|c|c|}
\hline Bridge name & Completed in & \multicolumn{3}{|c|}{ Type of superstructure } & Span length $(\mathrm{m})$ & Bridge length $(\mathrm{m})$ \\
\hline \multirow{5}{*}{ Jase Bridge } & \multirow{5}{*}{1976} & \multirow{5}{*}{ Span No. } & Start point side 1 & $\begin{array}{l}\text { Prestressed concrete slab bridge } \\
\text { (pretensioned slab) }\end{array}$ & 18.0 & \multirow{5}{*}{85.0} \\
\hline & & & 2 & $\begin{array}{l}\text { Prestressed concrete slab bridge } \\
\text { (pretensioned slab) }\end{array}$ & 16.0 & \\
\hline & & & 3 & $\begin{array}{l}\text { Prestressed concrete slab bridge } \\
\text { (pretensioned slab) }\end{array}$ & 18.0 & \\
\hline & & & 4 & $\begin{array}{l}\text { Prestressed concrete slab bridge } \\
\text { (pretensioned slab) }\end{array}$ & 14.0 & \\
\hline & & & End point side 5 & $\begin{array}{l}\text { Prestressed concrete slab bridge } \\
\text { (pretensioned slab) }\end{array}$ & 19.0 & \\
\hline \multirow{2}{*}{$\begin{array}{l}\text { Shiratsuchi } \\
\text { Daini Bridge }\end{array}$} & \multirow{2}{*}{$\begin{array}{c}1933 \\
\text { (estimated) }\end{array}$} & \multirow{2}{*}{ Span No. } & Start point side 1 & Reinforced concrete (T-girder) & 7.0 & \multirow{2}{*}{15.0} \\
\hline & & & End point side 2 & Reinforced concrete (T-girder) & 7.0 & \\
\hline $\begin{array}{l}\text { Shingondai } \\
\text { Bridge }\end{array}$ & June 1998 & \multicolumn{3}{|c|}{$\begin{array}{l}\text { Single-span prestressed concrete girder bridge } \\
\text { (Bi-prestressing method) }\end{array}$} & 22.4 & 23.6 \\
\hline
\end{tabular}

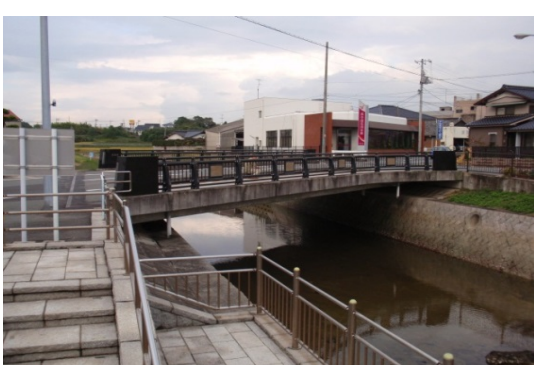

(a)

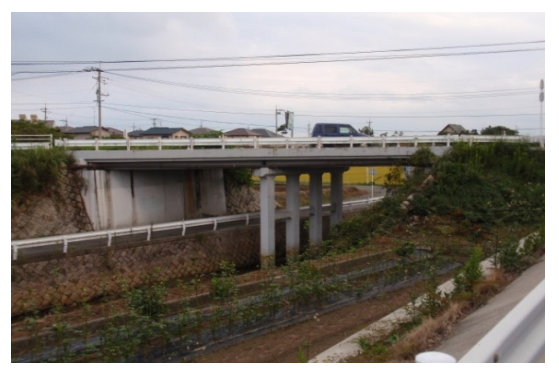

(b)

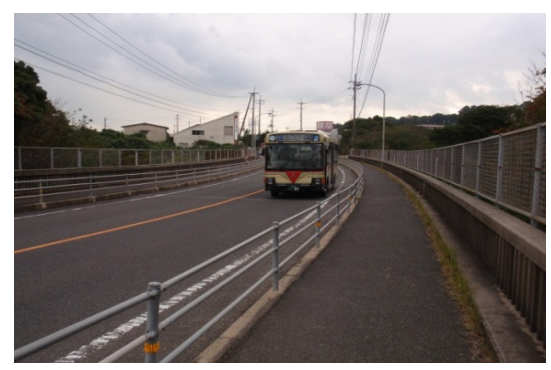

(c)

Figure 12. General views of Ube-city bus route bridges selected for long-term monitoring. (a) Shingondai Bridge (single-span brige); (b) Shirasuchi Daini Bridge (two-span); (c) Jase Bridge (five-span bridge). 
vehicle, the vibration properties of the bridge being crossed by the bus were extracted as the acceleration response during bridge crossing and deflection was estimated [9]. Table 2 and Table 3 show the specifications [19] of the bus (vehicle) and the three-axis acceleration sensor installed to the bus, respectively.

Figure 13 shows a general view of the bus (vehicle) used for the long-term monitoring. Figure 14(a) shows the acceleration sensor installed under the rear wheel spring of the vehicle. The acceleration sensor was bonded to the underside of the rear wheel spring and was coated with waterproof epoxy resin to protect the sensor over a long period of time. The three-axis acceleration sensor used was positioned so that its $\mathrm{X}, \mathrm{Y}$ and $\mathrm{Z}$ axes were aligned with the direction of travel of the bus, the direction perpendicular to the direction of travel and the vertical direction, respectively. Analog vibration data obtained from the threeaxis acceleration sensor in the form of acceleration response were converted to digital data via a data logger and saved in the computer as Excel file data. As shown in Figure 14(b), the cable from the three-axis acceleration sensor was routed through the drain hole in the vehicle floor to the data logger.

\subsubsection{Measuring Method}

Figure 15 illustrates the measuring method adopted for the bus monitoring system. In the long-term monitoring that has been conducted on the bus routes

Table 2. Specifications of bus (vehicle) used for long-term monitoring.

\begin{tabular}{cc}
\hline Item & Specifications \\
\hline Net vehicle weight & $8130 \mathrm{~kg}$ \\
Gross vehicle weight & $11,485 \mathrm{~kg}$ \\
Front axle weight & $2730 \mathrm{~kg}$ \\
Rear axle weight & $5400 \mathrm{~kg}$ \\
Wheel base & $4.4 \mathrm{~m}$ \\
\hline
\end{tabular}

Table 3. Specifications of 3-axis acceleration sensor used for long-term monitoring (vehicle side).

Name: Fuji Ceramics SA11ZSC-TI

(Three-axis piezoelectric acceleration transducer with built-in amplifier)

$\begin{array}{lc}\text { Charge sensitivity } & 1 \mathrm{mV} / \mathrm{m} / \mathrm{s}^{2} \\ \text { Frequency range } & 1-8000 \mathrm{~Hz} \\ \text { Resonant frequency } & 35 \mathrm{kHz} \text { or higher } \\ \text { Maximum measurable acceleration } & 4000 \mathrm{~m} / \mathrm{s}^{2} \\ \text { Maximum allowable acceleration } & 30,000 \mathrm{~m} / \mathrm{s}^{2} \text { or higher } \\ \text { Power supply for built-in amplifier } & 21-24 \mathrm{~V} / 0.5-10 \mathrm{~mA} \\ \text { Temperature range } & -50-+110^{\circ} \mathrm{C} \\ \text { Dimensions } & 14.2 \times 14.2 \times 14.2 \mathrm{~mm} \\ \text { Mass } & \text { Approx. } 11.1 \mathrm{~g}\end{array}$




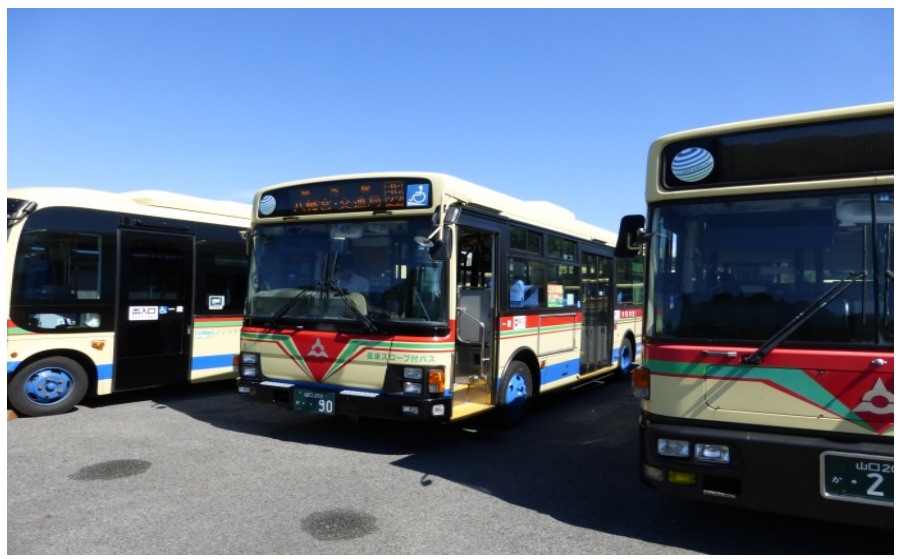

Figure 13. General view of the bus (vehicle) used for the bus monitoring system.

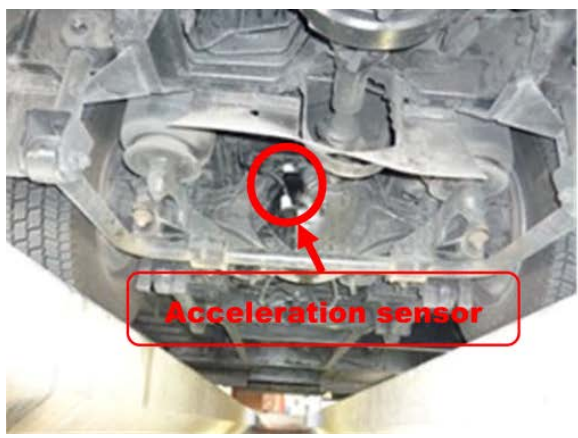

(a)

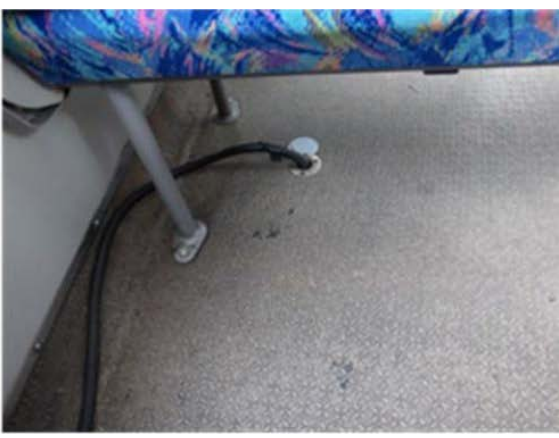

(b)

Figure 14. Installation of acceleration sensor to the bus (vehicle). (a) Acceleration sensor installed to the rear wheel of the bus; (b) Wiring routes into the bus.

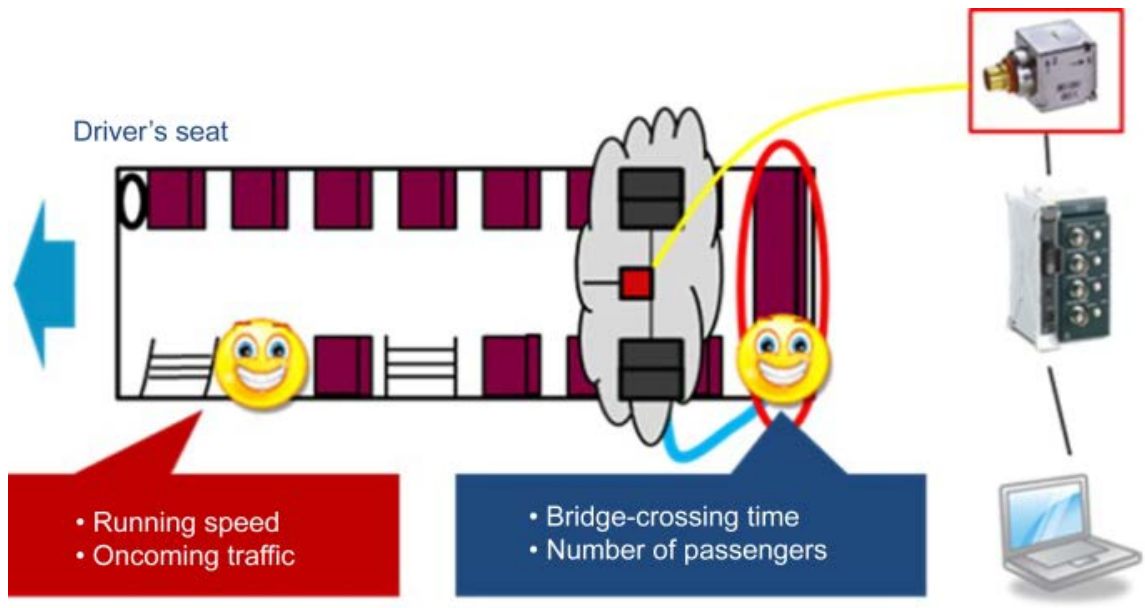

Figure 15. Configuration of the on-board measurement system of the bus monitoring system.

in Ube-city, attempts were made to systematically evaluate the influence of bus operating conditions (weather, the number of oncoming vehicles, the number of persons on the vehicle and vehicle speed), besides the acceleration response recorded with the three-axis acceleration sensor, on characteristic deflection and elucidate and quantify their correlations. During the data measurement, a two- 
person measuring team rode on the bus. One of them, who sat on a front seat near the bus driver, recorded details such as vehicle speed, the number of oncoming vehicles (if any) and weather conditions. The other person, who sat on a rear end seat, operated and checked on the measuring equipment and recorded the number of persons on the bus and the time at which the bus crossed the bridge in time series while collecting other information on possible external disturbance factors. Figure 16 shows the types of equipment and devices used and how they looked. As shown in Figure 16, by connecting the three-axis acceleration sensor with the measuring and display equipment, the person at the rear end seat was enabled to monitor vibration waveforms in real time. The plan for the operation of the bus monitoring system in the coming years assumes the use of the power supply of the bus for the acceleration sensor, data logger and the computer. In the measurement reported in this study, however, a portable battery was used (see Figure 16).

\subsection{Results of On-Site Verification Examined in Previous Studies [9] [10]}

Before reporting the results of the long-term field test conducted over a period of about four years, this section touches on some fundamental findings from previous studies. First, a study was conducted to determine whether it is possible to extract the "estimated deflection" (basic data for the calculation of "characteristic deflection") of the bridge of interest from the rear wheel under-spring acceleration response of a bus (vehicle). In that study, an acceleration sensor was installed in the midspan zone of the Shingondai Bridge (prestressed concrete girder bridge built by the Bi-Prestressing Method), which is one of the three
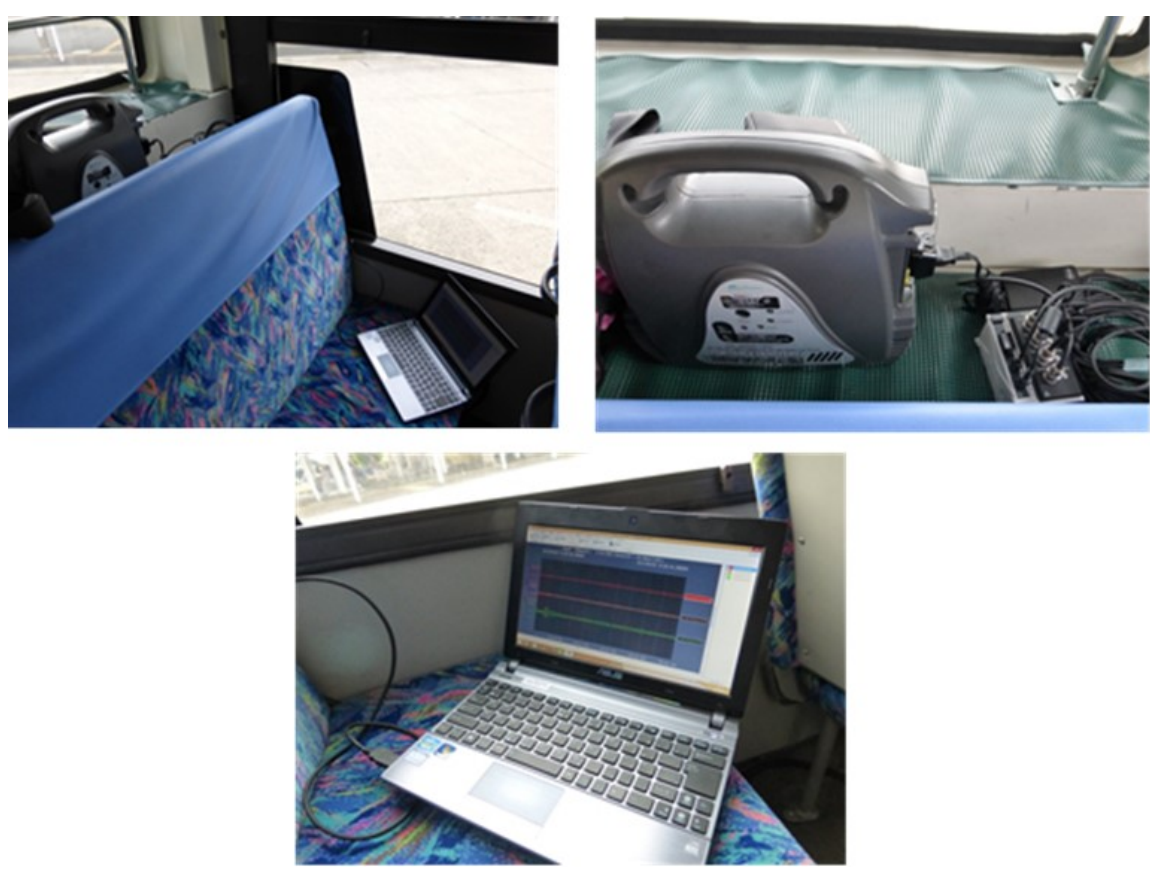

Figure 16. Measurement in progress in the bus and the measuring equipment used (back of the bus). 
bridges selected for the present study, and the acceleration response of the bridge and the under-spring acceleration response of the bus were compared in time series. Next, another study was conducted to evaluate the influence of bus operating conditions during bridge crossing on characteristic deflection and use the findings for conversion (correction) factor derivation in future. In the study, coefficients of correlation between those conditions and characteristic deflection were derived. Although the goal of conversion (correction) by use of correlation coefficients was not achieved because the required amount of data was not available, the study succeeded in showing that the variability of characteristic deflection can be reduced by applying the moving average method to a time series. A vehicle-induced vibration simulation taking account of the coupling with the bus and the bridge, etc. was also performed by using the substructure method [20], which is a technique classified as a finite element method (FEM). The aim of the simulation was to develop "serious deterioration (damage) criteria" by which to determine the degree of increase in "characteristic deflection" that can be deemed to be the onset of serious damage leading to the transition to the deterioration phase of a bridge.

\subsubsection{Time Series Comparison of Rear Wheel Acceleration Response of the Bus and Midspan Acceleration Response of the Bridge}

As a basic check, it is necessary to determine whether it is possible to detect damage from the under-rear-wheel-spring acceleration response of the bus when a serious structural anomaly of a bridge has occurred. In other words, it is necessary to check whether the under-rear-wheel-spring response and the bridge response are coupled. This section looks at the correlation in terms of vibration properties during bridge crossing by using data obtained from another acceleration sensor installed to the "Shingondai Bridge" mentioned earlier.

Figure 17 shows an example of the relationship between the acceleration sensor location and the path of the bus. The conditions under which the bus actually crossed the bridge were as follows:

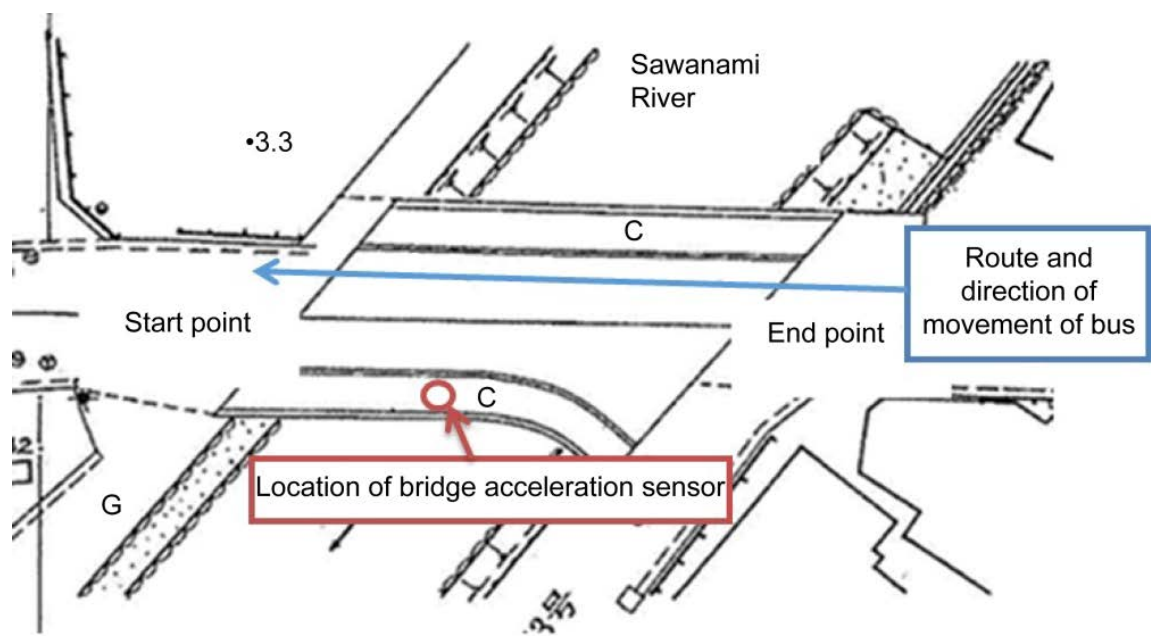

Figure 17. Acceleration sensor at the bridge and, the route and direction of movement of the bus. 
Weather: rain,

Vehicle speed: $35 \mathrm{~km} / \mathrm{h}$,

Number of oncoming vehicles: 1 ,

Total number of persons on the bus (including the bus driver): 10 .

Figure 18 shows an example of an acceleration waveform recorded when the bus was in the midspan zone of the bridge. As shown, the movement of the bus is coupled with the bridge vibration. The two acceleration response waveforms thus obtained from the measurement were analyzed by applying FSWT (Frequency Slice Wavelet Transform) [21] [22], which is a time-frequency space analysis technique, to determine whether there is time-series similarity in vibration properties between the vehicle and the bridge.

Figure 19(a) compares the acceleration response waveforms of the bus and the bridge recorded when the bus passed the midspan zone of the bridge. Figure 19(b) summarizes the FSWT analysis results. As shown, the response waveforms show similarity except at higher-order frequencies corresponding to external disturbances although the vertical axis (acceleration) of the acceleration response waveform needs to be adjusted by reducing the under-rear-wheel-spring response (see Figure 19(a)). As can be seen from the FSWT analysis results shown in Figure 19(b), the bridge underwent coupled vibration at around $12 \mathrm{~Hz}$ when the bus crossed the bridge. It has also been confirmed through measurement that the wheels under the springs of the bus were vibrating at around $12 \mathrm{~Hz}$ regardless of crossing the bridge or not [23]. From this, it can be concluded that the vibration properties of the bridge can be identified from the under-rear-wheelspring vibration of the bus by using the similarity between them.

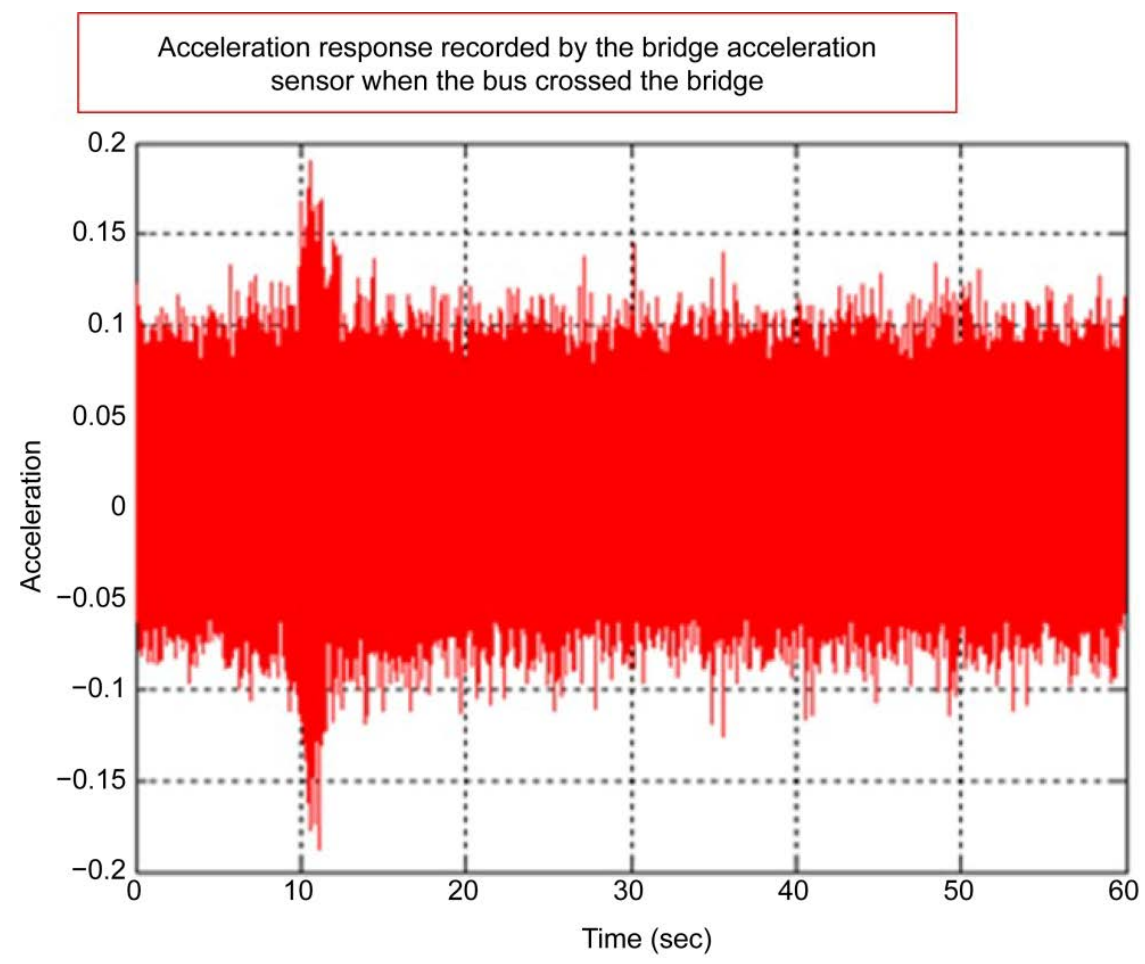

Figure 18. Example of acceleration response waveform in the midspan zone of the bridge. 


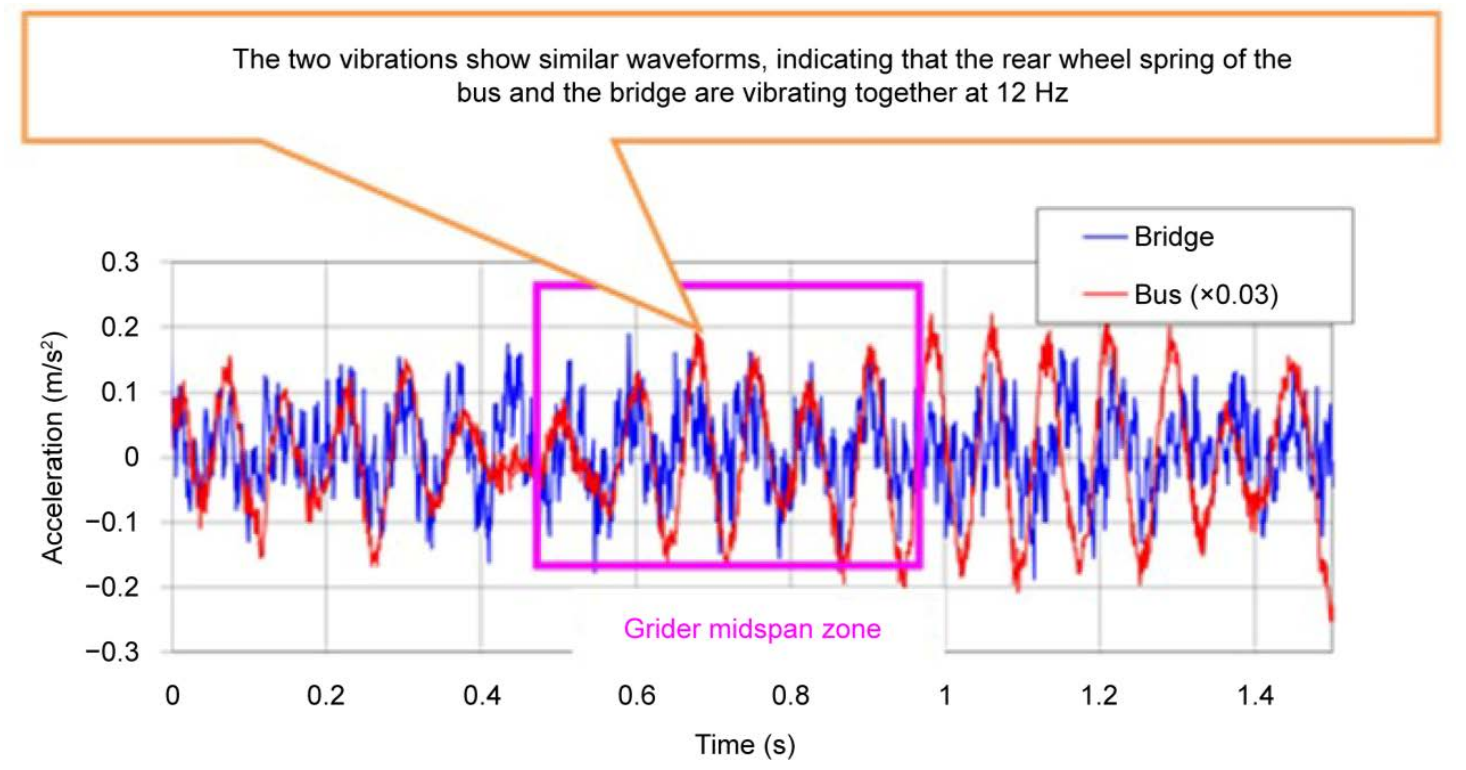

(a)
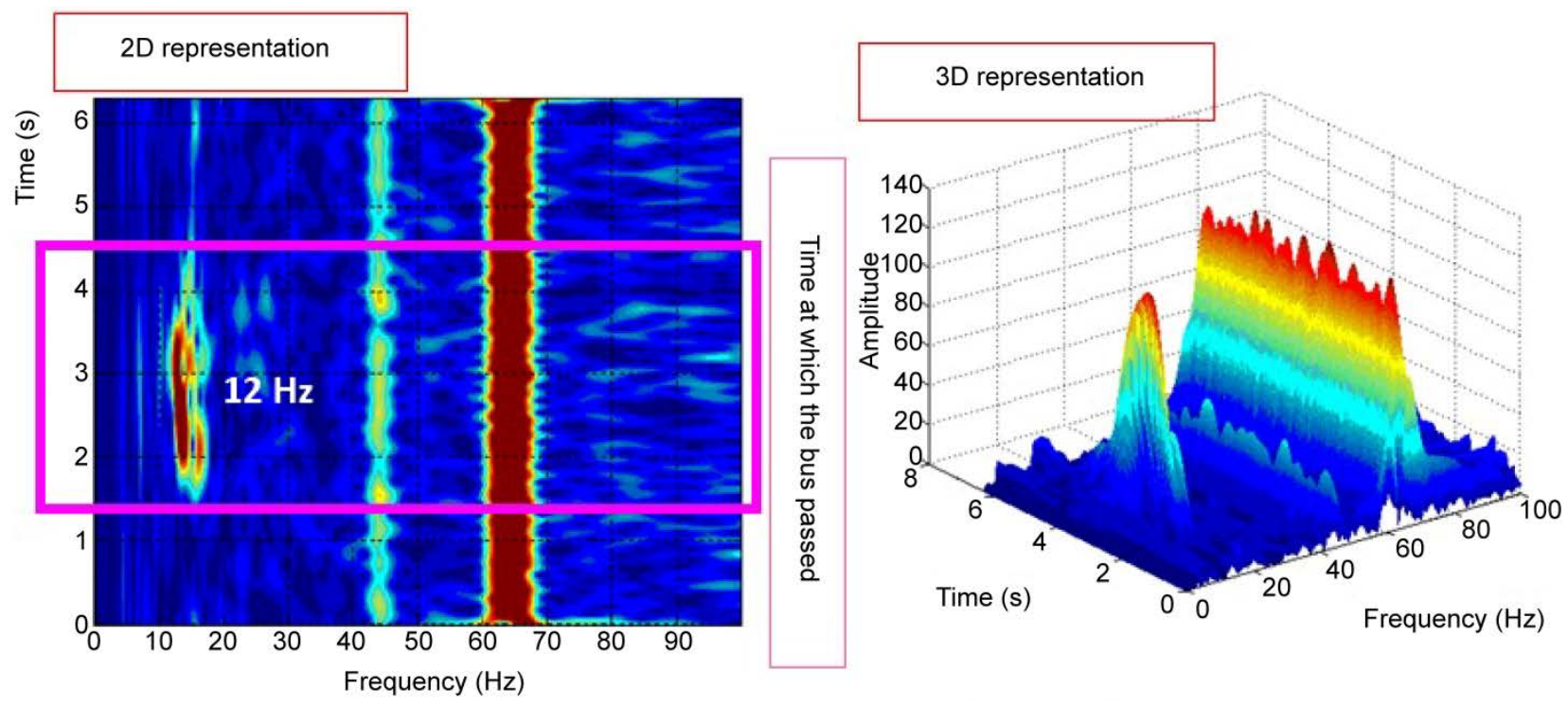

(b)

Figure 19. Comparison between midspan acceleration response and rear-wheel-under-spring acceleration response and, results of FSWT based time-frequency space analysis. (a) Comparison of girder-midspan and under-rear-wheel-spring acceleration responses; (b) Results of FSWT based time-frequency space analysis of bridge acceleration response waveform.

\subsubsection{Proposed Method of Characteristic Deflection Monitoring by Use of the Moving Average Method}

"Characteristic deflection" is affected by various external disturbances such as the bus operating conditions mentioned earlier. Consequently, "characteristic deflection" is inevitably subject to variation. An attempt was made, therefore, to determine changes over time in "characteristic deflection" obtained from the bus monitoring system by applying the moving average method, assuming that as the number of samples, $N$, increases, variations due to external disturbances such as bus operating conditions converge to a single value according to the central limit theorem. The moving average method is the method of calculating the av- 
erage of data in data section (segment; the number of data sets to be averaged) by calculating averages for incrementally shifted subsections. In the previous studies, the simple moving average method, which is one of the commonly used moving average methods, was used to process characteristic deflection data. As an example, Figure 20 shows the relationship between the number of data sections(segments) and the standard deviations of the corresponding "characteristic deflections" obtained by applying the moving average method to data subsets in the data section(segment).

As shown in Figure 20, as the number of data sets increases, the standard deviation becomes incrementally smaller. After the number of data sections reaches a certain level, the standard deviation does not change significantly and converges. This is thought to have shown that various external disturbances (error factors) can be characterized by standard deviations and averages if about 15 data sections are used, indicating that the central limit theorem mentioned earlier holds true. In fact, in the bus monitoring system, the standard deviation of characteristic deflection may be deemed to converge if the number of data sections is around 14 or 15. It was therefore decided to use 15 data sections in monitoring time-dependent changes in "characteristic deflection". On the basis of the concept described above, Figure 21 shows how characteristic deflection (measured value) and the simple moving average change. As shown in Figure 21 , measured values of "characteristic deflection" vary considerably, while simple moving averages of "characteristic deflection" are noticeably better in terms of variability.

\subsubsection{Development of Serious Deterioration (Damage) Criteria}

In the previous studies, characteristic deflection corresponding to the state of bridge damage determined in a vehicle-induced vibration simulation performed by the substructure method, a finite element method, was calculated. The intent was to develop serious deterioration (damage) criteria by which to identify the

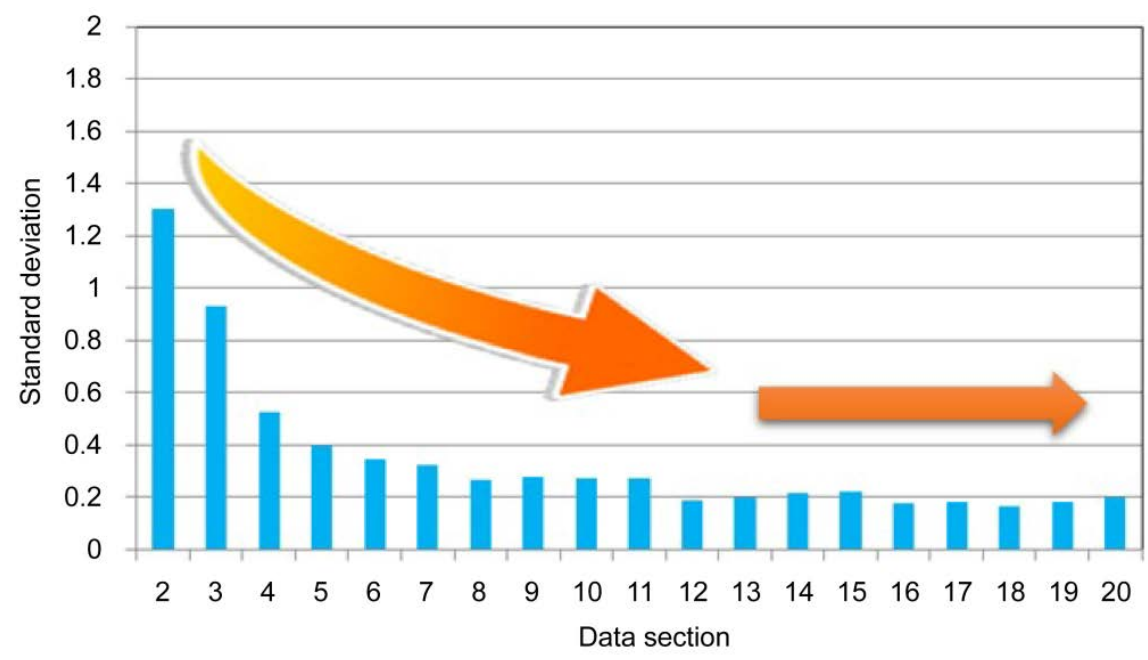

Figure 20. Example of differences in standard deviation of characteristic deflection among data sections. 
Changes in characteristic deflection (simple moving averages, 15 data sections)

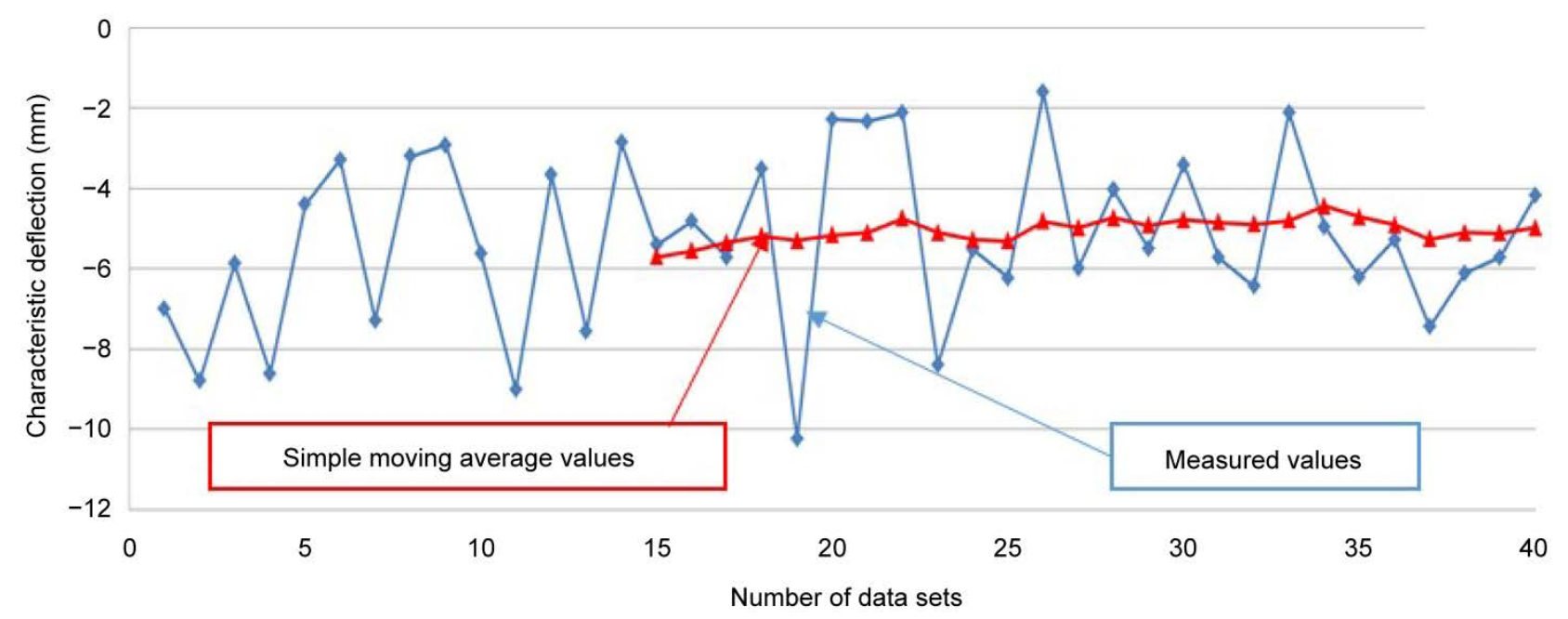

Figure 21. Examples of measured values of characteristic deflection and simple moving averages.

degree of change (increase) in characteristic deflection that indicates the occurrence of serious deterioration (damage) of the bridge of interest. In this study, in view of the fact that the bridge under consideration is a prestressed concrete girder bridge ("Shingondai Bridge", a single-span bridge built by the Bi-Prestressing Method) as mentioned earlier, attention is paid to the decrease in prestressing force as a kind of bridge damage. The National Institute for Land and Infrastructure Management (NILIM) of the Ministry of Land, Infrastructure, Transport and Tourism (MLIT) conducted a study on the relationship between the amount of prestress introduced and displacement (deflection) [24]. On the basis of that study, it has been shown through finite element analysis [25] that if a bridge is damaged so that the amount of prestressing force in a sound condition $(100 \%)$ decreases by $50 \%$, characteristic deflection increases by a factor of 1.93. It has also been shown that if the bridge is damaged so that the amount of prestressing force decreases by $90 \%$, characteristic deflection increases by a factor of 2.86 [25]. Table 4 summarizes the relationships of damage representations (sound, Deterioration Phase 1, Deterioration Phase 2) applicable to the "Shingondai Bridge" with the amount of decrease in prestressing force, the equivalent second moment of area and the amount of change in characteristic deflection. The calculated values obtained from the analysis of the seriously damaged bridge of interest (Shingondai Bridge) as mentioned earlier were used as serious deterioration (damage) criteria and compared with the measured changes (2010 to 2013) in characteristic deflection of the Shingondai Bridge. Examples of such comparisons are shown in Figure 22. For the purpose of comparison, the characteristic deflection obtained by multiplying the average of the first 15 measurements by 1.93 was used as the serious deterioration (damage) criterion (red line) for Deterioration Phase 1, and the characteristic deflection similarly obtained by multiplying by 2.86 was used as the serious deterioration (damage) criterion (green line) for Deterioration Phase 2. Thus, characteristic deflection is 
Table 4. Serious deterioration (damage) of "Shingondai Bridge (PC girder bridge)" used in vehicle-induced vibration simulation and changes in characteristic deflection.

\begin{tabular}{cccc}
\hline $\begin{array}{c}\text { Structural soundness } \\
\text { of bridge }\end{array}$ & $\begin{array}{c}\text { Decrease in } \\
\text { prestressing force }\end{array}$ & $\begin{array}{c}\text { Ratio of geometrical moment of } \\
\text { inertia relative to 0\% reduction }\end{array}$ & $\begin{array}{c}\text { Ratio of characteristic deflection } \\
\text { relative to 0\% reduction }\end{array}$ \\
\hline Sound & $0 \%$ & 1.0 & 1.0 \\
Deterioration Phase 1 & $50 \%$ & 0.52 & 1.93 \\
Deterioration Phase 2 & $90 \%$ & 0.35 & 2.86 \\
\hline
\end{tabular}

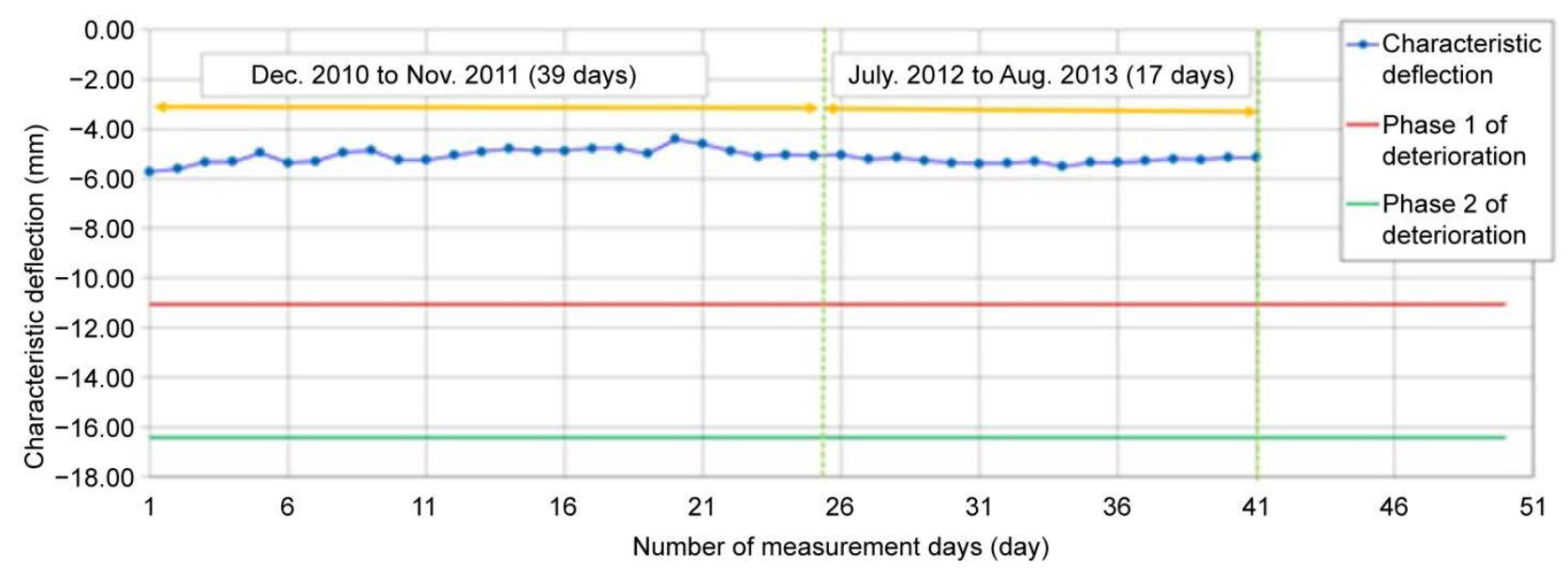

(a)

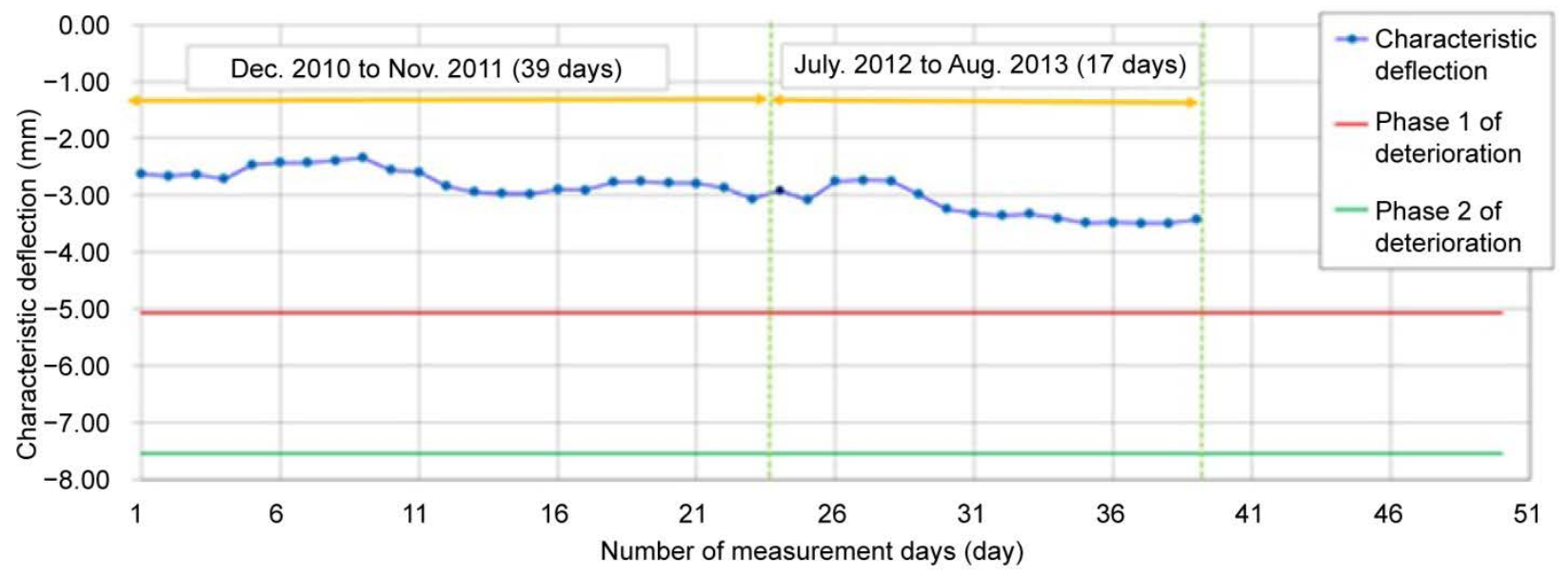

(b)

Figure 22. Example of changes in characteristic deflection from 2010 to 2013 (Shingondai Bridge) and comparison with the serious deterioration (damage) criteria(calculated values) shown in Table 4. (a) Tokonami $\rightarrow$ Nishikiwa Gakkomae; (b) Nishikiwa Gakkomae $\rightarrow$ Tokonami.

measured continually over a long period of time, and when one of those criterion values is reached, that is deemed to indicate the occurrence of some kind of serious damage in the bridge of interest, and a warning is issued so that necessary actions such as detailed inspection can be taken immediately. Actions such as detailed inspection need to be taken immediately when "characteristic deflection" has reached a criterion level on the out-bound or in-bound or in-bound route of the bus. 
As of this writing, the observation of the "characteristic deflection" of the "Shingondai Bridge" is underway while comparing the amount of decrease in prestressing force with the serious deterioration (damage) criteria. This approach, however, is not applicable to bridges of other types. For those bridges, it is necessary to identify a number of types of serious damage, taking account of such factors as the characteristics and material used of each bridge, and set serious damage criteria accordingly.

\subsection{Field-Test Findings Based on Four-Year Monitoring Data and Discussion}

On the basis of the basic findings of the previous studies mentioned in the preceding section, more measurement data have been accumulated (big data) by using the in-service municipal bus network of Ube-city, Japan. This section presents comprehensive verification results based on the monitoring data thus accumulated over a period of about four years. For that purpose, the influence of bus operating conditions (external disturbances) such as vehicle speed and the number of oncoming vehicles on changes in "characteristic deflection" (indicator used for damage detection) induced by in-service fixed-route bus operation is determined, and their correlations are reflected in conversion (correction) factors. All data on the three bridges that have been monitored by the "characteristic deflection" observation method proposed in the previous study accumulated over the four years are put together and examined to evaluate the usefulness of the proposed approach in detecting serious deterioration (damage) of the bridges being monitored.

\subsubsection{A Correlations between Bus Operating Conditions and Characteristic Deflection}

The bus monitoring system utilizes an in-service fixed-route bus. Its operating conditions, therefore, act as external disturbances during the long-term observation of "characteristic deflection". Because of this, as mentioned in Section 3.2.2, the amount of data accumulated in connection with the previous studies was not large enough to identify clear correlations between the bus operating conditions and "characteristic deflection". "Characteristic deflection" also varied considerably depending on the bridge concerned and the direction of vehicle movement. Consequently, the results obtained did not make it possible to reflect their correlations in conversion (correction) factors. An attempt is being made, therefore, to reduce the influence of external disturbance factors such as bus operating conditions by applying the simple moving average method. In this section, however, with the aim of evaluating the possibility of insufficiency of accumulated data, correlations between "characteristic deflection" and the bus operating conditions (external disturbances) are re-examined and discussed by putting together the four-year monitoring data again.

In this study, the coefficients of correlation between the "characteristic deflections" of the three bridges listed in Table 1 and the bus operating conditions (external disturbances) were calculated. The bus operating conditions that were 
taken into consideration as possible external disturbance factors included atmospheric temperature (Japan Meteorological Agency data) in addition to the factors considered in the previous studies, namely, weather (clear, rainy), the number of oncoming vehicles, the number of persons on the vehicle and vehicle speed. The data used for calculation were obtained by filtering the collected data according to certain criteria. Table 5 shows the calculation conditions (data filtering criteria) for each bridge. Table 6(a) and Table 6(b) summarize the correlations between the characteristic deflection of each bridge and the bus operating conditions calculated on the basis of the numbers of measurement data sets shown in Table 5. The correlation coefficient ranges and descriptions (definitions) of the correlations shown in Table 6 are as shown in Table 7.

As shown in Table 6, there is no bus operating condition that is strongly correlated with characteristic deflection, showing a coefficient of correlation exceeding 0.7. Turning attention to individual operating conditions, we notice that they show a positive correlation in some cases and a negative correlation in others, and it is difficult to conclude that any of those conditions shows a certain tendency. When calculating characteristic deflection, therefore, it is not possible to reflect correlations with those operating conditions in conversion (correction) factors by using the existing data alone.

In view of these results, it can be concluded that although it can be shown that each of the bus operating conditions (external disturbances) somehow influences the characteristic deflection, at present it is still not possible to quantify such influence so that it can be reflected in conversion (correction) factors. Therefore, as a method of handling variations due to various external disturbance factors including bus operating conditions, the moving average method (simple moving average method) mentioned in Section 3.2.2 was applied for the purpose of observing changes over time.

\subsubsection{Results of Observation of Characteristic Deflection in Previous Studies}

On the basis of the study results described in the preceding sections, this section deals with the calculated values of "characteristic deflection" based on the long-term monitoring of the three bridges in Ube-city's municipal bus network continued over a period of about four years, and the results of observation of changes over time in the characteristic deflection. Table 8 summarizes the measurement data, including the number of data sets, for the three bridges. Table 9

Table 5. Conditions (data restrictions) for correlation coefficient calculation by bridge.

\begin{tabular}{lccccc}
\hline \multicolumn{1}{c}{ Bridge name } & $\begin{array}{c}\text { Speed } \\
(\mathrm{km} / \mathrm{h})\end{array}$ & $\begin{array}{c}\text { Rainfall } \\
(\mathrm{mm})\end{array}$ & $\begin{array}{c}\text { Temperature } \\
\left({ }^{\circ} \mathrm{C}\right)\end{array}$ & $\begin{array}{c}\text { Oncoming traffic } \\
\text { (vehicles) }\end{array}$ & $\begin{array}{c}\text { Number of persons } \\
\text { on vehicle (persons) }\end{array}$ \\
\hline $\begin{array}{l}\text { Shingondai Bridge } \\
40-50\end{array}$ & 0 & $20-30$ & 0 & $5-15$ \\
$\begin{array}{l}\text { Shiratsuchi Daini } \\
\text { Bridge }\end{array}$ & $40-50$ & 0 & $20-30$ & 0 & $5-15$ \\
Jase Bridge & $45-55$ & 0 & $20-30$ & $0-1$ & No restriction \\
\hline
\end{tabular}


Table 6. (a) Correlations between characteristic deflection and bus operating conditions by bridge (correlation with vehicle speed/rainfall/temperature). (b) Correlations between characteristic deflection and bus operating conditions by bridge (correlation with number of oncoming vehicles/number of persons on bus).

(a)

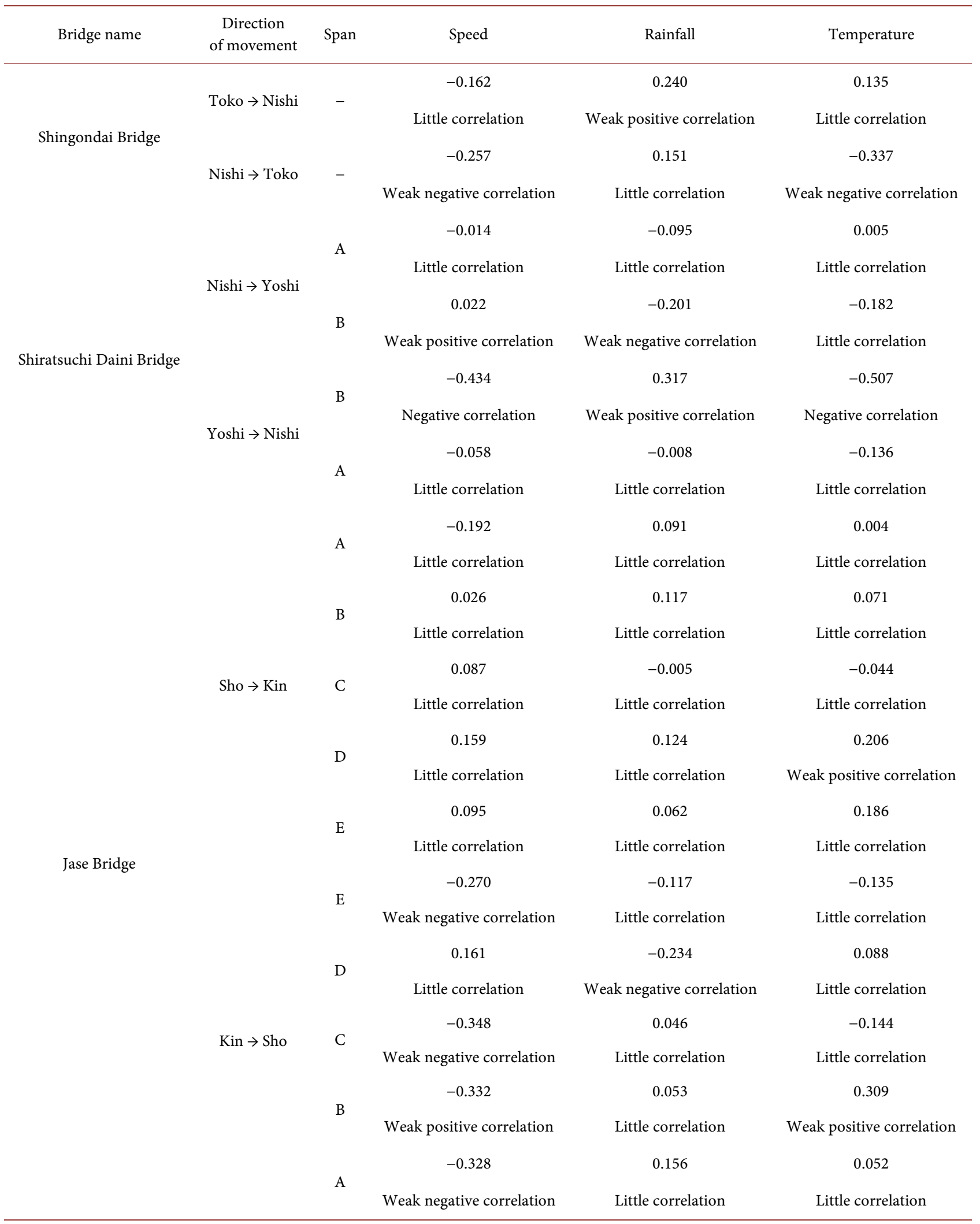


(b)

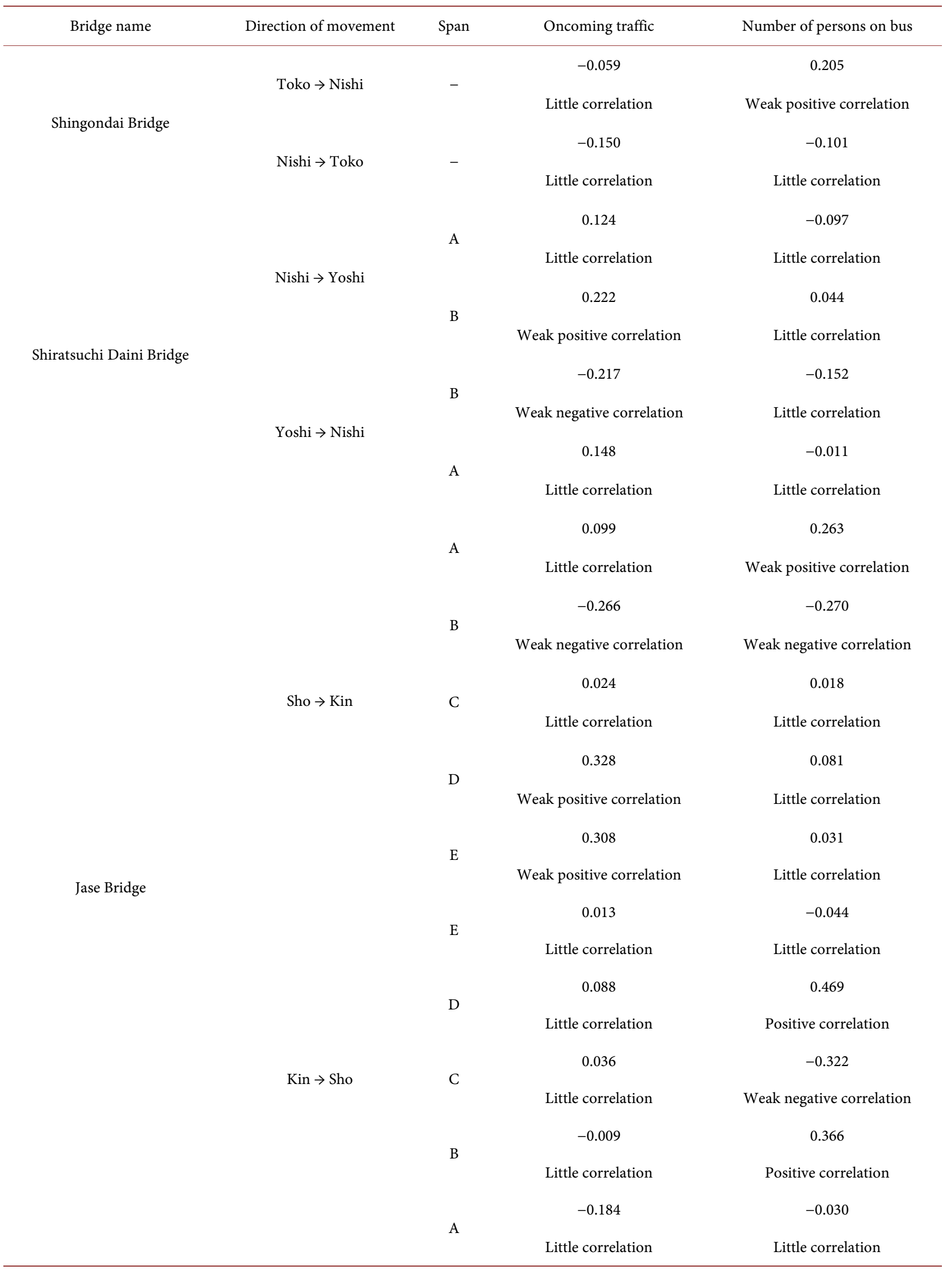


Table 7. Correspondence between the range of correlation coefficients and correlations in words.

\begin{tabular}{cc}
\hline Coefficient of correlation & Correlation \\
\hline $0.0- \pm 0.2$ & Little correlation \\
$\pm 0.2- \pm 0.4$ & Weak correlation \\
$\pm 0.4- \pm 0.7$ & Correlated \\
$\pm 0.7- \pm 0.9$ & Strong correlation \\
$\pm 0.9- \pm 1.0$ & Very strong correlation \\
\hline
\end{tabular}

Table 8. Number of measurement data sets.

\begin{tabular}{ccc}
\hline Bridge name & Direction of movement & Number of measurement data sets \\
\hline Shingondai Bridge & Toko $\rightarrow$ Nishi & 80 sets \\
Nishi $\rightarrow$ Toko & \\
Shiratsuchi Daini Bridge & Nishi $\rightarrow$ Yoshi & 77 sets \\
Yoshi $\rightarrow$ Nishi & 66 sets \\
Jase Bridge & Sho $\rightarrow$ Kin & 64 sets \\
\hline
\end{tabular}

Table 9. Calculated values of characteristic deflection by bridge/span.

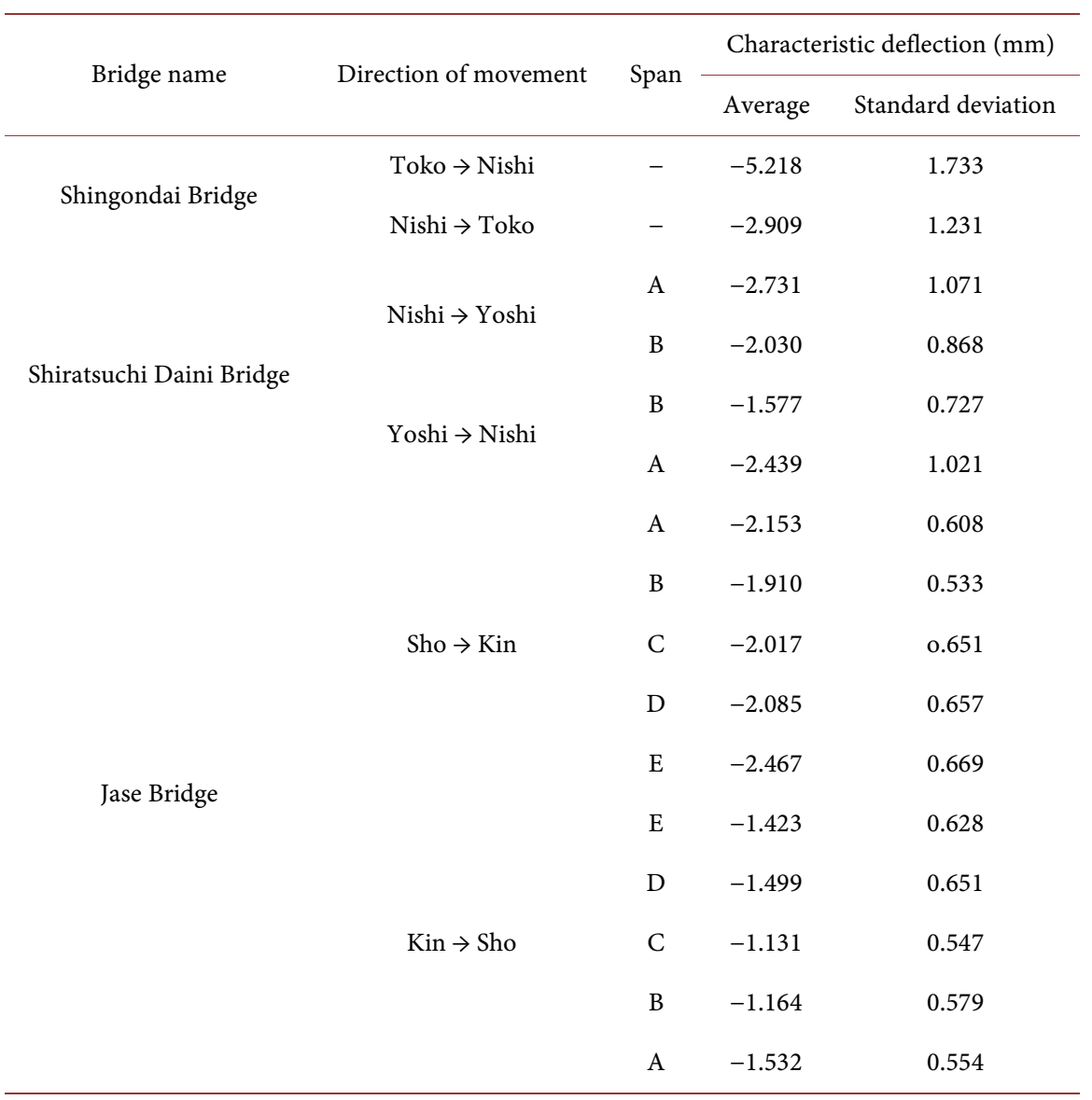


shows the span-by-span averages and standard deviations of "characteristic deflection" and other related data for the three bridges obtained by processing the four-year data in an integrated manner.

As shown in Table 9, characteristic deflection varies depending on the type of bridge, the direction of vehicle movement and span length. This is thought to be because of the bridge shapes in plan are asymmetric (e.g. curved bridge, skewed bridge, sidewalk on one side). As mentioned in Chapter 2, however, characteristic deflection is a quantity calculated by averaging estimated deflections in the same regions in each bridge or span. The differences mentioned above, therefore, do not pose any problem.

Figure 23 and Figure 24 show changes over time in the characteristic deflection of each span of the two bridges as an example, calculated by applying the simple moving average method mentioned earlier to the four-year monitoring data. As shown, characteristic deflection does not change sharply although it differs somewhat from span to span in the three bridges. It can therefore be concluded that at present the three bridges have not yet undergone serious deterioration (damage). Since, however, the deterioration (damage) of a short and medium span bridge tends to progress rapidly during the acceleration phase, it is necessary to continue long-term observation of changes in characteristic deflection.

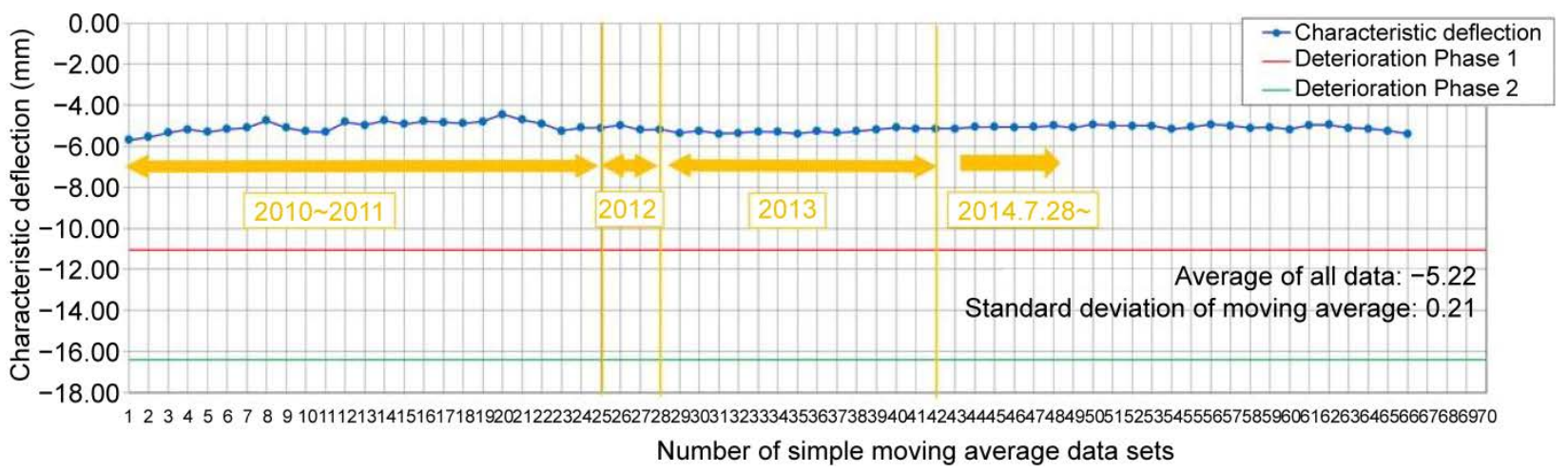

(a)

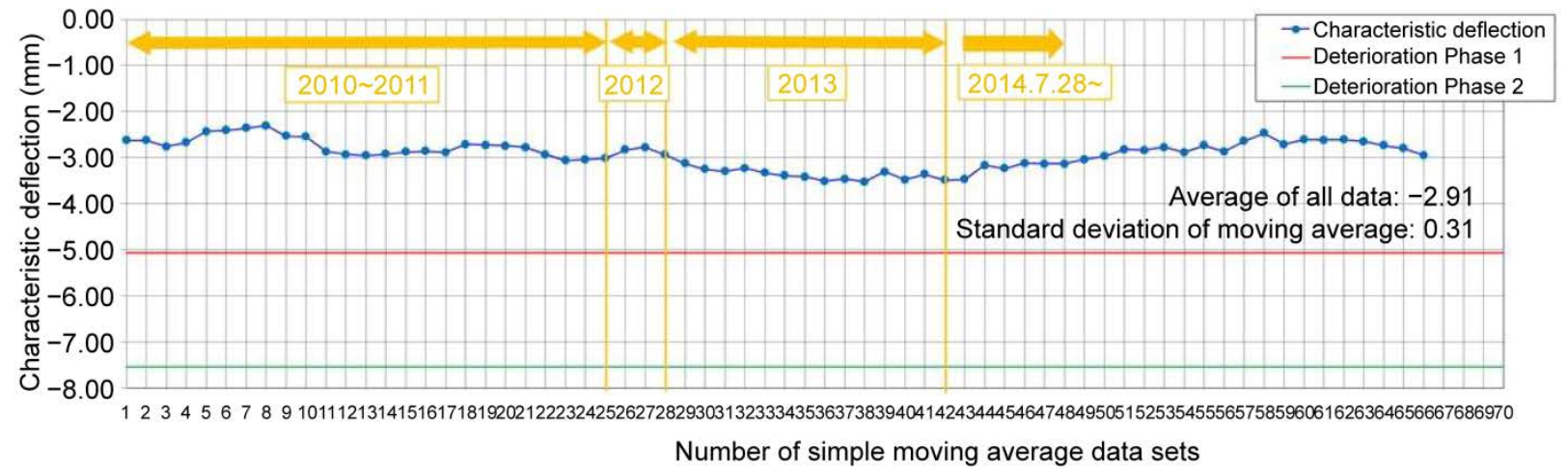

(b)

Figure 23. Characteristic deflection values obtained by applying the simple moving average method to four-year monitoring data (Shingondai Bridge). (a) Tokonami $\rightarrow$ Nishikiwa Gakkomae (Number of data sets: 80); (b) Nishikiwa Gakkomae $\rightarrow$ Tokonami (Number of data sets: 80 ). 


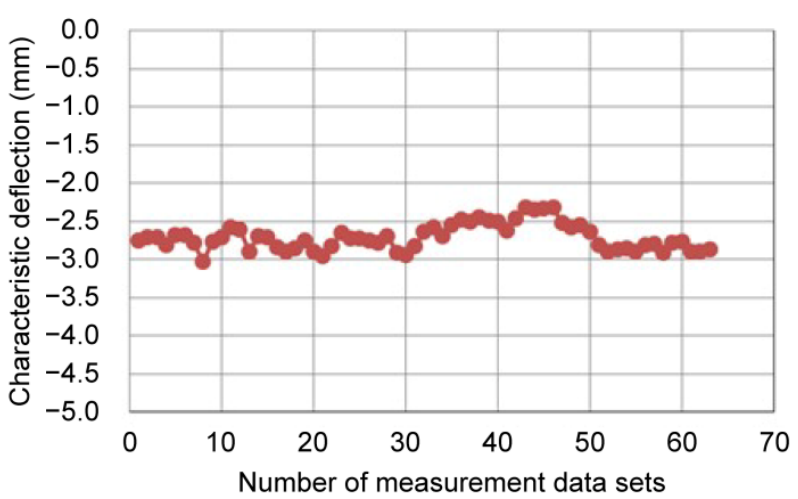

(a)

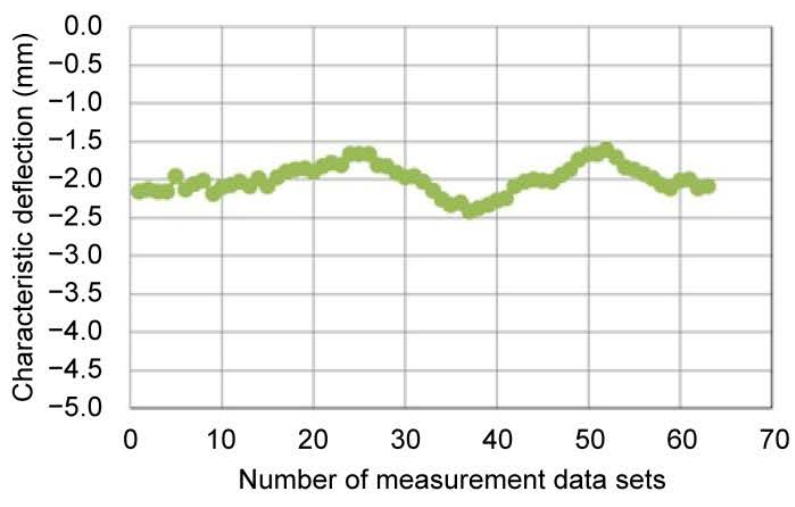

(c)

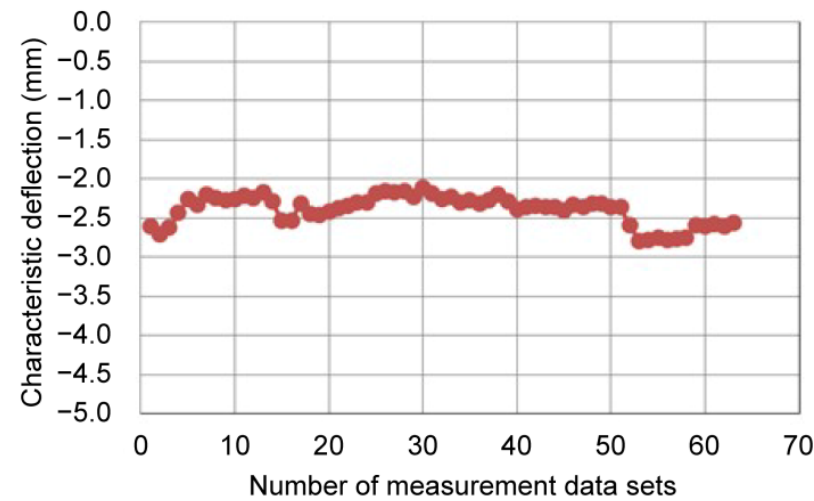

(b)

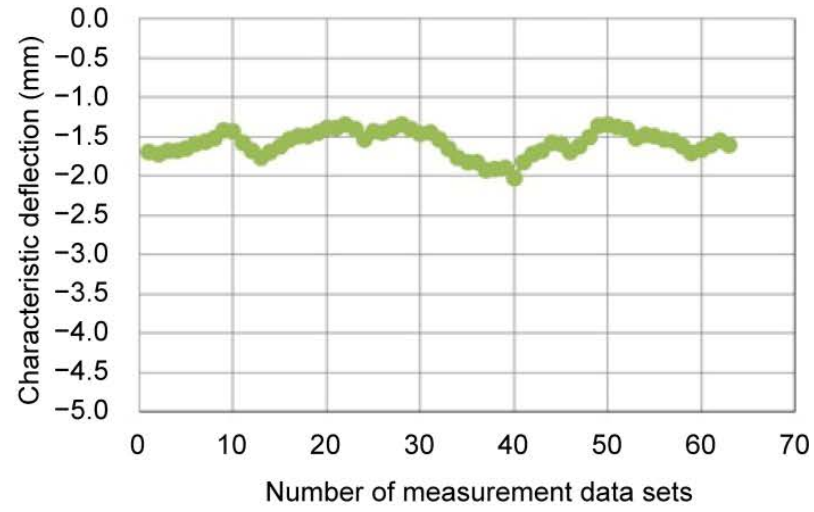

(d)

Figure 24. Characteristic deflection values obtained by applying the simple moving average method to four-year monitoring data (Shiratsuchi Daini Bridge). (a) Nishikiwa Gakkomae $\rightarrow$ Yoshida (Span A); (b) Yoshida $\rightarrow$ Nishikiwa Gakkomae (Span A); (c) Nishikiwa Gakkomae $\rightarrow$ Yoshida (Span B); (d) Yoshida $\rightarrow$ Nishikiwa Gakkomae (Span B).

\subsection{Summary and Future Tasks}

Thinking of bus operating conditions that may affect "characteristic deflection," which is an indicator of the structural health of bridges, as external disturbance factors, the authors tried to quantify the correlations between the bus operating conditions and the characteristic deflection by adding new measurement data to the available data. Although certain degrees of influence of external disturbance factors (bus operating conditions) can be seen, it is as yet not possible to quantify such influence in the absence of a clear tendency or a strong correlation. As a result, it was concluded that at present it is not possible to reflect their correlations in conversion (correction) factors applicable to the bus operating conditions. It was therefore thought that the simple moving average method mentioned in Section 3.2.2 would be useful in treating the influences of the external disturbance factors on the characteristic deflection as variances.

\section{Sensitivity Analysis of Damage Detection Using Moving Averages of Characteristic Deflection}

As mentioned in the preceding chapter, "characteristic deflection" measured with the bus monitoring system is affected by various external disturbance factors such as the operating conditions of the bus. Consequently, measured values 
vary significantly. In order to observe long-term changes, therefore, it has been proposed that measured values be processed by the moving average method [26] [27] [28] so that serious damage can be detected as early as possible. In the previous studies [9] [10], changes in "characteristic deflection" were observed over a long period of time by applying the simple moving average method. As mentioned in Section 3.4, however, the application of the simple moving average method raises concern about whether it is possible to detect, with sufficient sensitivity, when anomaly data indicative of serious bridge deterioration (damage) are newly input. In this chapter, therefore, results obtained by the simple moving average method are compared in detail with results obtained by other moving average methods (the weighted moving average method and the exponential moving average method). By so doing, the feasibility is evaluated of enhancing the sensitivity in detecting serious deterioration (damage) of short and medium span bridges.

\subsection{Overview and Characteristics of the Moving Average Method}

The moving average method is a method of analyzing data by smoothing out fluctuations of long-term time series data. The method, therefore, is widely used in various fields including engineering, finance and physical distribution. There are three widely used methods: the simple moving average method, the weighted moving average method and the exponential moving average method. Characteristics of the three moving average methods are described below.

\subsubsection{Simple Moving Average Method and Its Problems}

The simple moving average (SMA) method is the method of arithmetically averaging a subset of $N$ input data items without weighting. A simple moving average can be calculated as follows:

$$
S M A_{M}=\frac{p_{M}+p_{M-1}+p_{M-2}+\cdots+p_{M-N+1}}{N}
$$

where, $S M A_{M}$ is a simple moving average; $N$, the number of input data items; and $p$, the input value at each point in time.

An advantage of using Equation (25) is that if, for example, the simple moving average is to be calculated by using the input data obtained from the measurement carried out on the next day, calculation can be done by adding new input data and excluding the oldest input data as shown in Equation (26). In this method, therefore, there is no need to recalculate the sum.

$$
S M A_{M+1}=S M A+\frac{p_{M+1}}{N}-\frac{p_{M-N+1}}{N}
$$

This moving average method is the method used for the long-term observation of "characteristic deflection" in the previous studies [9] [10] mentioned earlier. Of the three moving average methods mentioned above, the simple moving average method enables simpler averaging of input time series data than in the other methods, but it also has its own problems. It is generally said that moving averages tend to lag behind (i.e. low sensitivity) the real time series input data 
and their trend. In the simple moving average method, newly added (input) values tend to be affected too greatly by past values deviating from the present average. This is why settings such as the number of data sections are important. In the calculation of "characteristic deflection" in the bus monitoring system in this study, the simple moving average method is applied to measured values of "characteristic deflection" to determine the relationship between their standard deviations and the number of data sections as shown in Figure 20 in Chapter 3. From the results thus obtained, it was concluded that standard deviations of characteristic deflection more or less converge when the number of data sections (segments) is 15 , and that should therefore be used for long-term observation.

In order to detect serious deterioration (damage) of the bridge of interest in using the bus monitoring system for bridge observation, it is necessary to detect points of abrupt change in "characteristic deflection" as soon as possible. In the conventional observation technique using the simple moving average method, however, the appearance of the influence of newly input data on characteristic deflection tends to lag behind because of averaging. This means that the detection of a point of abrupt change tends to be delayed (become less sensitive). It was therefore thought that weighting needs to be used in data processing so that detection sensitivity to newly input data can be improved.

\subsubsection{Weighted Moving Average Method and Exponential Moving Average Method}

Possible solutions to the abovementioned problem of damage detection sensitivity associated with the simple moving average method include the weighted moving average (WMA) method and the exponential moving average (EMA) method. Both of these methods assign weights to input data, but there are differences in the weighting scheme used and other details. Each method is described below.

\section{a) Weighted moving average method}

In the weighted moving average method, averages are calculated by assigning different weights to input values. To be more specific, the weighted moving average in the case where the number of data items is $N$ is calculated by assigning weight $N$ to the newest input value and weights $N-1, N-2, \cdots$ to the next newest values so that older values have smaller weights. The formula for calculating the weighted moving average is as follows:

$$
W M A_{M}=\frac{N p_{M}+(N-1) p_{M-1}+\cdots+2 p_{M-N+2}+p_{M-N+1}}{N+(N-1)+\cdots+2+1}
$$

where, $W M A_{M}$ is the weighted moving average; $N$, the number of input data items and weight; and $p$, input data at each point in time.

From Equation (27), we obtain

$$
W M A_{M+1}=\frac{N p_{M+1}+(N-1) p_{M}+\cdots+2 p_{M-N+3}+p_{M-N+2}}{N+(N-1)+\cdots+2+1}
$$

From Equation (27) and Equation (28), the difference between the numerators 
of $W M A_{M+1}$ and $W M A_{M}$ can be expressed as,

$$
\text { Numerator }_{M+1}-\text { Numerator }_{M}=N p_{M+1}-p_{M}-p_{M-1}-\cdots-p_{M-N+2}-p_{M-N+1}
$$

For example, let $\operatorname{Total}_{M}$ represent the sum of the input values entered during a period of $N$ days. Then, Total $_{M}$ can be calculated as,

$$
\operatorname{Total}_{M}=p_{M}+p_{M-1}+\cdots+p_{M-N+1}
$$

From Equation (29) and Equation (30)

$$
\text { Numerator }_{M+1}=\text { Numerator }_{M}+N p_{M-1}-\text { Total }_{M}
$$

Hence,

$$
W M A_{M+1}=\frac{\text { Numerator }_{M+1}}{N+(N-1)+\cdots+2+1}
$$

It can be seen from Equation (32) derived in the last step that as with simple moving averages, there is no need to recalculate the sum when calculating the weighted moving average reflecting the measurement data entered on the next day.

\section{b) Exponential moving average method}

Like the weighted moving average method, the exponential moving average method is a moving average calculation method that assigns different weights to individual input values. The difference is that in the exponential moving average method, the largest weight is assigned to the newest input value, but weights assigned to older input values are reduced exponentially. By so doing, greater importance is attached to newer input data, but older input data are not discarded altogether. In other words, the weight assigned to the oldest input value is not zero.

The degree of weight reduction is determined by the smoothing coefficient, $\alpha$, which takes values between 0 and 1 . Generally, $\alpha$ is defined, by using the number of input data items, $N$, as $\alpha=\frac{2}{N+1}$. The formula for calculating the exponential moving average is as follows:

$$
E M A_{M}=E M A_{M-1}+\alpha \times\left(p_{M}-E M A_{M-1}\right)
$$

where, $E M A_{M}$ is the exponential moving average; $\alpha$, the smoothing coefficient; and $p$, the input data value at each point in time.

Expanding $E M A_{M-1}$ in Equation (33) gives,

$$
E M A_{M}=\alpha \times\left\{p_{M}+(1-\alpha) p_{M-1}+(1-\alpha)^{2} p_{M-2}+\cdots\right\}
$$

As shown in Equation (34), weights assigned to input values decrease exponentially from the weight assigned to the newest input value. In Equation (34), which expresses a sum, the value $(1-\alpha)$ is smaller than 1 . Higher-order terms, therefore, become smaller, and terms of or higher than a certain order are negligibly small.

Expanding Equation (33) by using $\alpha=\frac{2}{N+1}$ gives, 


$$
E M A_{M}=\frac{(N-1) E M A_{M-1}+2 p_{M}}{N+1}
$$

Equation (35) seems to indicate that only newly input data are heavily weighted and the other data are given the same weight. The fact, however, is that when a new measured value is input, it is incorporated into the previous average, and this process is repeated so that the weight assigned to the newly input value gradually decreases.

\subsection{Comparison of Different Moving Average Methods in Sensitivity to Changes Incharacteristic Deflection}

By using the three moving average methods mentioned above, this section performs sensitivity analyses of the cases where abnormal values obtained from long-term observation of changes in "characteristic deflection" are input. Focusing on the cases where abnormal values of change in the "characteristic deflection" of the "Shingondai Bridge", one of the three bridges under consideration in this study, designed to simulate serious damage are input consecutively, the analyses compare and evaluate the following: (1) the number of inputs needed to reach the serious deterioration (damage) criterion level ("Deterioration Phase 1" defined in Section 3.2.3) and (2) the slope of the curve showing the average values obtained by each moving average method when values indicative of an anomaly are input consecutively. On the basis of the results thus obtained, an attempt is made to enhance the "characteristic deflection" observation accuracy and damage detection sensitivity of the bus monitoring system in monitoring short and medium span bridges over a long period of time.

\subsubsection{Comparison in the Number of Inputs Needed to Reach the Deterioration Phase 1 Level When Given Anomaly Data}

As mentioned in Section 3.2.3, serious deterioration (damage) criteria based on the amount of decrease in prestressing force were set for the evaluation of the "characteristic deflection" of the "Shingondai Bridge" (prestressed concrete girder bridge). In this section, detection sensitivity attainable by the different moving average methods is compared in terms of the number of inputs needed to reach the Deterioration Phase 1 level in the cases where anomaly data attributable to serious deterioration (damage) are input consecutively. The analyses consider a total of four cases: the cases where constant values equal to three, four and five times, respectively, the average value of "characteristic deflection" calculated through the bus-based monitoring of the present condition of the "Shingondai Bridge" are input consecutively and the case where the input value is gradually increased to two, three, four, ...n times the average value of "characteristic deflection" (to simulate the case where deterioration or damage of the bridge under consideration gradually increases). The anomaly data to be used as inputs in these cases, which are referred to as Case 1, Case 2, Case 3 and Case 4, respectively, are shown in Table 10.

As an example, Figure 25 graphically compares the changes in "characteristic deflection" calculated through the bus-based monitoring of the "Shingondai 
Table 10. Anomaly data used as inputs.

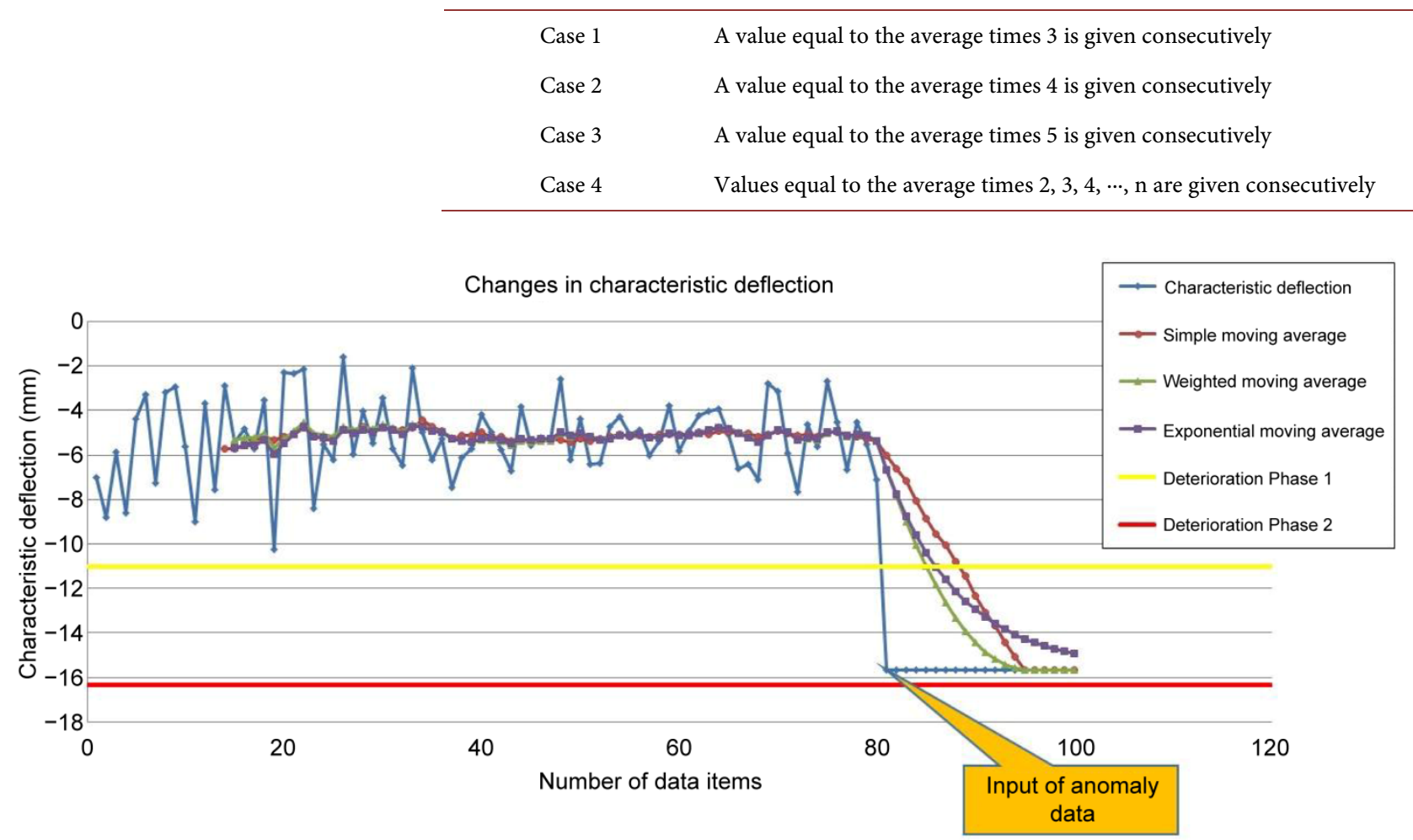

Figure 25. Examples of different characteristic deflection curves obtained by different moving average methods when Case 1 anomaly data are input consecutively.

Bridge" with the changes of the curve obtained by each moving average method in the case where Case 1 anomaly data are input consecutively. In order to quantify the detection sensitivity in the cases where Case 1 to Case 4 data on anomalies attributable to serious deterioration (damage) of the bridge of interest are input consecutively, the number of inputs needed by the curve obtained by each moving average method to reach the serious deterioration (damage) criterion level when Case 1 to Case 4 anomaly data are input consecutively was determined. Table 11 shows the numbers of inputs thus determined. In each case, the smallest number of inputs needed in each moving average method to reach the Deterioration Phase 1 level is shown with yellow shading (the Deterioration Phase 2 results are also shown for reference only).

From Table 11, it can be seen that in all cases the serious deterioration (damage) criterion value is reached with a smaller number of inputs (i.e. higher sensitivity) when either the weighted moving average method or both the weighted moving average method and the exponential moving average method are used. It has also been found, however, that in all moving average methods, a certain number of inputs are required (detection lags behind) before the serious deterioration (damage) criterion value, which is an indicator by which to detect a change in "characteristic deflection", is reached. The goal of damage detection by means of the bus monitoring system is to detect serious deterioration of the bridge of interest as early as possible. The study findings described above, 
Table 11. Comparison of the number of inputs needed to reach the serious deterioration (damage) criterion level when anomaly data are input consecutively.

\begin{tabular}{|c|c|c|c|c|c|}
\hline Number of inputs & Case & Case 1 & Case 2 & Case 3 & Case 4 \\
\hline \multirow{3}{*}{$\begin{array}{c}\text { Number of inputs } \\
\text { needed to reach } \\
\text { Deterioration Phase } 1\end{array}$} & $\begin{array}{l}\text { Simple moving } \\
\text { average method }\end{array}$ & 9 & 6 & 5 & 6 \\
\hline & $\begin{array}{l}\text { Weighted moving } \\
\text { average method }\end{array}$ & 6 & 4 & 3 & 4 \\
\hline & $\begin{array}{l}\text { Exponential moving } \\
\text { average method }\end{array}$ & 6 & 4 & 3 & 4 \\
\hline \multirow{3}{*}{$\begin{array}{c}\text { Number of inputs } \\
\text { needed to reach } \\
\text { Deterioration Phase } 2\end{array}$} & $\begin{array}{l}\text { Simple moving } \\
\text { average method }\end{array}$ & & 11 & 8 & 8 \\
\hline & $\begin{array}{l}\text { Weighted moving } \\
\text { average method }\end{array}$ & & 8 & 5 & 6 \\
\hline & $\begin{array}{l}\text { Exponential moving } \\
\text { average method }\end{array}$ & & 10 & 6 & 6 \\
\hline
\end{tabular}

however, indicate that in the long-term observation of "characteristic deflection", the detection of serious deterioration (damage) of the bridge of interest might lag behind (i.e. lower sensitivity). In the next section, therefore, attention is turned to the slope of the "characteristic deflection" curve at a point of abrupt change after the second input of anomaly data as another possible indicator for damage detection, and detection sensitivity attainable by using that indicator is compared and evaluated.

\subsubsection{Comparison of the Slopes of Moving Average Curves Corresponding to Anomaly Data}

In order to solve the above mentioned problem, it needs to find out an indicator capable of capturing a change in the characteristic deflection curve immediately after anomaly data are input to the system. Attention is turned, therefore, to the slope of the curve. To be more specific, a comparison is made focusing on the slope of the moving average curve after predetermined anomaly data are input two times consecutively. The reason why attention is turned to the curve after two consecutive inputs of anomaly data is as follows. As mentioned earlier, "characteristic deflection" varies considerably because of external disturbance factors. Consequently, it is not uncommon that calculated values of "characteristic deflection" change sharply from the previous input data. The aim, therefore, is to establish criteria by which to detect and evaluate (possible) serious deterioration (damage) if an abrupt change in characteristic deflection has been indicated by calculation two times consecutively. The anomaly data used here is the Deterioration Phase 1 value mentioned as an example in Section 3.2.3. To consider a wide range of conditions, the timing of the consecutive input of anomaly data was determined as follows. Of the characteristic deflection values calculated by the moving average method at or after the 15th input counted from the first input data from which "characteristic deflection" begins to change, a total of 10 points consisting of the five largest values and the five smallest values are se- 
lected. Then, the slopes of the curves obtained by inputting the anomaly data two times consecutively at each point are compared. Examples of the 10 points thus selected are shown with red circles (1) to (10) in Figure 26. Table 12 shows the average, standard deviation and variance obtained from the entire data and those obtained by each moving average method.

Table 13 shows the calculated slopes of the curves obtained by the different moving average methods in the cases where the anomaly data are input two times consecutively at the 10 points (1) to (10) selected as described above (the figures in the shaded areas are the largest and smallest slope values obtained by each moving average method). Table 14 shows the values obtained by subtracting the minimum slope (simple: -0.2753 , weighted: -0.2545 , exponential: -0.2512 ) in the case where the anomaly data are not input two times consecutively from the maximum slope (shaded in red) and minimum slope (shaded in blue) of the curve obtained by each moving average method.

\subsection{Discussion and Summary}

As indicated by the results shown in Table 14, the value obtained by subtracting the non-anomaly-data slope from the slope of the "characteristic deflection" curve obtained by inputting the anomaly data two times consecutively (difference: indicator for damage detection) tends to be slightly smaller than the minimum value of the slope obtained by the simple moving average method (shaded in blue). This means that serious deterioration (damage) in a case like this may be overlooked if the simple moving average method is used. In contrast, when

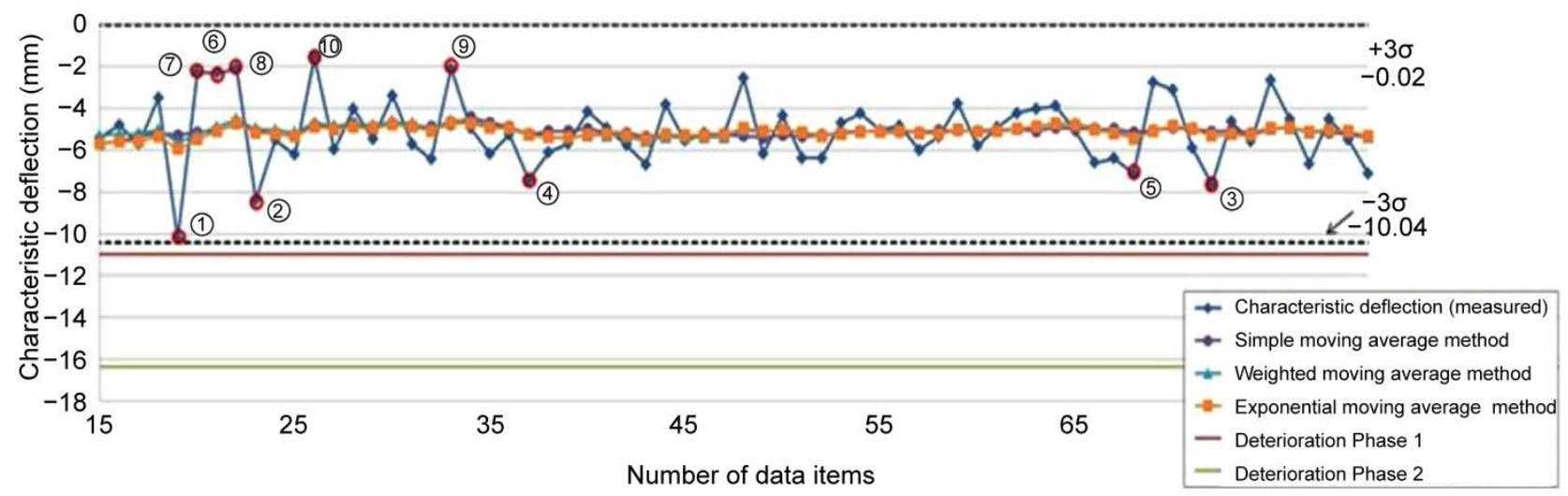

Figure 26. Timing of consecutive inputs of anomaly data (Points (1) to (10)).

Table 12. Statistical quantities of characteristic deflection obtained from all data by different moving average methods.

\begin{tabular}{|c|c|c|c|c|}
\hline & Statistical quantity & Average $(\mathrm{mm})$ & Standard deviation $(\mathrm{mm})$ & Variance \\
\hline & All data & -5.218 & 1.733 & 3.004 \\
\hline \multirow{3}{*}{$\begin{array}{l}15 \text { data } \\
\text { sections }\end{array}$} & Simple moving average method & -5.108 & 0.212 & 0.045 \\
\hline & Weighted moving average method & -5.117 & 0.310 & 0.096 \\
\hline & Exponential moving average method & -5.174 & 0.306 & 0.094 \\
\hline
\end{tabular}


Table 13. Comparison of the slopes of characteristic deflection curves obtained by different moving average methods by inputting anomaly data two times consecutively $(\mathrm{mm} /$ input).

\begin{tabular}{cccc}
$\begin{array}{c}\text { Moving average } \\
\text { method }\end{array}$ & $\begin{array}{c}\text { Simple moving } \\
\text { average method }\end{array}$ & $\begin{array}{c}\text { Weighted moving } \\
\text { average method }\end{array}$ & $\begin{array}{c}\text { Exponential moving } \\
\text { average method }\end{array}$ \\
$\begin{array}{c}\text { Timing of anomaly } \\
\text { data input }\end{array}$ & -0.4770 & -0.6850 & -0.5924 \\
\hline (1) & -0.4486 & -0.7041 & -0.6820 \\
(2) & -0.4278 & -0.7117 & -0.6650 \\
(4) & -0.2693 & -0.7074 & -0.6705 \\
(5) & -0.4223 & -0.7012 & -0.6514 \\
(6) & -0.3840 & -0.7226 & -0.6923 \\
(7) & -0.3810 & -0.6980 & -0.6461 \\
(8) & -0.5293 & -0.7486 & -0.7359 \\
(9) & -0.3160 & -0.7725 & -0.7365 \\
(10) & -0.3593 & -0.7423 & -0.7172 \\
\hline
\end{tabular}

*See Figure 26.

Table 14. Maximum and minimum differences in the slope of characteristic deflection curve: with vs. without anomaly data input ( $\mathrm{mm} /$ input).

\begin{tabular}{lcc}
\hline $\begin{array}{l}\text { Moving average } \\
\text { method }\end{array}$ & Difference & Maximum difference \\
\hline Simple moving average method & 0.0060 & -0.2540 \\
Weighted moving average method & -0.4305 & -0.4941 \\
Exponential moving average method & -0.3412 & -0.4853 \\
\hline
\end{tabular}

the weighted moving average method or the exponential moving average method is used, the slope of the curve tends to change noticeably when the anomaly data are input two times consecutively (see Table 14). The weighted moving average method tends to show a larger slope than the exponential moving average method in response to the input of the anomaly data. Thus, it has been shown that among the three moving average methods experimentally applied in this study, the weighted moving average method is most sensitive to anomaly data input. Judging comprehensively, therefore, from the results related to the number of anomaly data inputs needed to reach the serious deterioration (damage) criterion level and the slope of the curve obtained by inputting the anomaly data, it can be concluded that the moving average method most suitable for serious deterioration (damage) detection in the long-term observation of "characteristic deflection" is the weighted moving average method.

As pointed out in Section 4.2.1, the method of damage detection relying solely on the number of inputs needed to reach the serious deterioration (damage) criterion level can result in a delay in taking response action. Using that method in 
conjunction with the method of using a newly employed damage indicator, namely, the slope of the "characteristic deflection" curve, will help improve the sensitivity in detecting serious bridge damage. In order to put that approach to practical use, it is necessary to establish rational serious deterioration (damage) criteria associated with the slope of the characteristic deflection curve.

\section{Damage Detection Sensitivity of Artificial Damage on Characteristic Deflection}

To evaluate the sensitivity of "characteristic deflection" used by the bus monitoring system as a serious damage indicator, the influence of artificial damage (bridge guardrail removal) on "characteristic deflection" was evaluated by using a decommissioned bridge (a real old bridge to be removed shortly). This section deals with the field test results thus obtained. A simulation analysis using an analysis model allowing for the coupling between the bus and the bridge is also performed to calculate changes in the "characteristic deflection" of the artificially damaged bridge, and damage detection sensitivity is compared and evaluated from the analytical point of view.

\subsection{Overview of the Test}

The field test was carried out by using a 72-year-old (as of the time of the study) reinforced concrete bridge to be dismantled and removed shortly ("Sakae Bridge" [29]; see Figure 27). In view of factors such as seasonal (temperature) changes and the presence or absence of bridge guardrails, it was decided to conduct measurement using the bus monitoring system a total of four times, namely, in September 2012 and in January, February and March 2013. As in the case of the long-term field test mentioned earlier, vertical (z-axis) acceleration response and "characteristic deflection" occurring when the municipal bus (vehicle) running at a constant speed crosses the bridge in the in-bound and

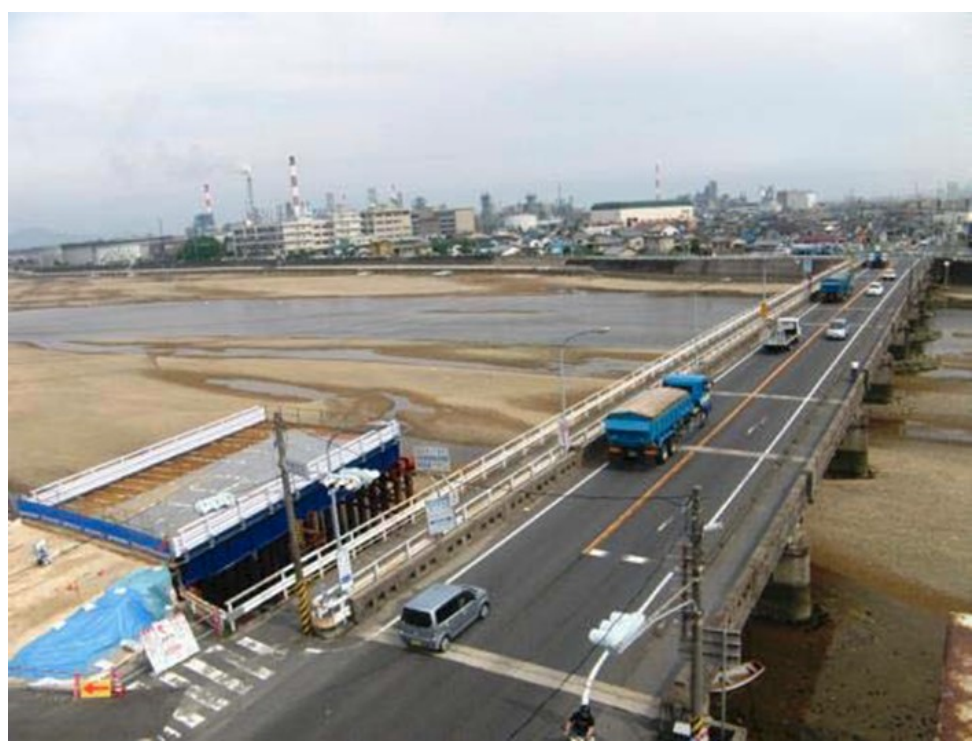

Figure 27. General view of the field test bridge (Sakae Bridge) when in service. 
out-bound lanes (15 in-bound runs and 15 out-bound runs) are calculated. Taking advantage of the absence of other road traffic (ideal condition) because the bridge was already in the process of demotion and removal, the measurements were conducted under various conditions by varying such conditions as vehicle weight and vehicle speed (constant speed).

\subsubsection{Types and Characteristics of Vehicles Used for Measurement}

The vehicles used for the measurement purpose are Ube-city's sightseeing bus having a gross weight of about $15 \mathrm{tf}$ and a mini vehicle having a gross weight of about $1.3 \mathrm{tf}$. As in the long-term field test mentioned earlier, an acceleration sensor was installed at a similar position (under the rear wheel spring) of each vehicle for continuous measurement. Besides this sensor, three other acceleration sensors of comparable performance were also installed [on the right side, at the center (normal location) and on the left side under the rear wheel spring] to evaluate the influence of sensor locations. Figure 28(a) and Figure 28(b) shows general views of the two types of vehicles used and the acceleration sensor locations. Table 15(a) and Table 15(b) show the specifications of the vehicles used and data on the performance and other details of the sensors.

\subsubsection{Overview of the Bridge}

The "Sakae Bridge" used for the field measurement is a 168.3-meter-long, 11.0meter-wide eight-span simple cantilever reinforced-concrete T-girder bridge completed in 1941 (managed by the Ministry of Land, Infrastructure, Transport and Tourism). For reconstruction, the bridge was demolished and removed by stages over a period of two years from fiscal year 2012. Table 16 and Figure 29 show the structural specifications and pre-removal configuration and dimensions of the Sakae Bridge and the bridge section where the field test was conducted. Since there was no other road traffic on the bridge at the time of measurement, only seasonal (temperature) changes were taken into consideration as an external disturbance factor affecting characteristic deflection in evaluating the influence of the presence or absence of bridge guardrails (regarded as artificial damage). For the purpose of that evaluation, a field test was carried out on four occasions (once in autumn, twice in winter and once in spring). For the evaluation of changes in characteristic deflection before and after bridge guardrail removal, the data obtained in January and February 2013 were compared. Figure 30 shows the bridge before and after the guardrail removal.

\subsection{Test Results and Discussion}

This section puts together and discusses the results obtained from the field test conducted by using the decommissioned bridge as mentioned above.

\subsubsection{Differences in Sensor Location}

Changes in characteristic deflection depending on the location of the acceleration sensor installed under the rear wheel spring were examined. Figure $31 \mathrm{com}-$ pares the acceleration response waveforms obtained from the different sensor 

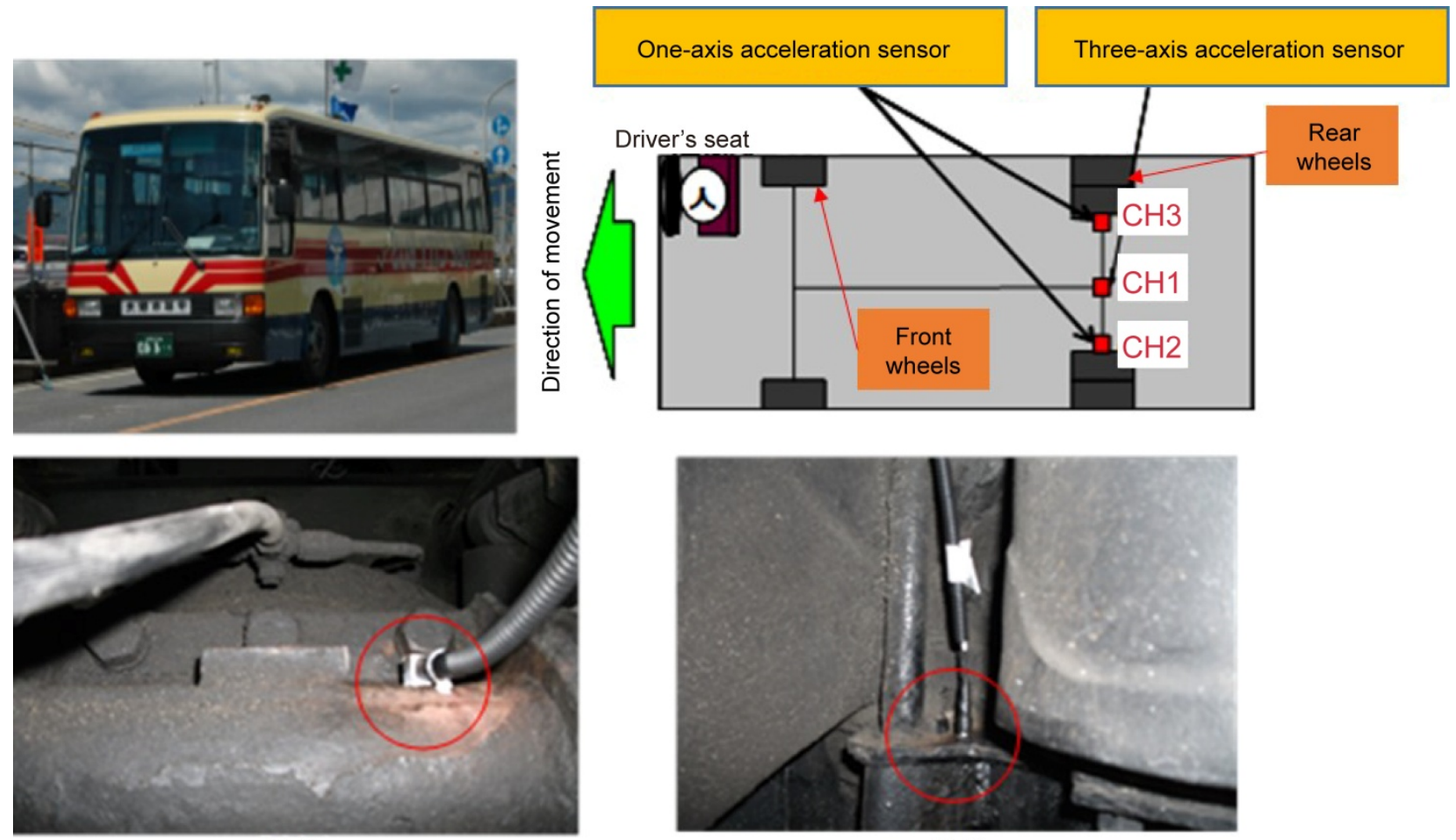

$\mathrm{CH} 1$

$\mathrm{CH} 3$

(a)
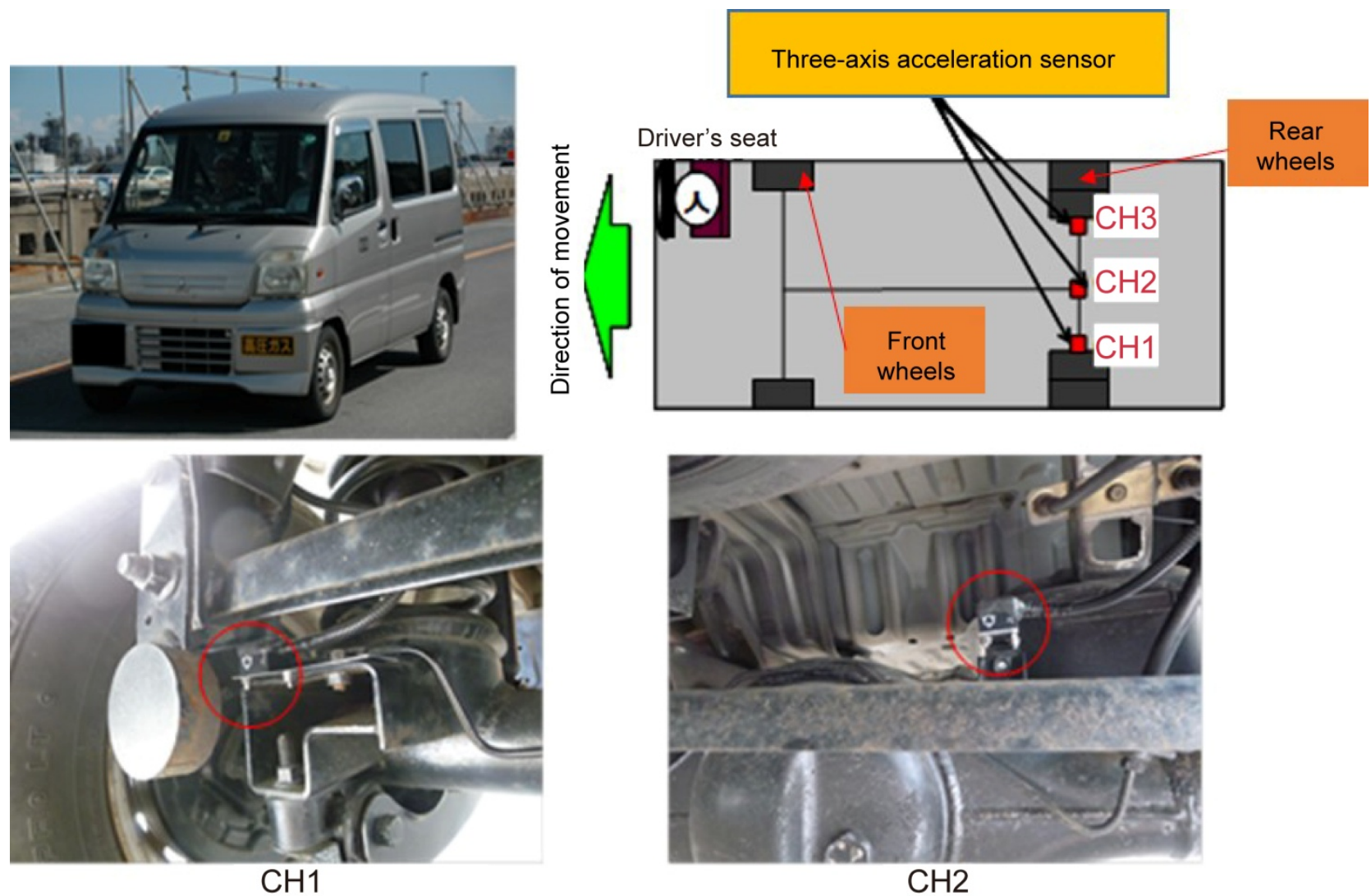

(b)

Figure 28. Measurement vehicles and acceleration sensor locations (Sightseeing bus \& Mini vehicle). (a) Sightseeing bus; (b) Mini vehicle. 
Table 15. Specifications of measurement vehicles and acceleration sensors. (a) Measurement vehicles; (b) Acceleration sensors.

(a)

\begin{tabular}{ccc}
\hline Items & Bus & Mini vehicle \\
\hline Riding capacity & 57 persons & $2(4)$ \\
Length & $1,194 \mathrm{~cm}$ & $339 \mathrm{~cm}$ \\
Width & $249 \mathrm{~cm}$ & $147 \mathrm{~cm}$ \\
Height & $330 \mathrm{~cm}$ & $189 \mathrm{~cm}$ \\
Vehicle weight & $11,810 \mathrm{~kg}$ & $860 \mathrm{~kg}$ \\
Gross weight & $14,945 \mathrm{~kg}$ & $1,320 \mathrm{~kg}$ \\
Front axle weight & $4,030 \mathrm{~kg}$ & $440 \mathrm{~kg}$ \\
Rear axle weight & $7,780 \mathrm{~kg}$ & $420 \mathrm{~kg}$ \\
\hline
\end{tabular}

(b)

\begin{tabular}{cccccccc}
\hline Items & Model No. & Serial No. & Channel & Axis & Sensitivity & Unit & Location \\
\hline \multirow{3}{*}{ Bus } & SA11ZSC-TI & 5692 & CH1 & $\mathrm{Z}$ & 0.99 & $\mathrm{mV} / \mathrm{ms}^{-2}$ & Center \\
& M353B16 & 106161 & $\mathrm{CH} 2$ & $\mathrm{Z}$ & 1.058 & $\mathrm{mV} / \mathrm{ms}^{-2}$ & Right \\
& M353B12 & 106410 & $\mathrm{CH} 3$ & $\mathrm{Z}$ & 0.483 & $\mathrm{mV} / \mathrm{ms}^{-2}$ & Left \\
\multirow{3}{*}{ Mini vehicle } & \multirow{2}{*}{2422} & 1236 & $\mathrm{CH} 1$ & $\mathrm{Z}$ & 794.0 & $\mathrm{mV} / \mathrm{G}$ & Center \\
& & 1237 & $\mathrm{CH} 2$ & $\mathrm{Z}$ & 795.1 & $\mathrm{mV} / \mathrm{G}$ & Right \\
& & 1238 & $\mathrm{CH} 3$ & $\mathrm{Z}$ & 797.3 & $\mathrm{mV} / \mathrm{G}$ & Left \\
\hline
\end{tabular}

Table 16. Structural specifications of field test bridge (Sakae Bridge).

\begin{tabular}{cc}
\hline Length & $\mathrm{L}=168.29 \mathrm{~m}$ \\
\hline Width & $\mathrm{W}=11.0 \mathrm{~m}$ (two lanes + sidewalks) \\
& $\mathrm{W}=2.5 \mathrm{~m}$ (sidewalks) \\
Span & Eight spans \\
Super structure & Cantilever reinforced concrete (RC) T-girder bridge \\
\hline
\end{tabular}

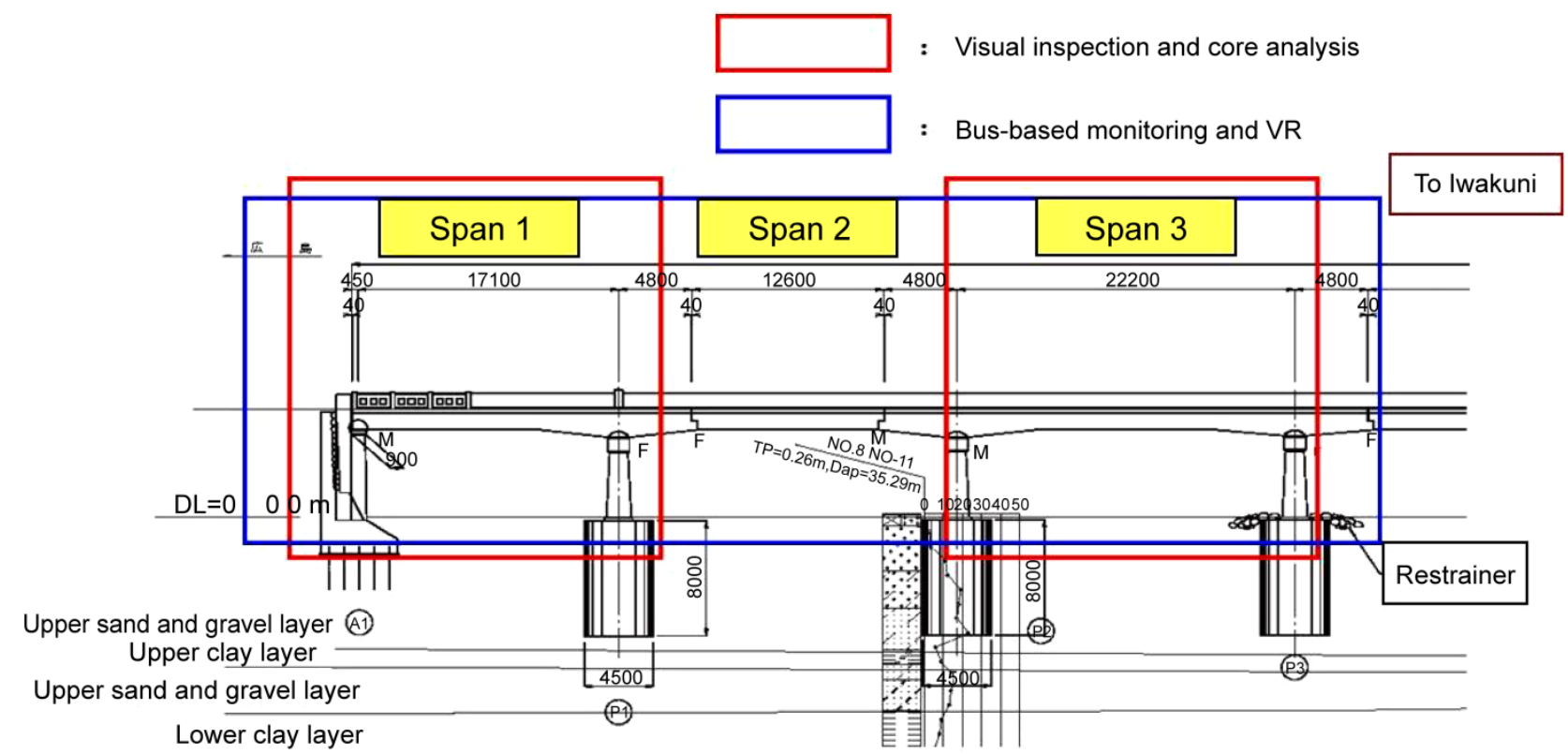

Figure 29. Configuration and dimensions of field test bridge and spans used for field testing. 


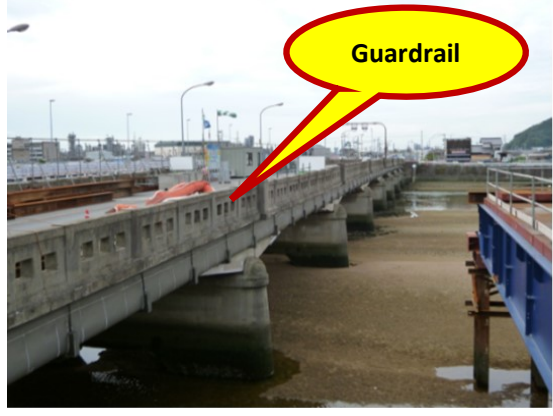

(a)

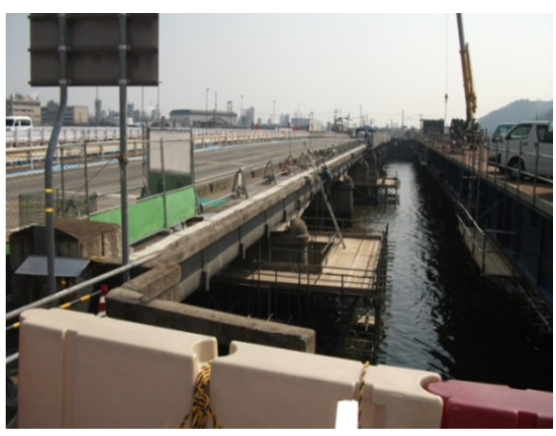

(b)

Figure 30. Bridge before and after guardrail removal. (a) Before guardrail removal; (b) After guardrail removal.

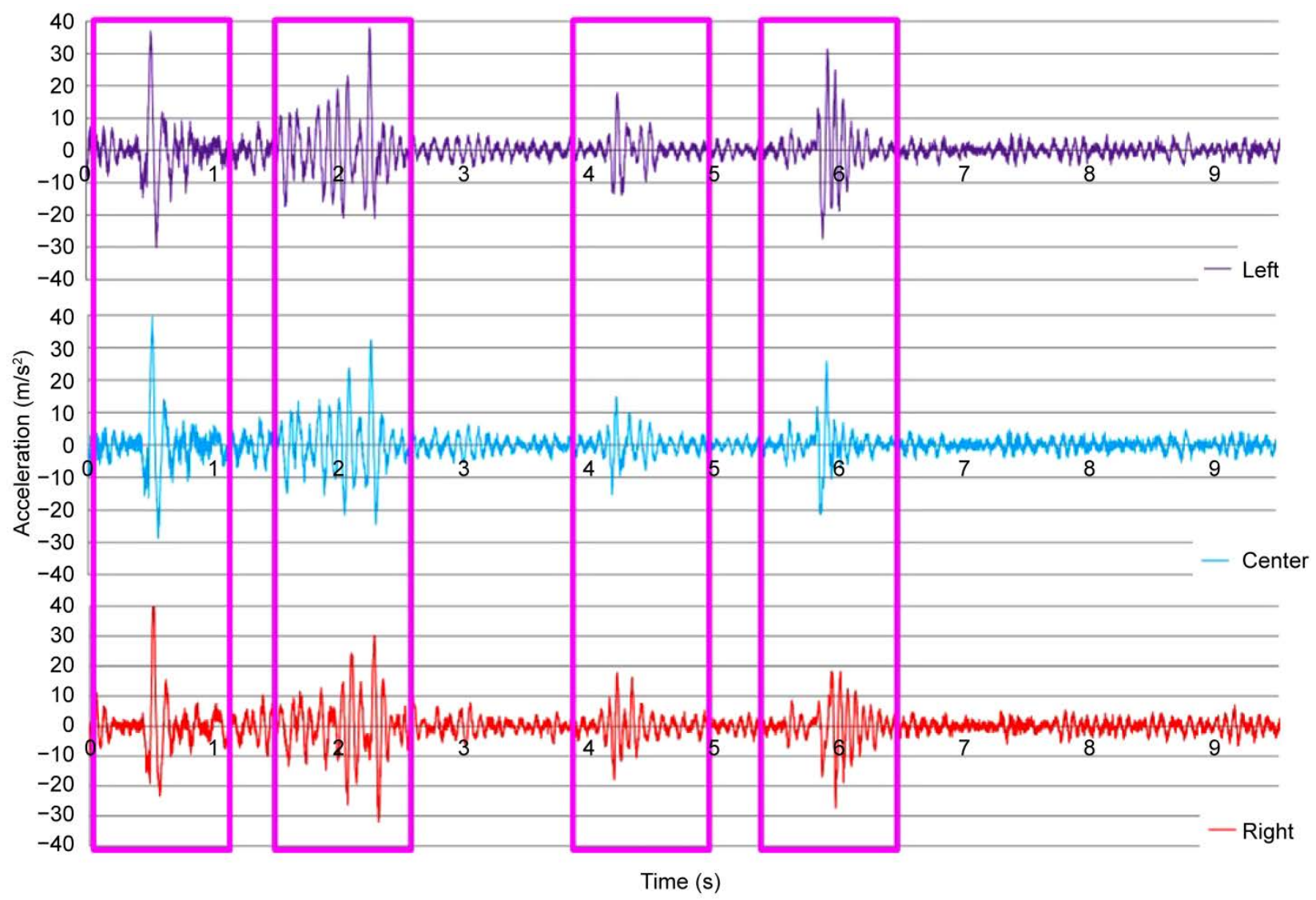

Figure 31. Comparison of acceleration response waveforms obtained from the sightseeing bus.

locations under the rear wheel spring of the sightseeing bus. As Figure 31 indicates, acceleration response can be measured at any sensor location with accuracy good enough to make characteristic deflection calculation possible. From this, it can be said that an acceleration sensor needed by the bus monitoring system to calculate characteristic deflection as a serious damage indicator may be installed at any location under the rear wheel spring.

\subsubsection{Differences in Axle Weight Due to Vehicle Type}

To evaluate the effects of vehicle axle weight on "characteristic deflection", 
"characteristic deflection" was calculated from the acceleration responses of the sightseeing bus and the mini vehicle, which have a gross weight difference of more than $10 \mathrm{tf}$, measured while they were moving. As examples, Figure 32(a) to Figure 32(d) show an under-rear-wheel-spring (center) acceleration response waveform of the sightseeing bus and an under-rear-wheel-spring (right) acceleration response waveform of the mini vehicle, along with acceleration response waveforms of the sightseeing bus and the mini vehicle obtained from the acceleration sensor installed to the bridge when they crossed the bridge. Comparison of Figure 32(a) and Figure 32(b) reveals that the response waveform obtained from the acceleration sensor installed to the mini vehicle tends to be larger, in both the noise portion and the signal portion, than the waveform obtained from the sightseeing bus. The reason for this is thought to be that since the mini vehicle is lighter than the sightseeing bus (smaller axle weight), the former is more easily affected by the roughness of the road surface when in motion so that vertical vibration of the mini vehicle becomes greater. Comparison of Figure 32(c) and Figure 32(d) reveals that the response waveform obtained from the bridge acceleration sensor when the mini vehicle cross the bridge is significantly smaller than the response waveform recorded when the sightseeing bus crossed the bridge. From this, it is presumed that the mini vehicle, whose gross weight is small, is not heavy enough to cause the bridge to vibrate and that external disturbance factors such as road surface roughness prevent the under-spring structure of the vehicle and the bridge from vibrating together (i.e. the similarity between the vehicle and the bridge does not hold). As a result, it is presumed, the

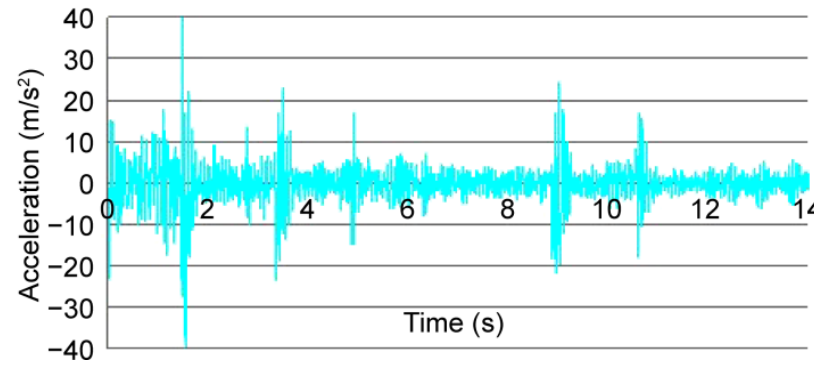

(a)

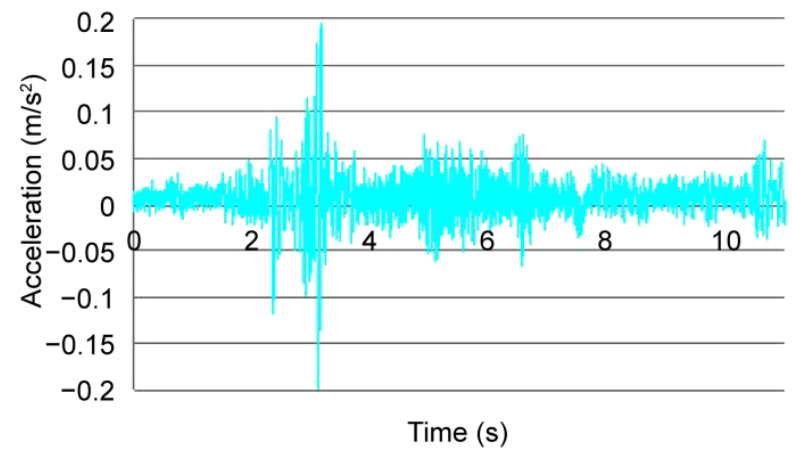

(c)

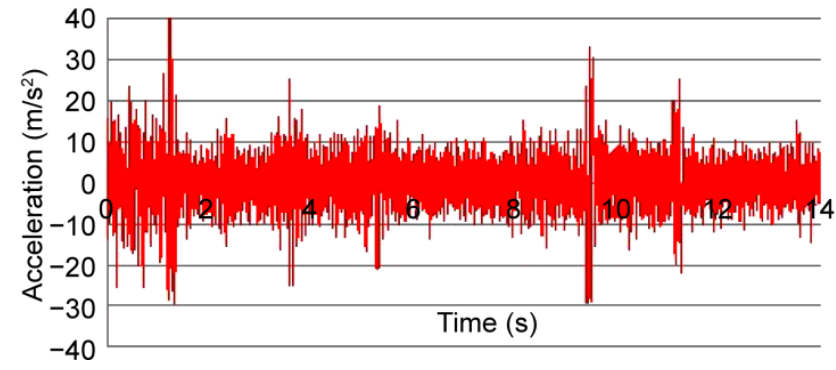

(b)

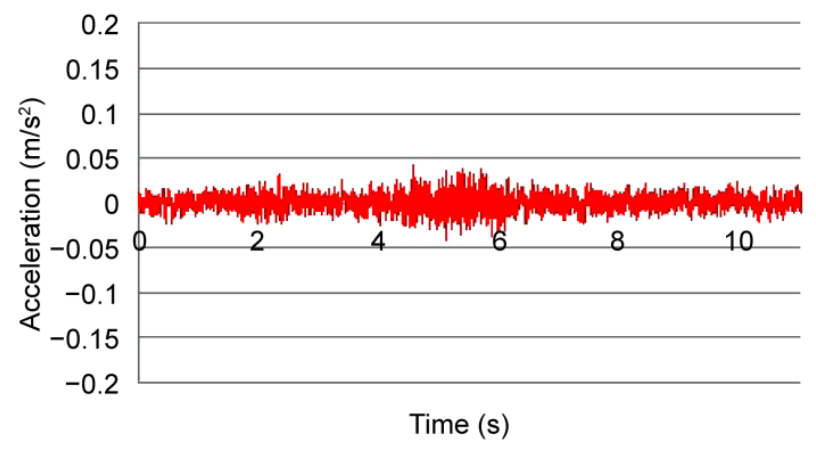

(d)

Figure 32. Effects of vehicle type on vehicle and bridge acceleration response waveforms. (a) Acceleration response waveform of sightseeing bus; (b) Acceleration response waveform of mini vehicle; (c) Bridge acceleration response waveform under moving sightseeing bus loading; (d) Bridge acceleration response waveform under moving mini vehicle loading. 
requirements of the bus monitoring system are not met (i.e. not suitable for the calculation of "characteristic deflection").

\subsubsection{Comparison of Characteristic Deflections before and after Guardrail Removal}

In this section, 15 calculated values of "characteristic deflection" derived from the measurement results obtained at four different times of year by using the sightseeing bus having a gross weight of about $15 \mathrm{tf}$ are used to examine the effects of the artificial damage (guardrail removal) introduced into the bridge ("Sakae Bridge"). As an example, Table 17 and Figure 33 show the "characteristic deflection" calculation results for Span 2 and Span 3 (see Figure 29) of the bridge obtained by using the sightseeing bus (moving at $40 \mathrm{~km} / \mathrm{h}$ ). The concrete guardrail removal was carried out between the second measurement (January 2013) and the third measurement (February 2013). The "characteristic deflection" values obtained before and after the guardrail removal are compared below.

First, turning attention to Span 3 (see Figure 29), we notice that the two characteristic deflection values $(-2.97,-2.89)$ obtained after the guardrail removal are about $10 \%$ larger than the values $(-2.67,-2.61)$ obtained before the guardrail removal. This is thought to be because the decrease in the flexural stiffness of the

Table 17. Calculated values of characteristic deflection before and after guardrail removal (unit: $\mathrm{mm}$ ).

\begin{tabular}{|c|c|c|c|c|c|c|c|c|}
\hline \multirow[t]{3}{*}{ Measurement No. } & \multicolumn{4}{|c|}{ Before guardrail removal } & \multicolumn{4}{|c|}{ After guardrail removal } \\
\hline & \multicolumn{2}{|c|}{$\begin{array}{l}\text { First Measurement } \\
\text { Sept. 11, } 2012\end{array}$} & \multicolumn{2}{|c|}{$\begin{array}{l}\text { Second Measurement } \\
\text { Jan. 10, } 2013\end{array}$} & \multicolumn{2}{|c|}{$\begin{array}{l}\text { Third Measurement } \\
\text { Feb. 12, } 2013\end{array}$} & \multicolumn{2}{|c|}{$\begin{array}{l}\text { Fourth Measurement } \\
\text { March 5, } 2013\end{array}$} \\
\hline & Span 2 & Span 3 & Span 2 & Span 3 & Span 2 & Span 3 & Span 2 & Span 3 \\
\hline 1 & -3.347 & -2.668 & -2.727 & -2.477 & -2.130 & -2.264 & -1.510 & -2.418 \\
\hline 2 & -2.723 & -2.083 & -1.854 & -3.262 & -2.081 & -2.468 & -2.194 & -2.951 \\
\hline 3 & -1.702 & -3.326 & -2.999 & -2.003 & -2.158 & -1.886 & -3.042 & -3.719 \\
\hline 4 & -2.341 & -2.237 & -3.068 & -2.699 & -2.652 & -4.045 & -1.867 & -3.100 \\
\hline 5 & -1.976 & -1.104 & -2.524 & -2.866 & -3.289 & -3.241 & -1.922 & -2.482 \\
\hline 6 & -1.704 & -1.780 & -4.348 & -2.412 & -1.675 & -2.650 & -1.660 & -2.669 \\
\hline 7 & -2.885 & -3.595 & -0.854 & -2.739 & -3.421 & -2.923 & -2.060 & -2.544 \\
\hline 8 & -2.636 & -2.449 & -2.179 & -2.781 & -3.197 & -3.454 & -2.591 & -1.790 \\
\hline 9 & -1.359 & -2.607 & -1.510 & -2.019 & -2.769 & -4.074 & -2.562 & -1.941 \\
\hline 10 & -1.794 & -1.507 & -2.119 & -2.323 & -2.725 & -2.936 & -2.466 & -2.057 \\
\hline 11 & -1.618 & -3.852 & -1.235 & -2.014 & -1.961 & -2.372 & -2.315 & -3.023 \\
\hline 12 & -2.647 & -3.419 & -3.159 & -3.124 & -2.554 & -3.317 & -1.639 & -.872 \\
\hline 13 & -2.819 & -3.075 & -2.720 & -2.710 & -2.522 & -2.477 & -1.604 & -3.404 \\
\hline 14 & -1.568 & -3.043 & -1.409 & -3.183 & -2.334 & -3.094 & -2.398 & -3.583 \\
\hline 15 & -3.421 & -3.321 & -1.636 & -2.593 & -3.606 & -3.337 & -3.815 & -4.857 \\
\hline Average & -2.303 & -2.671 & -2.289 & -2.614 & -2.605 & -2.969 & -2.243 & -2.894 \\
\hline
\end{tabular}




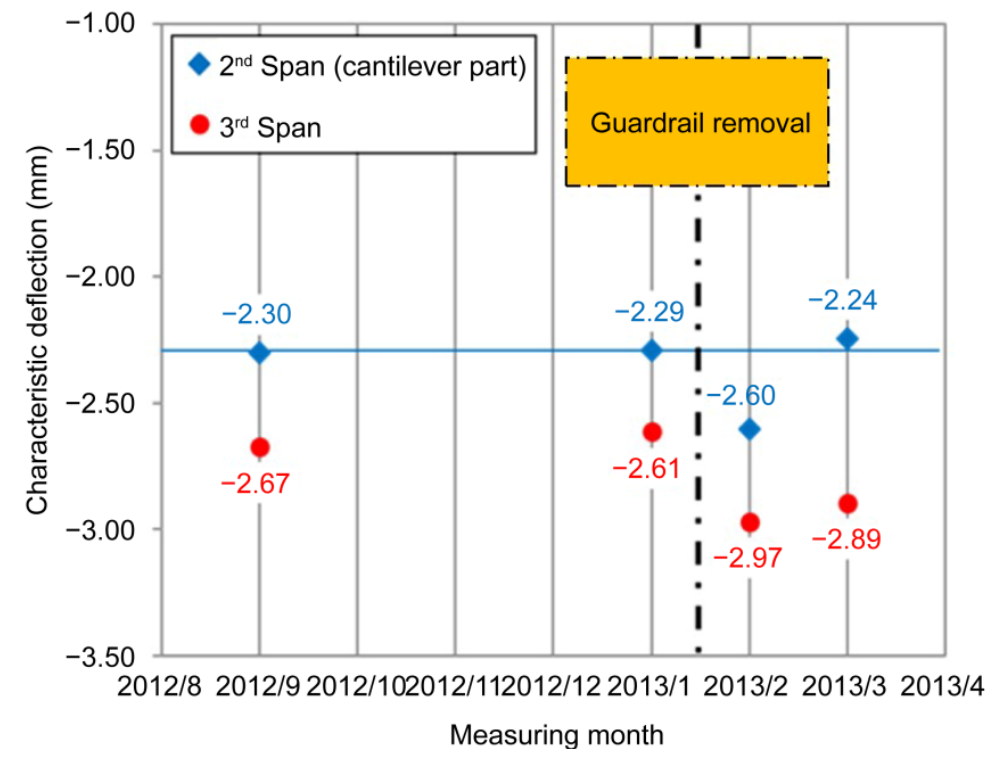

Figure 33. Changes in characteristic deflection due to guardrail removal.

bridge resulted in an increase in "characteristic deflection". In Span 2, which includes a cantilever structure, however, the characteristic deflection value $(-2.24)$ obtained after the fourth measurement is slightly smaller than the value obtained before the guardrail removal. Since Span 2 includes a cantilever structure, it is thought likely that the cantilever structure somehow influenced the "characteristic deflection". When calculating characteristic deflection of a bridge with a cantilever structure, therefore, it is necessary to perform a simulation analysis and compare the calculated values with the analytical results.

\subsection{Analytical Study}

This section deals with a model-based simulation analysis of the bridge measurement results obtained from the bus monitoring system performed to analytically identify the bridge behavior before and after the guardrail removal.

In the analysis, a static FEM analysis [30] was conducted of the simple girder structure excluding Span 2 on the Hiroshima side to calculate the maximum deflection ratio and evaluate the influence of the bridge guardrails on girder stiffness. The analysis, by using the three-dimensional finite analysis method, calculates and compares the midspan deformation in the cases where a midspan vertical downward unit concentrated load $(1 \mathrm{kN})$ is applied. Static analyses were conducted of the four cases listed below:

Case 1: With guardrails, no damage,

Case 2: Without guardrails, no damage,

Case 3: With guardrails, damaged,

Case 4: Without guardrails, damaged,

The analysis used MIDAS-GEN (MIDAS-IT Co.).

\subsubsection{Analysis Model and Analysis Conditions}

Figure 34 shows how the analysis model used looks. Figure 35 shows an 


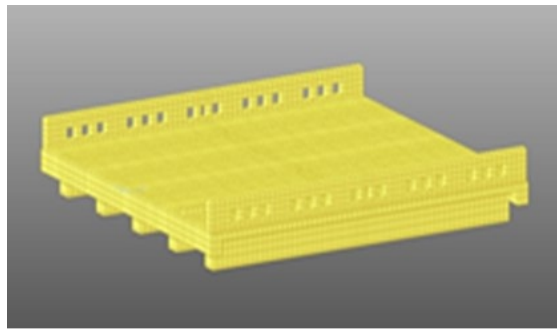

(a)

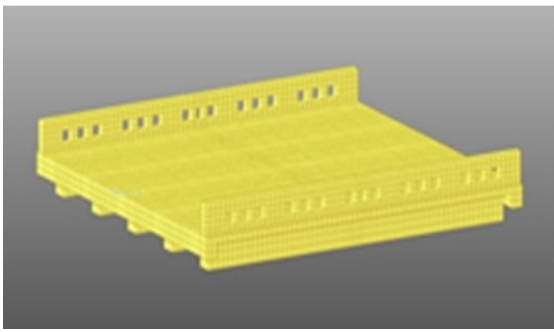

(c)

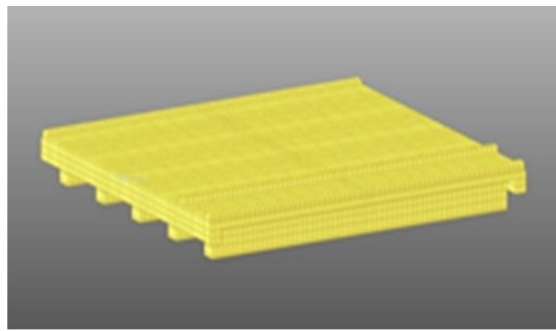

(b)

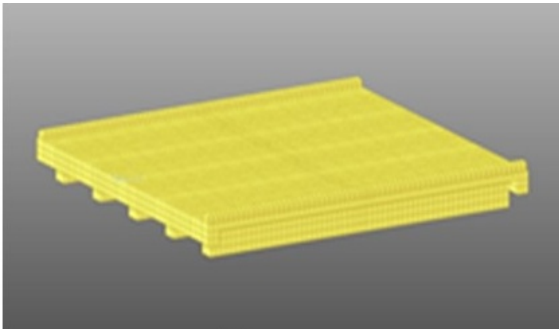

(d)

Figure 34. Model used for analysis. (a) Case 1; (b) Case 2; (c) Case 3; (d) Case 4.
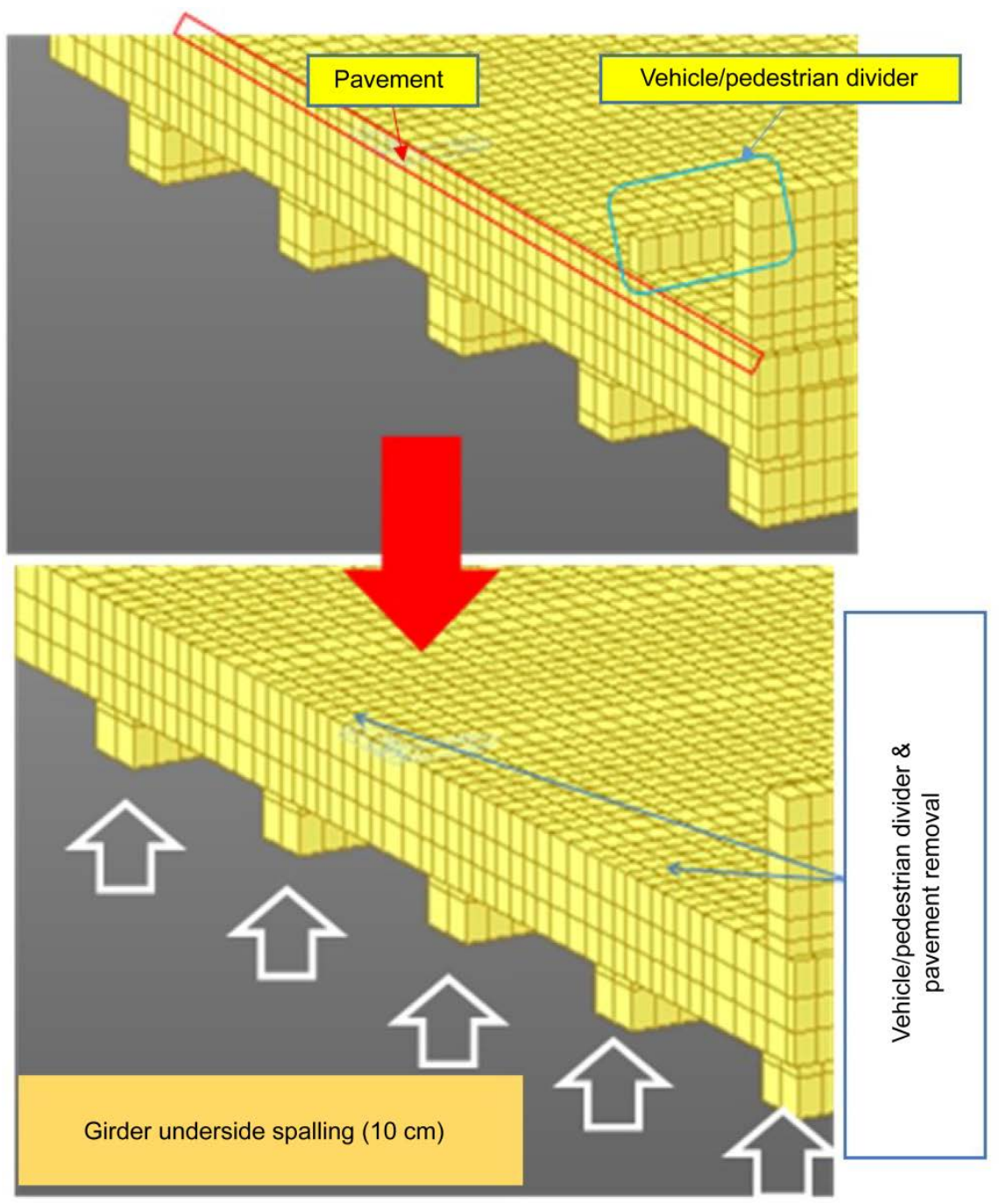

Figure 35. Assumed damage. 
enlarged view of an assumed damage region in the "damaged" case shown in Figure 34. The static analysis conditions are shown in Table 18. An eigenvalue analysis showed that the first mode natural frequency is $16 \mathrm{~Hz}$. The first mode frequency determined from theoretical solutions and other study findings [31] [32] is also $16 \mathrm{~Hz}$. It is therefore thought that the model mentioned above is suitable for use in this study.

\subsubsection{Comparison between Analytical Results and Characteristic Deflection Values}

Table 19 shows the obtained analytical results. The change ratio in the "with guardrails and no damage" case under the assumed conditions is 1.04, indicating that the change amounts only to about $4 \%$. The change ratio in the case where general delamination of the underside of the girder is assumed is about 1.05, indicating a change of about $5 \%$. The geometrical moment of inertia was also calculated in both the "with guardrails" and "without guardrails" cases. The second moments of area thus obtained are shown in Table 20. As shown, the geometrical moment of inertia shows a decrease of about $40 \%$ as a result of the guardrail removal. This translates to a deflection ratio of 1.64 .

Table 18. Analysis conditions.

\begin{tabular}{cl}
\hline \multicolumn{1}{c}{ Items } & \multicolumn{1}{c}{ Conditions } \\
\hline Analysis method & Static elasticity analysis using 3D solid elements \\
& JIS (RC) Fc24 or equivalent \\
& Modulus of elasticity: 2.2668E7 \\
Material constants & Poisson's ratio: 0.2 \\
& Unit weight: 24.0 \\
& Midspan vertical downward unit concentrated load (1 kN) \\
Boundary conditions & Simple beam; restrained at bottom of girder end \\
\hline
\end{tabular}

Table 19. Analysis results (midspan deflection).

\begin{tabular}{ccc}
\hline Case & Deflection $(\neq$ characteristic deflection) & Ratio to "with guardrail” case \\
\hline 1 & $-2.89 \times 10^{-6} \mathrm{~m}$ & \\
2 & $-3.00 \times 10^{-6} \mathrm{~m}$ & $1.04(4 \%$ increase $)$ \\
3 & $-3.29 \times 10^{-6} \mathrm{~m}$ & $1.05(5 \%$ increase $)$ \\
4 & $-3.47 \times 10^{-6} \mathrm{~m}$ & \\
\hline
\end{tabular}

Table 20. Geometrical moment of inertia calculation results.

\begin{tabular}{cccc}
\hline Items & With guardrails & Without guardrails & Decrease ratio \\
\hline Cross-sectional area $\left(\mathrm{m}^{2}\right)$ & 6650 & 5948 & $10.5 \%$ \\
Neutral axis location $(\mathrm{m})$ & 0.974 & 0.877 & \\
Geometrical moment of inertia $\left(\mathrm{m}^{4}\right)$ & 1.612 & 0.980 & $39.2 \%$ \\
\hline
\end{tabular}


The results of the 3D FEM analysis mentioned earlier show that the change ratio ranges from 1.04 to 1.05 , and the effects of the guardrail removal are very small compared with the values obtained from the geometrical moment of inertia. This is thought to indicate that stress transmission paths extend two-dimensionally under the midspan loading due to the moving bus. This does not agree with the result of stiffness evaluation based on the assumption, for cross-sectional calculation, of Navier's hypothesis (of plane sections remaining plane) and bending in the bridge axis direction.

Comparison with characteristic deflection reveals that the calculated values of "characteristic deflection" for Span 2 show decreases after the guardrail removal.

\subsection{Summary}

According to the "characteristic deflection" calculation results for Span 3, the "characteristic deflection" values obtained after the guardrail removal (average: $2.93 \mathrm{~mm}$ ) indicate a decrease of about $10 \%$ compared with the characteristic deflection values (average: $2.64 \mathrm{~mm}$ ) obtained before the guardrail removal. In view of the fact that the change ratio obtained through the static analysis in the case assuming general delamination of the underside of the girder was about $5 \%$, it can be said that the "characteristic deflection" is reasonably sensitive to the guardrail removal. For Span 2, which includes a cantilever structure, however, the "characteristic deflection" after the guardrail removal showed a slight increase from the value obtained before the guardrail removal. This result is not consistent with the results of 3D FEM analysis, either. One likely reason for this is that the model used for the analysis consists of a simple span without a cantilever structure, while the calculated values of "characteristic deflection" have been influenced by the cantilever structure. Changes in measurement results due to seasonal factors are possible, but that seems unlikely in view of the results for Span 3. Span 2 requires further study, and it is also necessary to examine other factors such as the possible influence of the type of bridge structure on "characteristic deflection".

These results have shown, at least at this stage of study in which the usefulness and validity of "characteristic deflection" as an evaluation indicator for use by the bus monitoring system is being verified, that the method of using a threedimensional model for comparison and evaluation is useful when measuring a bridge in which stress transmission paths extend two-dimensionally.

\section{Conclusions}

Short and medium span bridges in Ube-city have been monitored over a long period of time by using the bus monitoring system operated in the city's bus network. This paper has presented the results of long-term observation of "characteristic deflection" used as an evaluation indicator and described a newly developed characteristic deflection observation method. Damage detection performance of the "characteristic deflection" has also been verified systematically by introducing artificial damage (guardrail removal) into a decommissioned bridge 
that was in the process of demolition. Main findings of this study are summarized below:

1) For the purpose of long-term monitoring, a field test was conducted over a period of four years by using an in-service fixed-route bus. The observation of changes in "characteristic deflection" has not revealed any significant changes, and measurement needs to be continued for further observation.

2) On the basis of previous study results, the moving average method has been used to reduce fluctuations of "characteristic deflection" measurements in the bus monitoring system. The moving average method used in this study is the simple moving average method, which calculates simple averages for different data sections without weighting data. The observation of changes in "characteristic deflection" by the simple moving average method has not revealed any significant sharp changes. It can be concluded, at least at this stage, that none of the observed bridges has been seriously damaged.

3) The method of observing changes in "characteristic deflection" by the simple moving average method has been shown to have some problems. One of them is excessive smoothing out of data, and another is that the indication of serious damage by calculation results lags considerably behind real-time data. In order to solve these problems, the simple moving average method was evaluated through comparison with other moving average methods (weighted moving average method, exponential moving average method). Anomalous values were given to the moving average curves of "characteristic deflection" obtained by each moving average method, and the number of inputs needed to reach the deterioration criterion level was compared. As a result, it has been shown that the deterioration criterion level is reached earlier when weighted moving averages or exponential moving averages are used than when simple moving averages are used.

4) The comparison of the number of inputs needed to reach the deterioration criterion level raised concern about a possible delay in detecting an abrupt change in the condition of the bridge being monitored because a certain number of inputs were needed, regardless of the type of moving averages used, until the deterioration criterion level was reached. Attention was turned, therefore, to the slope of the moving average curve in making comparisons. As a result, by comparing the case where an abrupt change (assumed damage) is given and the case based on the actual changes observed thus far, it was concluded that differences indicated by simple moving averages are too small to detect an abrupt change in the bridge condition. In contrast, weighted moving averages and exponential moving averages showed certain degrees of difference. It has therefore been decided to use weighted moving averages, instead of simple moving averages, for "characteristic deflection" monitoring because weighted moving averages tend to show greater differences.

5) On the basis of the results mentioned above, as a next step it is necessary, judging from the slope data (slope of the weighted moving average curve: approx. -0.7 ) obtained in this study, to develop a new set of deterioration evalua- 
tion criteria, without sticking to the current deterioration evaluation criteria.

6) In the field test conducted by using a bridge that was decommissioned and was undergoing demolition, the influence (sensitivity) of artificial damage (guardrail removal) on "characteristic deflection" was evaluated. The results obtained for Span 3 of the bridge confirmed that "characteristic deflection" is reasonably sensitive to a decrease in the flexural stiffness of the entire bridge. In the case of Span 2, which includes a cantilever structure, however, the "characteristic deflection" before the guardrail removal was greater than the "characteristic deflection" after the guardrail removal. This result is not consistent with the 3D FEM analysis results, either. It is likely that the calculated values of "characteristic deflection" have been influenced by the cantilever structure. Further study is needed, therefore, particularly on the influence of the type of bridge structure on "characteristic deflection".

7) At present, the usefulness and validity of "characteristic deflection" as an evaluation indicator are being evaluated and verified. It can be concluded, at least at this stage, that the method of using a three-dimensional model for comparison and evaluation is useful when measuring a bridge in which stress transmission paths extend two-dimensionally.

Finally, the long-term field test of the bus monitoring system has produced useful results associated with the practical application of the system although a number of problems still remain to be solved. Important challenges for the bus monitoring system include the automation and rationalization of measurement. The structural health evaluation of bridges can be made simpler and more efficient by analyzing and solving the problems identified as a result of this study.

\section{Acknowledgements}

The authors would like to express our sincere thanks to Dr. Emoto, H. of Kouzoubutsu Clinic Co., Ltd. for his great support on this research work, and also to the Traffic Division of Ube-city's Transportation Bureau for the generous cooperation in connection with the long-term field test conducted for the purposes of this study including bus sensor installation and bus operation arrangements.

\section{References}

[1] Ministry of Land, Infrastructure, Transport and Tourism (2013) White Paper on Land, Infrastructure, Transport and Tourism in Japan. (In Japanese)

[2] Furuta, H., Kayano, M. and Watanabe, M. (2007) Present State and Future Outlook of Bridge Maintenance and Bridge Management Systems. Doboku Gakkai Ronbunshu F, 63, 287-294. https://doi.org/10.2208/jscejf.63.287

[3] JSCE Committee on Formulation of Future Vision (2014) A 100-Year Vision of Society and Civil Engineering: Opening up Every Possible Opportunity to Build a Sustainable Society. Japan Society of Civil Engineers, Tokyo, Japan.

[4] Road Management Division, Public Works and Construction Department, Yamaguchi Prefectural Government (2014) Yamaguchi Prefecture Bridge Life Extension and Rehabilitation Plan. March 2014 version, Yamaguchi, Japan. (In Japanese)

[5] Kim, C., and Kawatani, M. (1992) Bridge Health Evaluation using Bridge Vibration 
Data Obtained by Use of a Single Passing Vehicle. Journal of Construction Steel, 38A, 781-788.

[6] Lin, C.W. and Yang, Y.B. (2005) Use of a Passing Vehicle to Scan the Fundamental Bridge Frequencies. An Experimental Verification Engineering Structures, 27, 1865 1878. https://doi.org/10.1016/j.engstruct.2005.06.016

[7] Oshima, Y., Yamamoto, R., and Sugiura, K. (2011) Bridge Damage Identification Method Based on Displacement Estimated from Vehicle Response. Proceedings of the 57 th Symposium on Structural Engineering, 57A, 646-654.

[8] Miyamoto, A. and Isoda, S. (2012) Sensitivity Analysis of Mechanical Behaviors for Bridge Damage Assessment. Structural Engineering and Mechanics, 41, 539-558. https://doi.org/10.12989/sem.2012.41.4.539

[9] Miyamoto, A. and Yabe, A. (2012) Development of Practical Health Monitoring System for Short and Medium-Span Bridges Based on Vibration Responses of City Bus. Journal of Civil Structural Health Monitoring, 2, 47-63. https://doi.org/10.1007/s13349-012-0017-0

[10] Yabe, A., Miyamoto, A. and Nagata, M. (2015) Verification Tests for Practical Application of a Health Monitoring System for Short- and Medium-Span Bridges Based on Public Bus Vibrations. Journal of Civil Structural Health Monitoring, ISHMII, 5, 67-95. https://doi.org/10.1007/s13349-014-0094-3

[11] Yabe, A. (2006) Development of a Method of Analyzing Dynamic Moving BodyStructure Interaction Using the Substructure Method. Proceedings of the 61 st Annual Conference of the Japan Society of Civil Engineers, Vol. 1, 845-846.

[12] Tanaka, H., Miyamoto, A., Emoto, H., and Yabe, A. (2014) Bus-Based Structural Health Monitoring System for Smaller Bridges: Long-Term Field Test and Damage Detection. Doboku Gakkai Ronbunshu F3 (Civil Engineering Informatics), 70, I_193-I_206.

[13] Yabe, A., Miyamoto, A., Isoda, S., and Tani N. (2013) Development of Techniques for Short/Medium-Span Bridge Monitoring System Using In-Service Fixed-Route Bus. Doboku GakkaiRonbunshu, F4 (Construction Management), 69, 102-120. https://doi.org/10.2208/jscejcm.69.102

[14] Kyoryo Shindo Kenkyukai (1993) Measurement and Analysis of Bridge Vibration. GihodoShuppan, October 1993 Version. (In Japanese).

[15] Japan Society of Civil Engineers (JSCE) (2000) Guidelines for Bridge Vibration Monitoring. Structural Engineering Series, 10, 110-119.

[16] Japan Society of Mechanical Engineers (JSME) (1987) Computer Analysis in Mechanical Engineering. Corona Publishing, Tokyo.

[17] Ube City Transportation Bureau (UTB) (2013) Ube City Municipal Bus Route Map (as of October 1, 2013). http://www.ubebus.jp/rosenzu2013.pdf

[18] Ube City (2013) Bridge Life Extension and Rehabilitation Plan. December 2013 version.

http://www.city.ube.yamaguchi.jp/machizukuri/dourokasen/dourokasen/document s/kyouryou-h23_2.pdf

[19] Fuji Ceramics Corporation (2012) Triaxial Accelerometer Specifications. January 24 Published Version 2012. http://www.fujicera.co.jp/product/j/04/07.html

[20] Yabe, A. (2006) Development of Moving Body-Structure Interaction Analysis Technique Using Substructure Method. Proceedings of the 61 st Annual Conference of the Japan Society of Civil Engineers, Tokyo, September 2006, 845-846.

[21] Yan, Z.-H., Miyamoto, A. and Jiang, Z.-W. (2009) Frequency Slice Wavelet Transform for Transient Vibration Response Analysis. Mechanical Systems and Signal 
Processing, 23, 1474-1489. https://doi.org/10.1016/j.ymssp.2009.01.008

[22] Yan, Z.-H., Miyamoto, A., Jiang, Z.-W. and Liu, X.-L. (2010) An Overall Theoretical Description of Frequency Slice Wavelet Transform. Mechanical Systems and Signal Processing, 24, 325-572. https://doi.org/10.1016/j.ymssp.2009.07.002

[23] Yabe, A., Isoda, S., Nagata, M., and Miyamoto, A. (2012) Field Test of Short/ Medium-Span Bridge Monitoring System Using In-Service Fixed-Route Bus. Proceedings of the 67 th Annual Conference of the Japan Society of Civil Engineers, Tokyo, September 2012, VI-232.

[24] National Institute for Land and Infrastructure Management and Japan Prestressed Concrete Contractors Association (2010) Joint Study on Refinement of Structural Health Evaluation of Prestressed Concrete Road Bridges, Chapter 4. Technical Note of NILIM No. 613, 99-105. (In Japanese)

[25] Yabe, A., Miyamoto, A., Isoda, S. and Tani N. (2013) Development of Techniques for Short/Medium-Span Bridge Monitoring System Using In-Service Fixed-Route Bus. Doboku Gakkai Ronbunshu F4 (Construction Management), 69, 102-120.

[26] Nosaka, K. and Masuda, K. (2010) A New Approach to Moving Average Interpretation: Predicting Market Conditions from Six Positions: Identifying the Right Timing of Long-Term/Short-Term Investment. Kanki Publishing, Tokyo, Japan.

[27] Kanatani, K. (2005) Mathematical Optimization Made Easy. Kyoritsu Shuppan, Tokyo, Japan.

[28] Ishimura, S. (1989) Statistical Analysis Explained. Tokyo Tosho Publisher, Tokyo, Japan.

[29] Miyamoto, A., Emoto, H., Takahashi, J. and Yabe, A. (2015) Structural Health Evaluation and Remaining Life Estimation of a Decommissioned Bridge Based on Site Investigation: Sakae Bridge Investigation Results. Infrastructure Management Series No. 22, The Research Center for Environmental Safety, Yamaguchi University, Ube, Japan, 1-104.

[30] Yabe, A. (2011) Fundamental Study on Development of Bridge Anomaly Detection System Using In-Service Fixed-Route Bus. Doctoral Dissertation, Yamaguchi University, Ube, Japan.

[31] Omi, H., Mori, S. and Twayan, R.P. (2013) Detectability of Natural Frequency of Concrete Girder Bridge by Vehicle Loading and Hammering. Proceedings of the Annual Conference of JSCE Shikoku Branch, Takamatsu, June 2013, I-4.

[32] Tamada, K. (2013) Experimental Study on Damage and Dynamic Characteristics of Bridges Managed by Local Governments. Japan Institute of Countryology and Engineering, Proceedings of the 13 th JICE Annual Presentation of Subsidized Research, Tokyo, June 2013, I-5. 
Submit or recommend next manuscript to SCIRP and we will provide best service for you:

Accepting pre-submission inquiries through Email, Facebook, LinkedIn, Twitter, etc. A wide selection of journals (inclusive of 9 subjects, more than 200 journals)

Providing 24-hour high-quality service

User-friendly online submission system

Fair and swift peer-review system

Efficient typesetting and proofreading procedure

Display of the result of downloads and visits, as well as the number of cited articles Maximum dissemination of your research work

Submit your manuscript at: http://papersubmission.scirp.org/

Or contact eng@scirp.org 